\title{
Effectiveness of Community-Delivered Parent-Child Interaction Therapy Compared to Treatment as Usual
}

Jocelyn Stokes

West Virginia University

Follow this and additional works at: https://researchrepository.wvu.edu/etd

\section{Recommended Citation}

Stokes, Jocelyn, "Effectiveness of Community-Delivered Parent-Child Interaction Therapy Compared to Treatment as Usual" (2014). Graduate Theses, Dissertations, and Problem Reports. 197.

https://researchrepository.wvu.edu/etd/197

This Dissertation is protected by copyright and/or related rights. It has been brought to you by the The Research Repository @ WVU with permission from the rights-holder(s). You are free to use this Dissertation in any way that is permitted by the copyright and related rights legislation that applies to your use. For other uses you must obtain permission from the rights-holder(s) directly, unless additional rights are indicated by a Creative Commons license in the record and/ or on the work itself. This Dissertation has been accepted for inclusion in WVU Graduate Theses, Dissertations, and Problem Reports collection by an authorized administrator of The Research Repository @ WVU.

For more information, please contact researchrepository@mail.wvu.edu. 
Effectiveness of Community-Delivered Parent-Child Interaction Therapy Compared to Treatment as Usual

Jocelyn Stokes, M. S., BCBA

Dissertation submitted to the Eberly College of Arts and Sciences

at West Virginia University

in partial fulfillment of the requirements for the degree of

Doctor of Philosophy in

Psychology

\author{
Cheryl B McNeil, Ph. D., Chair \\ Christina L. Duncan, Ph. D. \\ Elisa Krackow, Ph. D. \\ Katherine Karraker, Ph. D. \\ Dan Hursh, Ph. D. \\ Department of Psychology
}

\title{
Morgantown, West Virginia 2014
}

Keywords: Parent-Child Interaction Therapy; effectiveness; community intervention; disruptive behavior

Copyright 2014 Jocelyn Stokes 


\begin{abstract}
Effectiveness of Community-Delivered Parent-Child Interaction Therapy Compared to Treatment as Usual
\end{abstract}

Jocelyn Stokes, M. S., BCBA

Parent-Child Interaction Therapy (PCIT) is a "probably efficacious" treatment for children with disruptive behavior disorders (Eyberg, Nelson, \& Boggs, 2008). However, many of the characteristics of the efficacy studies (e.g., therapy conducted in university-based clinics, graduate student therapists, supervision and feedback on integrity to the model, recruited families, homogeneous samples) supporting PCIT may limit the generalization of the results to the community setting where most families receive treatment (e.g., Weisz, Jensen-Doss, \& Hawley, 2006). Few studies of PCIT have examined the effectiveness of PCIT implemented by community therapists, and these studies have many methodological limitations including no reliability of observational data, no examination of treatment integrity, and no comparison group. Almost no studies have compared PCIT to usual care (UC; for exception, see McCabe \& Yeh, 2009). The current study was a prospective examination of the short-term treatment outcomes for young children with disruptive behavior who received PCIT delivered by community therapists compared to UC.

Thirty caregiver-child dyads participated in the study. Most children were male (57\%) and Caucasian (79\%) with an average age of 5 years. Most children had a disruptive behavior disorder diagnosis $(63 \%)$ or Autism Spectrum Disorder $(23 \%)$. Most caregivers were female (97\%), biological parents (93\%) with an average age of 29 years. Families received services from seventeen therapists at five agencies. All therapists were Caucasian females with master's degrees. Therapists had an average of 6 years 6 months experience providing therapy. PCIT therapists had completed zero to four PCIT cases prior to enrolling families in the study.

Two assessments were conducted in the families' homes about seven and a half months apart. Families in the PCIT group had not yet received more than two coaching sessions of Child-Directed Interaction. Families in the UC group had not yet received services for more than six weeks. Parent-reported child behavior was examined using the Child Behavior Checklist (CBCL; Achenbach \& Rescorla, 2000; 2001) and the Eyberg Child Behavior Inventory (ECBI; Eyberg \& Pincus, 1999). Parent and child behavior during 15 minutes of observation was coded by undergraduate research assistants using the Dyadic Parent-Child Interaction Coding System (DPICS; Eyberg, Nelson, Duke, \& Boggs, 2005), and the reliability of the observational coding was examined. Parenting stress was examined using the Parenting Stress Index (Abidin, 1995). Therapists provided descriptions of treatment, and their reports were compared to the PCIT manual (Eyberg \& Funderburk, 2011) to estimate treatment integrity. Parental satisfaction with treatment was examined quantitatively and qualitatively. Therapists also provided a qualitative report of acceptability of PCIT.

Families in the PCIT group received a median of 10 sessions of PCIT and a median of 17 hours of total therapy. Thirty three percent of families in PCIT had completed treatment or were still participating in PCIT at the second assessment. UC families received approximately 162 median hours of therapeutic service in the home. Sixty percent of families in UC had completed therapy or were receiving the same treatment modality at the second assessment. Results indicated that PCIT was delivered with about $74 \%$ reported treatment integrity. PCIT significantly differed from UC in all aspects of treatment delivery examined in the current study (e.g., provision and review of homework, data collection of observed parent and child behavior). Most analyses showed no statistically significant differences between PCIT and UC on standardized outcome measures; however, the lack of significant differences may be misleading due to the small sample size and increased likelihood of a Type II error. Effect sizes for measures 
of child behavior were .64 to .93 , indicating medium to large effects, in the PCIT group, and .25 to .39 , indicating small to medium effects, in the UC group. No differences were found with regard to caregiver satisfaction.

Regarding treatment outcomes for child behavior, results indicate that PCIT was at least equivalent to UC in effectiveness and may have had a greater effect on child behavior based on outcomes measured in the current study. PCIT shows promise as a cost-effective treatment for young children with disruptive behavior, as it may produce larger effects with fewer service hours. Regarding the dissemination of PCIT, results indicate that few therapists trained in PCIT continue to actively implement it for a variety of reasons, including administrative responsibilities and insurance barriers. However, those who continue to use PCIT achieve meaningful improvements in child behavior and find it to be a preferred model for treating young children with disruptive behavior.

Attrition within the study limited the sample size and statistical power to find significant differences between the groups. Conclusions from the current study are also limited because participants were not randomized to condition. More research is needed to examine the effectiveness of PCIT in the community, including its cost-effectiveness compared to UC and identification of the critical components of PCIT. Also, effective training of community therapists in PCIT and strategies to decrease attrition from treatment are areas in need of further research. 


\section{Acknowledgements}

This dissertation research could not have been completed without the administrators and therapists who supported this project. I am grateful to the administrators who allowed me to recruit therapists and participants from their sites. I give special thanks to Rick Murray, MSW, BCBA, Bethany Connors, LCSW, Amy Fisher, LCSW, Karen Barkley, LCSW, and Amanda Newhouse, LCSW, because I would have had no participants without their support in connecting me with therapists. I have the utmost respect for the therapists who bravely allowed me to examine their clients' treatment outcomes. Also, I appreciate that the participating therapists generously donated their time to tell families about the study and complete questionnaires to describe the families and treatment without financial compensation. I would like to acknowledge my "favorite outpatient therapist," who shall remain unnamed to protect her confidentiality, Amy Fisher, LCSW, and Karen Barkley, LCSW, for their willingness to answer multiple follow up questions about treatment delivery and completion. The willingness of these administrators and therapists to participate reveals their dedication to providing high quality services to children and families. I would like to thank the families who allowed a previously unknown person to come into their home to collect data. I appreciate that they completed lengthy questionnaires and let me videotape in their home. These families have contributed to a greater understanding of treatment outcomes that may benefit families seeking therapeutic services in the future.

I thank Cheryl McNeil, Ph. D., and the members of her lab in the Department of Psychology at West Virginia University for their support and assistance with the research project. I am grateful for Dr. McNeil's guidance, time, and emotional support throughout my graduate career. I appreciate her willingness to take a risk in allowing a student to conduct an effectiveness study. Special thanks to Nancy Wallace, Rachel DeRoos, Kelsey Sommersville, Meggie Swisher, Taylor Louk, Lindsey Ives, and Jacklyn Stankus who gave many hours and some Saturdays to assist with home visits and/ or coding of observational data. I would like to acknowledge the statistical guidance of Aaron Metzger, Ph. D. I appreciate the contributions of 
my committee members in strengthening this dissertation, and I thank the Department of Psychology and Eberly College of Arts and Sciences at West Virginia University for the Student Dissertation Award that assisted with financial expenses associated with this research.

Lastly, I would like to thank my friends and family who provided immeasurable emotional support throughout my graduate career and dissertation. They provided much needed perspective and distraction. I also thank my prior supervisors and coworkers, including Demy Kamboukos, Ph. D., who helped me to pursue further training in psychology. I feel blessed to have such intelligent and caring people in my life. 


\section{Table of Contents}

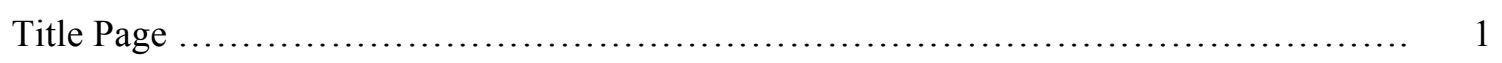

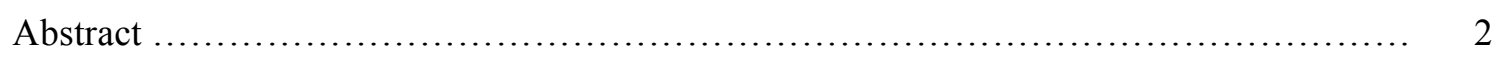

Acknowledgements ....................................................... 4

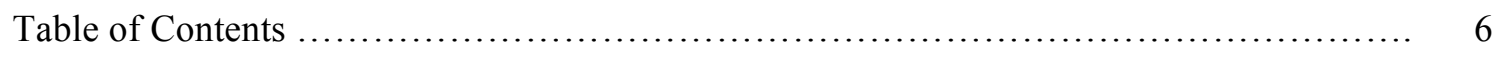

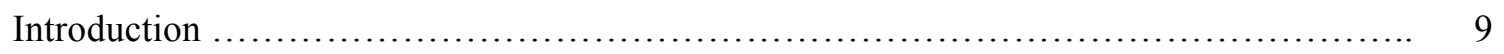

Behavioral Parent Training .................................................... 11

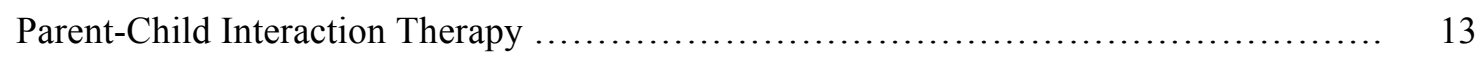

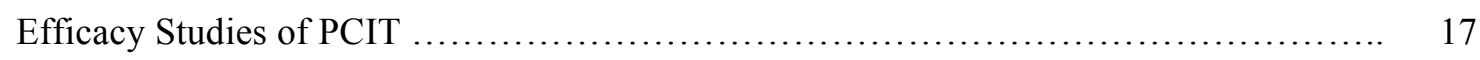

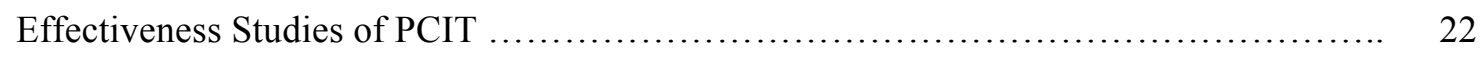

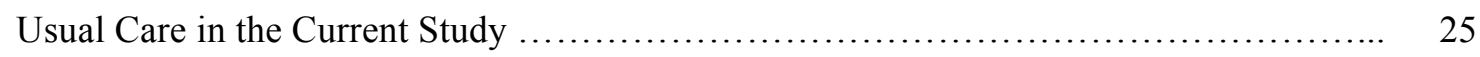

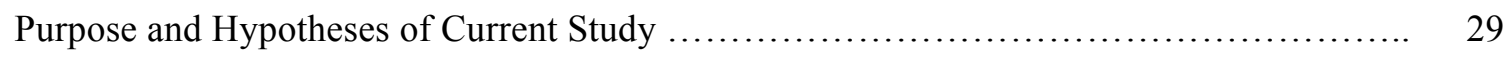

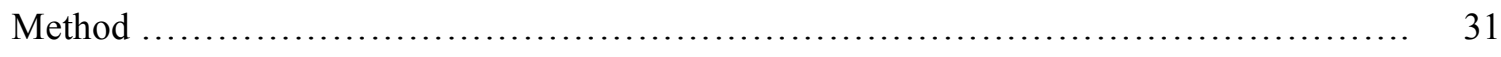

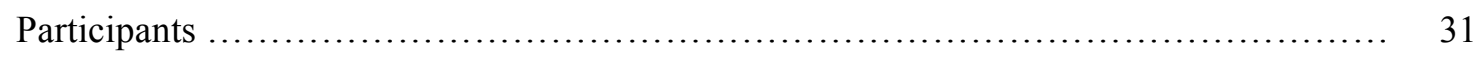

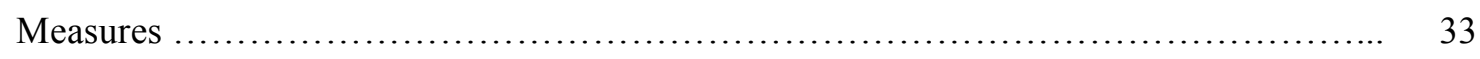

Procedures .......................................................... 37

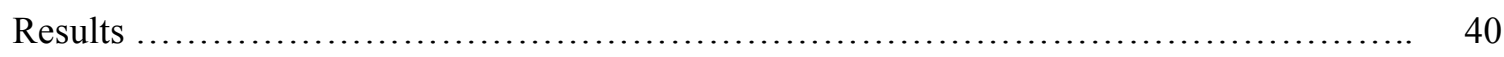

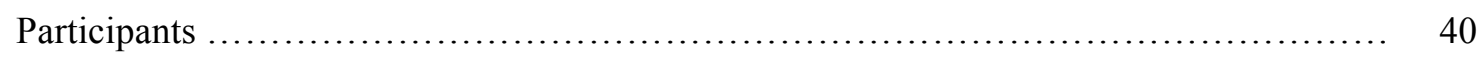

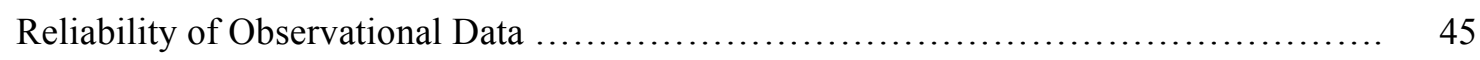

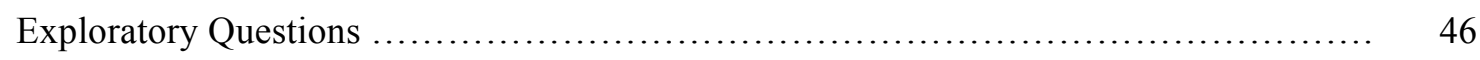

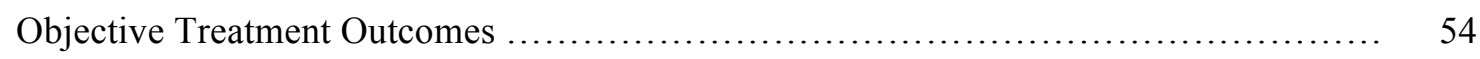

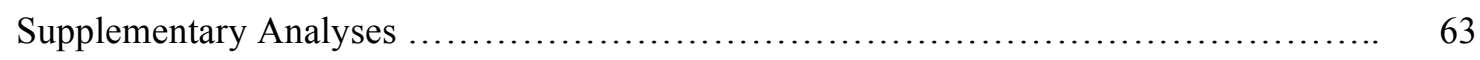

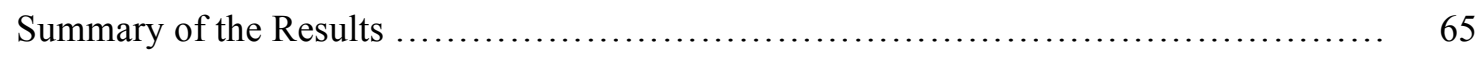

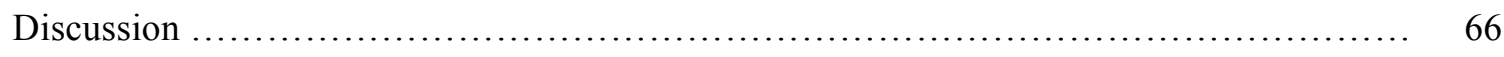

Differences in Dose between Groups ....................................... 66 


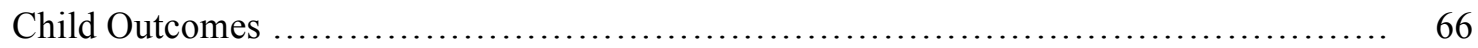

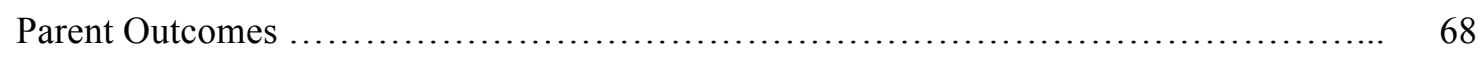

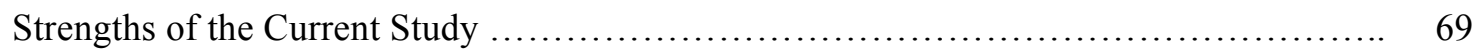

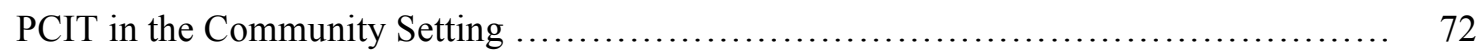

Usual Care: Behavioral Health Rehabilitative Services ........................... 78

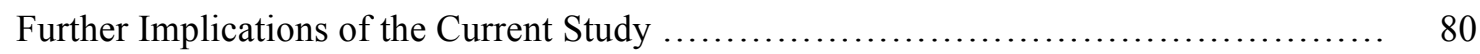

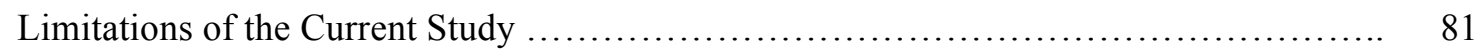

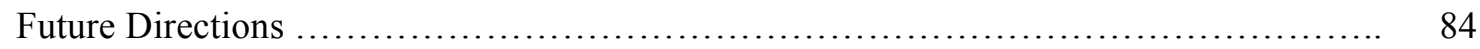

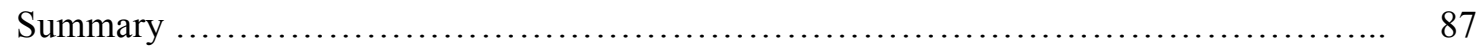

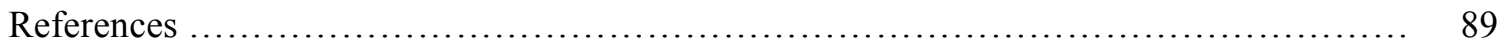

Tables

1 - Definitions, Examples, and Kappas for Categories of the DPICS ................. 100

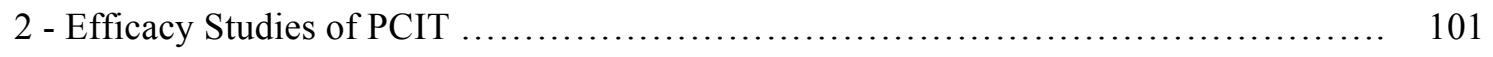

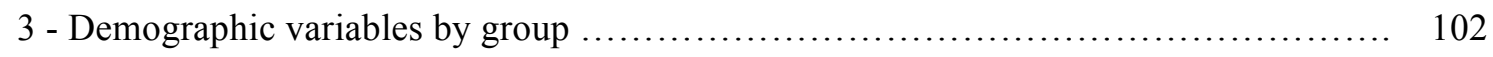

4 - Individual Participants’ Demographic Information, Diagnoses, Dose, and Outcomes

Ranked in Order of Greatest to Least Change on ECBI ....................... 103

5 - Description of Treatment and Fidelity .................................... 105

6 - Responses on the Qualitative Caregiver Satisfaction Survey .................... 106

7 - PCIT Therapists' Responses on Qualitative Survey ........................... 109

8 - PCIT Compared to UC: Test Statistics and $p$ Values of the

Interaction for Outcome Measures .................................... 112

9 - Cohen's d Effect Sizes from First to Second Assessment by Group and Comparison to

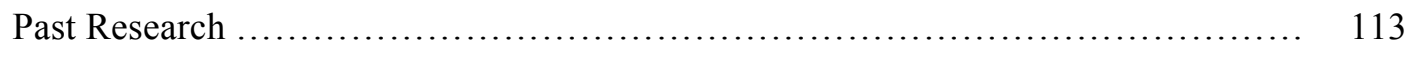

10 - "Pure" PCIT and "Pure" BHRS: Test Statistics and p Values of the

Interaction for Outcome Measures ...................................... 114 
11 - "Pure” PCIT and BHRS: Individual Participants' Demographic Information, Diagnoses, Dose, and Outcomes Ranked in Order of Greatest to Least Change on

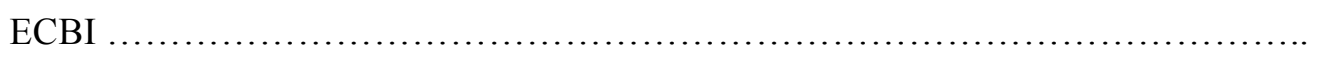

Figures

1 - Change on ECBI Raw Intensity Scale as Function of Billable Service Hours .......... 116

2 - Change on ECBI Raw Intensity Scale as Function of Billable Service Hours for PCIT 117

3 - Effect Sizes from the First to Second Assessment for the PCIT and UC Groups Across 118 Objective Outcome Measures

4 - Effect Sizes from the First to Second Assessment for the "pure" PCIT and "pure" UC Groups Across Objective Outcome Measures

5 - Child Improvement in Whole Sample 120

6 - Child Improvement in "Pure" Sample

Appendices

A - Caregiver and Child Information Form .

B - Therapist Information Form

C - Clinical Information Form

D - Therapist Report of Treatment Attendance and Completion

E - PCIT Therapist Follow-Up Survey

F - Caregiver Satisfaction Survey 136

G - PCIT Treatment Questionnaire 138

H - UC Treatment Questionnaire 
Effectiveness of Community- Delivered Parent-Child Interaction Therapy Compared to Treatment as Usual

A number of psychological interventions have empirical support for treating specific presenting complaints in child behavioral health. For example, numerous well-controlled studies with high internal validity indicate that Parent-Child Interaction Therapy (PCIT) is an efficacious treatment for child disruptive behavior disorders (e.g., McCabe \& Yeh, 2009; Nixon, Sweeney, Erickson, \& Touyz, 2003; Schuhmann, Foote, Eyberg, Boggs, \& Algina, 1998). Efficacy refers to research studies high in internal validity in which the investigator controls many aspects of treatment delivery including the sample selection, therapists, and setting (Hoagwood, Hibbs, Brent, \& Jensen, 1995; Weisz \& Jensen, 1999; Weisz, Jensen-Doss, \& Hawley, 2006). In contrast, effectiveness refers to studies in which efficacious treatments are delivered by clinicians in more natural settings (Hoagwood et al., 1995; Weisz \& Jensen, 1999; Weisz, Jensen-Doss, \& Hawley, 2006). An important goal in the development of evidence-based practice in child psychology is to demonstrate that interventions are both efficacious and effective (Weisz, JensenDoss, \& Hawley, 2005).

The majority of treatment outcome studies in child mental health fall into the category of efficacy research (Weisz et al., 2005). Meta-analyses of efficacy studies indicate that child therapies have moderately large effect sizes, but the conditions under which these treatments were tested are not typical of the conditions under which most therapy is provided (Weisz, Weiss, \& Donenberg, 1992; Weisz \& Jensen, 1999; Weisz, Jensen-Doss, \& Hawley, 2005; Weisz et al., 2006). For example, children recruited for studies may not be as severely impaired as children referred for treatment, and stricter inclusion and exclusion criteria in efficacy studies may result in samples with less comorbidity (Weisz et al., 1992; Weisz \& Jensen, 1999; Weisz et al., 2006). Therapists in efficacy studies are usually trained in the treatment immediately preceding the study, receive supervision during the study, and use a manual to promote treatment fidelity (Weisz et al., 1992; Weisz \& Jensen, 1999; Weisz et al., 2006). Efficacy studies often involve 
treatments with behavioral components whereas community-based treatments more often involve family and psychodynamic therapy (Weisz, Donenberg, Han, \& Weiss, 1995). Additionally, lab treatments usually rely on a narrow range of techniques to address a specific problem (Weisz \& Jensen, 1999). Because of important distinctions between efficacy and effectiveness research (e.g., greater severity/ dysfunction in clinically-referred children), concerns have been raised that university-based treatment outcome research leads to greater effect sizes than can be expected in community samples (Weisz et al., 1995).

More research is needed to examine the generality of treatments tested in efficacy studies (Garland, Hurlburt, Brookman-Frazee, Taylor, \& Accurso, 2010; Weisz et al., 1992; Weisz et al., 2005). Examination of usual care has revealed that practicing clinicians appear to use many evidence-based practices but to deliver them with low intensity (Garland, Brookman-Frazee, Hurlburt, Accurso, Zoffness, Haine-Schlagel, \& Ganger, 2010). Collaboration between researchers and community-based practitioners, along with the inclusion of qualitative measures of treatment acceptability, may be necessary to understand the effectiveness of evidence-based practices (Frazier, Formoso, Birman, \& Atkins, 2008; Hoagwood \& Kolko, 2009; Hohmann \& Shear, 2002). Effectiveness evaluations are necessary to determine the actual outcomes that can be expected when interventions are delivered by practicing clinicians working with clinic-referred children (Hoagwood et al., 1995; Weisz \& Jensen, 1999; Weisz et al., 2006).

To achieve high quality mental health services for most children, the first step is to determine that treatment yields positive outcomes in studies with high internal validity (Hoagwood et al., 1995; Weisz et al., 2005; Weisz et al., 2006). However, once research indicates that a treatment is efficacious, the therapy must be introduced to the community to assess its effectiveness (Hoagwood et al., 1995; Weisz et al., 2005). Consistent with the child psychotherapy treatment outcome literature at large, which is comprised almost entirely of efficacy studies (Weisz \& Jensen, 1999; Weisz et al., 2005), there have been few studies that have examined the outcomes of PCIT delivered by community clinicians (e.g., Pearl, Thieken, 
Olafson, Boat, Connelly, Barnes, \& Putnam, 2011; Phillips, Morgan, Hawthorne, \& Barnett, 2008), and these studies have significant methodological limitations. Thus, PCIT is an example of an efficacious treatment for which more research is needed to understand its effectiveness (Herschell, Calzada, Eyberg, \& McNeil, 2002b).

\section{Behavioral Parent Training}

Behavioral parent training (BPT) has emerged as the most appropriate evidence-based treatment for young children with disruptive behavior disorders (Kaminski, Valle, Filene, \& Boyle, 2008; McCart, Priester, Hobart, \& Azen, 2006). BPT has larger effect sizes than cognitive behavioral therapy for treating preschool-aged and school-aged youth with antisocial behavior (McCart et al., 2006). Common components of BPT programs are selective attention to appropriate child behavior, planned ignoring, use of commands, and time-out (Kaminski et al., 2008). BPT programs that include components to increase positive parent-child interactions, to effectively implement time-out, and in-session parental practice of skills with the child have larger effect sizes than BPT programs that do not include these components (Kaminski et al., 2008). Coaching was found to increase the effect sizes of parent training programs (Kaminski et al., 2008), which is similar to past research that has found that coaching increases teacher use of new strategies in the classroom (Showers \& Joyce, 1996). Modeling, homework, and roleplaying without the aforementioned components are not as effective (Kaminski et al., 2008).

Many BPT programs are based in the Hanf model (Reitman \& McMahon, 2013), which has two phases of treatment. The first phase focuses on building positive parent-child interactions and parental attention to appropriate behavior (Kaminski et al., 2008). The second phase focuses on increasing child compliance and decreasing inappropriate behavior through effective commands and time out (Kaminski et al., 2008). Common BPT programs based in the Hanf model include the Incredible Years (IY; Webster-Stratton, 1984; Webster-Stratton \& Hammond, 1990; 1997), Positive Parenting Program (Triple P; Sanders, Markie-Dadds, Tully, \& Bor, 2000), Defiant Children (Barkley, 1997), Helping the Noncompliant Child (HNC; McMahon 
\& Forehand, 2003), and PCIT. Defiant Children, HNC, and PCIT have been published in book form for clinicians. IY has been published in book form for parents (Webster-Stratton, 1992). It is unknown how many clinicians and parents have used strategies from these books and the benefits they have attained. However, Herschell and colleagues (2009) found that reading the treatment manual is not sufficient in training therapists in the skills of PCIT. The majority of published research in these programs is best characterized as efficacy research.

Triple P (Saunders et al., 2000) is a behavioral parent training program in which the effectiveness of dissemination has been examined. Triple $\mathrm{P}$ has been disseminated in the form of a television series and television documentary series (e.g., Sanders, Calam, Durand, Liversidge, \& Carmont, 2008; Sanders, Montgomery, \& Brechman-Toussaint, 2000). Parents who participated completed questionnaires before and after watching the media series. These parents reported reductions in child disruptive behavior and an increased sense of competence compared to a waitlist control group (Saunders et al., 2008); however, the impact of the television series on the population in general is unknown.

Large-scale dissemination of Triple P in Australia (e.g., Sanders, Ralph, Sofronoff, Gardiner, Thompson, Dwyer, \& Bidwell, 2008) and the US (e.g., Prinz, Sanders, Shapiro, Whitaker, \& Lutzker, 2009) has been examined. The parenting principles of Triple P were disseminated through local mass media (e.g., news segments, radio), "low-cost, high quality written resources" available in primary care physicians' offices and schools, and training of professionals in child welfare settings. Sanders and colleagues (2008) assessed effects through a phone interview with a random sample of households in the participating ten geographic areas. Compared to geographic areas that did not receive the dissemination of Triple $\mathrm{P}$, a significant decrease in clinically significant levels of behavioral and emotional problems was present (Sanders et al., 2008). Prinze and colleagues (2009) found significantly fewer substantiated cases of child maltreatment, out-of-home placements, and child maltreatment injuries in the counties that were randomized to receive dissemination of Triple $\mathrm{P}$. 
The effectiveness of Triple $\mathrm{P}$ indicates promising benefits of large-scale dissemination of BPT. Sanders and Kirby (2012) warn that population differences may produce differential benefits in large-scale dissemination efforts. They recommend continued involvement of key stakeholders, such as families and providers, in an iterative process to refine BPT to produce the most benefit.

\section{Parent-Child Interaction Therapy}

PCIT is a Behavioral Parent Training program. In the current study, PCIT is considered a specialized form of outpatient therapy. PCIT is based on the Hanf model of parent training in which parents are trained as therapists for their children (Eyberg, 1988; Eyberg, Nelson, \& Boggs, 2008). The first phase, called Child-Directed Interaction (CDI), focuses on building a strong positive relationship between the parent and child (McNeil \& Hembree-Kigin, 2011). The second phase, called Parent-Directed Interaction (PDI), focuses on increasing child compliance and decreasing aggression (McNeil \& Hembree-Kigin, 2011). Unique aspects of PCIT compared to other treatment programs are that parents are taught specific behaviors, their use of these behaviors is coded, and then they receive frequent in-vivo feedback on their skill acquisition through therapist “coaching” (Herschell, Calzada, Eyberg, \& McNeil, 2002a). Additionally, "mastery" criteria for each treatment phase provide goals and define criteria for treatment termination (Herschell et al., 2002a). During the first session of each phase, therapists outline and give the rationales for treatment during an initial didactic session that may last two hours (McNeil \& Hembree-Kigin, 2011). After this initial didactic session, therapists code the parent's skill acquisition and coach the parent to mastery criteria during approximately 12 50-60-minute sessions (McNeil \& Hembree-Kigin, 2011).

During CDI, the parent follows the child's lead and learns to use his or her attention selectively to increase prosocial behaviors and decrease inappropriate behaviors (Eyberg, 1988; McNeil \& Hembree-Kigin, 2011). Parents are taught four specific skills that constitute attention to appropriate and neutral behavior. These skills are labeled praise, reflections, behavioral 
descriptions, and imitation (Eyberg, 1988; Eyberg \& Funderburk, 2011). (See Appendix A for definitions and examples.) Lastly, parents are encouraged to enjoy the time with their child (Eyberg \& Funderburk, 2011). Together, these skills are referred to as the "PRIDE skills."

To maintain the child's lead, the parents refrain from placing demands on the child in the form of commands and questions (Eyberg, 1988; Eyberg \& Funderburk, 2011; McNeil \& Hembree-Kigin, 2011). Additionally, parents are to refrain from "negative talk," which is any negative evaluation of the child or child's behavior (Eyberg, 1988; Eyberg \& Funderburk, 2011; McNeil \& Hembree-Kigin, 2011). Collectively, commands, questions, and negative talk are called the "Avoid" skills. (See Appendix A for definitions and examples.) Instead of attending to misbehavior in the form of reprimands or directives, parents are coached to ignore the inappropriate behavior and redirect the child by enthusiastically describing their own play. Mastery criteria are providing ten labeled praises, ten reflections, ten behavioral descriptions, and fewer than three combined commands, questions, and/ or negative statements in a 5-minute observation (Eyberg \& Funderburk, 2011). When parents reach the mastery criteria, they progress to PDI (Eyberg \& Funderburk, 2011).

PDI focuses on increasing child compliance and decreasing aggression using a specific time-out procedure (Eyberg \& Funderburk, 2011; McNeil \& Hembree-Kigin, 2011). Parents learn to differentiate direct commands, those that tell the child what to do, from indirect commands, which ask or suggest that a child do something (Eyberg, 1988; Eyberg et al., 2005; see Appendix A for definitions and examples). Parents initiate the time-out sequence following noncompliance to a direct command (Eyberg, 1988; Eyberg \& Funderburk, 2011). Labeled praise for compliance is provided, such as "Thank you for listening." The parent follows noncompliance to a direct command with a warning statement (Eyberg \& Funderburk, 2011). At first, children only go to time-out for noncompliance to the warning statement. Time-out is in a chair and lasts for three minutes with a release contingency of five seconds of silence (Eyberg \& Funderburk, 2011). After this criterion is met, the child must comply with the original command 
and a second "follow-up" command as an overcorrection procedure (McNeil \& Hembree-Kigin, 2011). Following compliance to the follow-up command, the parent gives the child a labeled praise statement (Eyberg \& Funderburk, 2011).

Escape from time-out in the chair results in a one-minute time-out in a small room or area after which the child is placed on the time-out chair again to complete a full time-out in the chair (Eyberg \& Funderburk, 2011). In the clinic, time-out "rooms" are approximately 4-5 feet by 4-5 feet and may be separate rooms or areas in the therapy room with walls that are about 5 feet high. At home, time-out rooms are usually bedrooms or bathrooms from which all dangerous items have been removed (McNeil \& Hembree-Kigin, 2011). After the child has learned to stay on the time-out chair, house rules are introduced. For example, "No hurting" is a common house rule that addresses aggression. The parent chooses a few house rules, often a maximum of three, and breaking a house rule results in an immediate time-out (McNeil \& Hembree-Kigin, 2011).

The mastery criteria for PDI are at least $75 \%$ parental direct commands of total parental commands, $75 \%$ or greater child compliance to parental direct commands, and at least $75 \%$ "follow through" with labeled praise for compliance or the warning statement and time-out procedure for noncompliance when the parent gives at least four commands in five minutes (Eyberg \& Funderburk, 2011). Additionally, families remain in treatment until parent report of child behavior on the Eyberg Child Behavior Inventory (ECBI, Eyberg \& Pincus, 1999) indicates that child problem behavior is well below the clinically significant range ( $T$ score no greater than 55; Eyberg \& Funderburk, 2011).

Certain assessment measures for PCIT are indicated for use based on the PCIT manual (Eyberg \& Funderburk, 2011). The ECBI is a parent-report measure of common child behavior problems at home, and the manual (Eyberg \& Funderburk, 2011) indicates that parents complete this measure at each session. Therapists graph the Intensity Scale, indicating the frequency of disruptive behaviors at home, each session and periodically show the graph to the parents as a measure of treatment success. Additionally, therapists observe parent-child interactions at the 
beginning of all coaching sessions using the Dyadic Parent-Child Interaction Coding System (DPICS; Eyberg, Nelson, Duke, \& Boggs, 2005) to measure parent acquisition of skills and guide coaching. Thus, evidence-based assessment including both parent report of child behavior and therapist direct observation of parent and child behavior is included in the treatment manual.

PCIT International, an organization created to promote quality training in PCIT, has established guidelines for the training of community therapists in PCIT (PCIT International, 2013). Therapists must have earned a master's degree or higher and be able to provide therapeutic services in their state (e.g., licensed mental health professional, supervised by a licensed mental health professional; PCIT International, 2013). Typically, community therapists complete a 40-hour initial PCIT training in five days, which includes theoretical foundations, practice coding observations, practice coaching with families, and a review of the 2011 PCIT Protocol. After the initial training, therapists receive continued training with a focus on case experience and therapist skill review (e.g., live or videotaped observations of sessions; PCIT International, 2013). It is common for therapists to participate in monthly telephone case consultations. According to PCIT International (2013), continuing education can take the form of co-therapy, online training, workshops, or a combination of these methods. Some therapists participate in a follow-up 14-hr, two-day advanced PCIT training about six months following their initial training. This advanced training addresses case questions that have arisen as the therapists have begun implementing PCIT, and therapists review videotapes of their PCIT as part of the workshop.

PCIT International has also established requirements for those who are permitted to train others in PCIT. Trainers are divided into the three categories "Level I" Trainer, "Level II" Trainer, and "Master" Trainer. Level I Trainers have met the requirements to be a "PCIT therapist" and have completed at least four PCIT cases. They are permitted to train other therapists within their "program" (e.g., agency, clinic) who they directly supervise and with whom they may directly observe or conduct co-therapy. Level II Trainers are permitted to train 
therapists outside of their agency within their geographic region (e.g., state, metropolitan area).

Level II Trainers must have met certain requirements for graduate level training including coursework in behavioral principles, child development, assessment, and intervention.

Additionally, Level II Trainers must demonstrate that they have significant experience using PCIT with families, successfully trained therapists within their "program," and be involved in the PCIT community (e.g., participation at conferences; PCIT International, 2013). Master Trainers are allowed to train nationally and internationally. They have met all the requirements of PCIT Therapists, Level I Trainers, Level II Trainers, and are intended to be leaders in the dissemination of PCIT and integrity to the PCIT model. Master Trainers may supervise Level I and Level II Trainers. These guidelines for training are intended to improve training in and fidelity to the PCIT model.

\section{Efficacy Studies of PCIT}

Many well-controlled studies from independent researchers have found significant improvements in parent-reported and observed child behavior following PCIT (i.e., Eyberg et al., 2008; Nixon et al., 2003; Schumann et al., 1998). PCIT has been characterized as a "probably efficacious" treatment for young children with disruptive behavior disorders (Eyberg, Nelson, \& Boggs, 2008) based on criteria set by Chambless and colleagues (1998). PCIT has not met criteria for a "well-established" treatment because only one study has examined the outcomes of PCIT compared to a treatment control group (e.g., McCabe \& Yeh, 2009). Some studies indicate that the positive outcomes associated with PCIT maintain for at least six years following the completion of treatment (Eyberg, Funderburk, Hembree-Kigin, McNeil, Querido, \& Hood, 2001; Hood \& Eyberg, 2003), generalize to the school setting (McNeil, Eyberg, Eisenstadt, Newcomb, \& Funderburk, 1991), and generalize to the home (Naik-Polen \& Budd, 2008). Over the past 30 years, the procedures of PCIT have become more defined and PCIT has been more rigorously tested (Herschell et al., 2002b). 
Efficacy studies of PCIT have increased in methodological rigor over time. Early examinations found significant improvement in mother-child dyads using pre-post treatment designs (e.g., Eisenstadt, Eyberg, McNeil, Newcomb, \& Funderburk, 1993; Eyberg \& Robinson, 1982) and pre-post treatment designs with a waitlist control group (e.g., Schuhmann, Foote, Eyberg, Boggs, \& Algina, 1998; Nixon, Sweeney, Erickson, \& Touyz, 2003). McCabe and Yeh (2009) is the only study known to this author to compare PCIT to UC. McCabe and Yeh (2009) found that both PCIT and UC resulted in clinically significant improvements in child behavior; however, PCIT resulted in significantly larger improvements than usual care (UC). See Table 2 for a comparison of these studies.

The results of these studies indicate that parent-report measures reveal positive treatment outcomes. Parent report on the ECBI (Eyberg \& Pincus, 1999) and CBCL (Achenbach, 1992) reveal a reduction in frequency of parent reported behavior problems to the normative range at post treatment (Eisenstadt et al., 1993; Eyberg \& Robinson, 1982; McNeil, et a., 1991; McCabe \& Yeh, 2009; Nixon et al., 2003; Schuhmann et al., 1998). Observed child compliance with parent commands significantly improved from pretreatment and compared to a wait list control group (Eisenstadt et al., 1992; McNeil et al., 1992; Nixon et al., 2003). Observations of parentchild interactions revealed significant increases in praise, overall decreases in commands, and reductions in criticism compared to waitlist controls (Nixon et al., 2003; Schuhmann et al., 1998). McCabe and Yeh (2009) found significantly more composite "Do" skills (e.g., labeled praise, reflection, behavior description) and fewer composite "Avoid" skills (e.g., commands, questions, negative talk) for parents enrolled in PCIT in all observed situations compared to UC. PCIT significantly decreased parenting stress from pretreatment and compared to parents in waitlist control groups (Eisenstadt et al., 1993; Schuhmann et al., 1998; McCabe \& Yeh, 2009). The results of the studies indicate that parents' behavior during interactions with their children changes compared to pretreatment, waitlist controls, and UC. Additionally, parents report 
significantly larger reductions in child disruptive behavior and parenting stress compared to waitlist controls and UC.

Effect sizes for PCIT efficacy studies have been large (Thomas \& Zimmer- Gembeck, 2007). Nixon and colleagues (2003), who compared PCIT to a waitlist control, reported effect sizes with Cohen's $d$ ranging from .96 to 1.78 for observational measures and .57 to 1.13 for the parent report measures. McCabe and Yeh (2009), who compared PCIT to usual care delivered by community therapists, reported effect sizes with Cohen's $d$ to range from .7 to 1.55 for observational measures and 1.04 to 2.14 for the former parent-report measures. McCabe and Yeh (2009), who reported only the Total Stress score, found a large effect size in the PCIT group (Cohen's $d=1.04$ ), a moderate effect size in the UC group (Cohen's $d=.57$ ), and significantly lower parenting stress scores for the PCIT group compared to pretreatment. Thus, efficacy studies of PCIT have found large effects for observational measures and moderate to large effects for parent-reported measures.

Efficacy studies have begun testing the limits of PCIT by examining the outcomes of PCIT with different populations. Initial studies have demonstrated that PCIT is a promising intervention for improving compliance in children with comorbid mental retardation (Bagner \& Eyberg, 2007) and autism (Masse, 2010; Solomon, Ono, Timmer, \& Goodlin-Jones, 2008). Other researchers are exploring the outcome of PCIT in populations with complex family stressors, such as families in which domestic violence occurs (Borrego et al., 2008). Some have added components to the original procedure to treat depression (Lenze et al., 2011) and separation anxiety (Choate, Pincus, Eyberg, \& Barlow, 2005). Many studies indicate that PCIT is efficacious in improving parenting skills and child behavior with parents who have had previous reports of physical abuse (Borrego, Urquiza, Rasmussen, \& Zebell, 1999; Chaffin, Silvosky, Funderburk, Valle, Brestan, Balachova, Jackson, Lensgraf, J., \& Bonner, 2004; Chaffin, Valle, Funderburk, Gurwitch, Silovsky, Bard, McCoy, \& Kees, 2009; Timmer, Urquiza, Zebell, \& McGrath, 2005). PCIT shows promise in improving the behavior of children in foster care when 
foster parents are trained (McNeil, Herschell, Gurwitch, \& Clemens-Mowrer, 2005; Timmer, Urquiza, \& Zebell, 2006). These studies indicate that PCIT may be effective with children and families who present with comorbidity.

Adapted and modified forms of PCIT have shown promise as well. Briefer versions of PCIT have yielded significant improvements in child behavior (e.g., Berkovits, O’Brien, Carter, \& Eyberg, 2010; Nixon et al., 2003; Pade, Taube, Aalborg, \& Reiser, 2006). Positive outcomes have also occurred when in-home coaching is provided in addition to clinic-based therapy sessions (Timmer, Zebell, Culver, \& Urquiza, 2010), and PCIT shows promise in being effective when delivered entirely in the home (Ware, McNeil, Masse, \& Stevens, 2008). Lastly, the studies by Lyon and Budd (2010) and McCabe and Yeh (2009) indicate that PCIT has promising outcomes when implemented by doctoral student therapists in community mental health clinics. Results of studies with non-standard formats and delivery locations suggest that PCIT may be effective in settings more typical of usual clinical care.

The efficacy studies have maintained high fidelity to procedures described in the PCIT manual. Treatment integrity has been reported at 99.6\% (Nixon et al., 2003), 97\% (Schumann et al., 1998), and 82\% (McCabe \& Yeh, 2009). McCabe and Yeh (2009) reported the lowest treatment integrity at $82 \%$, and this study took place in a community clinic. In the PCIT manual, each session (e.g., CDI Teach, CDI First Coach, CDI Second Coach, etc.) has an accompanying treatment integrity checklist. Following the session, the therapist checks whether or not each item on the checklist occurred. The integrity for any given session is the number of checks for a given action (e.g., coach PDI 15-20 minutes) indicating that it occurred divided by the total possible number of actions. In these studies, all sessions had been videotaped, and a second person watched the videotape and completed the integrity checklist for a certain percentage of sessions as a reliability check.

These studies of PCIT strongly suggest that PCIT is efficacious in improving parent and child functioning. Wait list comparison groups indicate that treatment gains are not due to 
maturation (e.g., Schuhmann et al., 1998; Nixon et al., 2003). Assessment has been multimodal incorporating both standardized parent-report measures and direct observations of parent-child interactions to examine treatment effects. Reliability coding and sufficient inter-rater reliability have supported observational data. Lastly, treatment fidelity has been carefully examined, and therapists have received extensive supervision to maintain high fidelity to the treatment model. High treatment integrity is corroborated by reliability of treatment integrity when examining videotapes of the therapy sessions. These experimental strengths increase the internal validity of these studies.

However, the efforts to ensure high internal validity create a setting that is not typical of usual treatment. Many characteristics of these studies differ from usual treatment in community clinics. The therapists in these studies were graduate students or interns in clinical psychology or social work (see table in Appendix B). Graduate students delivering therapy as part of an efficacy trial may be more motivated to have high treatment integrity than typical clinicians (Weisz et al., 1995). For example, Nixon and colleagues (2003) describe reading relevant portions of the treatment manual before each session (p. 253), but most therapists in community settings do not prepare as extensively for each session (Hoagwood et al., 1995). Additionally, most of the efficacy studies took place in university-based training clinics (for exceptions, see McCabe \& Yeh, 2009, and Lyon \& Budd, 2010). Thus, the therapists and physical location are not typical.

Also, many of these studies recruited participants (e.g., Nixon et al., 2003), which may result in less severity in the presenting problems than in clients who seek treatment (Weisz et al., 1992; Weisz \& Jensen, 1999). While the inclusion criteria of these studies indicated that behavior was severe enough to warrant a diagnosis of Oppositional Defiant Disorder (American Psychiatric Association, 2000), or related disorder, these studies had exclusion criteria that may not reflect the comorbidity often present in usual care settings (Weisz et al., 1992). For example, Nixon and colleagues (2003) excluded children whose behavior problems were "because of" 
trauma, organic pathology, or physical or mental deficits (p. 252). Research indicates that severity of dysfunction is a key difference between research and community-based therapy (Weisz et al., 1995). These differences between clinicians, treatment settings, severity in presenting concerns, and complexity of clients' presenting problems may limit the generality of the results of these efficacy studies to the usual care settings.

\section{Effectiveness Studies of PCIT}

In recent years, a growing number of studies have examined the effectiveness of PCIT delivered by community therapists (Abrahamse et al., 2012; Galanter, Self-Brown, Valente, Dorsey, Whitaker, Bertuglia-Haley, \& Priesto, 2012; Lanier et al., 2011; Lanier, Kohl, Benz, Swinger, \& Drake, 2014; Pearl et al., 2011; Phillips et al., 2008). Outcomes suggest that PCIT was effective in decreasing parent-reported child disruptive behavior on the ECBI (Abrahamse et al., 2012; Galanter et al., 2012; Lanier et al., 2011; Pearl et al., 2011; Phillips et al., 2008). Additionally, parenting stress decreased (Lanier et al., 2011; Pearl et al., 2011; Phillips et al., 2008). Parent-child observations indicated increased parental use of the PRIDE skills (Galanter et al., 2012; Pearl et al., 2011) and increased child compliance (Galanter et al., 2012). Lanier and colleagues (2014) suggest the effectiveness of PCIT in reducing future reports of child maltreatment.

The effectiveness studies contribute to the PCIT literature at large by examining PCIT in a natural setting. For example, therapists were master's level clinicians (e.g., Lanier at al., 2011; Pearl et al., 2011) rather than doctoral students who served as therapists in efficacy studies of PCIT. Services were provided in community mental health centers (e.g., Abrahamse et al., 2012; Lanier et al., 2011; Pearl et al., 2011; Phillips et al, 2008) or the home setting (e.g., Galanter et al. 2012; Lanier et al., 2011), which were typical of the agencies providing services, rather than in university-based training clinics. Additionally, minimal exclusion criteria may have created samples that were more typical of usual care settings. For example, $89 \%$ of the children in the study by Pearl and colleagues (2011) had a history of trauma in addition to disruptive behavior, 
and Phillips and colleagues (2008) described their sample as having "multiple presenting problems" (p. 713). The effectiveness literature shows promise that PCIT can be an effective therapy for young children with disruptive behavior in the community setting. Additionally, the effectiveness literature suggests that therapists trained in a less intensive manner than the doctoral students in efficacy studies can have good treatment outcomes in PCIT.

Another valuable contribution of the effectiveness studies is that they provided important information regarding treatment completion in a community setting. Treatment completion has been reported at 31\% (Lanier et al., 2011), 37\% (Pearl et al., 2011), 55\% (Abrahamse et al., 2012), 65\% (Galanter et al., 2012), and 88\% (Phillips et al., 2008). Phillips and colleagues (2008) report the highest retention rate; however, $15.4 \%$ of families were not required to complete PDI because it was not deemed clinically necessary. Phillips and colleagues (2008) reported that $73 \%$ of families completed both CDI and PDI. The effectiveness literature provides some information regarding when dropout occurs in PCIT. The majority of families dropped out during CDI. Specifically, $54 \%$ to $58 \%$ of the total sample dropped out during CDI, and $0 \%$ to $15 \%$ of the total sample dropped out occurred during PDI (Lanier et al., 2011; Pearl et al., 2011; Phillips et al., 2008). Abrahamse and colleagues (2012) reported that $64 \%$ of the families dropped out within the first 10 sessions of PCIT. Lanier and colleagues (2011) found no difference in drop out rates between delivery in the clinic and home settings. The completion rates in the effectiveness studies are similar to the dropout rates of $57 \%$ to $74 \%$ reported in the efficacy literature (McCabe \& Yet, 2009; Nixon et al., 2003). It seems that PCIT is effective for families who complete PCIT; however, about $50 \%$ of families do not complete PCIT. It is unknown whether the drop out rate in PCIT is typical to dropout rates from therapy in general.

These effectiveness studies also provided information regarding the typical length of treatment for PCIT in a community setting. Those who completed PCIT reportedly attended an average of 12.2 session (Phillips et al., 2008), 17 sessions (Lanier at al., 2011), and 17.4 sessions (range 10 to 38; Abrahamse et al., 2012). Most of the efficacy studies reported that clients 
attended 13-14 weekly sessions (Eisenstadt et al., 1993; McNeil et al., 1991; Nixon et al., 2003; Schuhmann et al., 1998). Abrahamse and colleagues (2012) reported that families typically completed 10 sessions of CDI and 7 sessions of PDI. It appears that families who received PCIT at the community agencies took longer, on average, to complete treatment than did participants in efficacy studies. More sessions to complete treatment may be needed for a variety of reasons related to the sample (i.e., more severe presenting concerns, more complexity in presenting concerns, comorbidity) and therapists' training (i.e., integrity to the model, quality of coaching, accuracy of coding).

None of the effectiveness studies have examined treatment fidelity in the community setting. In contrast, the graduate student therapists in PCIT efficacy studies completed treatment fidelity checklists, and research assistants reviewed videotapes of therapy sessions to provide reliability data. These procedures may have been too time-consuming for community therapists. Travis and Brestan-Knight (2012) retrospectively reviewed the treatment fidelity of the first tape submitted to the PCIT trainer as part of the community therapists' ongoing training in the PCIT model. Fidelity was examined for six key sessions. The mean fidelity score for each session was as follows: $90.52 \%$ for CDI Teach, $80.17 \%$ for the first CDI Coach, $92.31 \%$ for PDI Teach, $82.15 \%$ for the first PDI Coach, $91 \%$ for House Rules, and $87.76 \%$ for Public Behavior. These results indicated that community therapists had relatively high treatment fidelity. Nonetheless, given that these data are from tapes submitted to the PCIT trainer for review, it is possible that therapists submitted tapes with perceived high fidelity. Also, these therapists had recently been trained in the model, and fidelity may drift over time and as they gain experience in the model. It is unknown whether differences in treatment fidelity could play a role in the longer treatment duration found in the community effectiveness evaluations.

The effectiveness studies of PCIT provide valuable descriptions of PCIT and its effectiveness in the community setting. However, effectiveness studies of PCIT have weaker experimental designs. For example, most of the effectiveness studies have relied on retrospective 
pre-test/ post-test designs without a comparison group. Efficacy research has indicated that PCIT is superior to no treatment (McNeil et al., 1991; Nixon et al., 2003; Schuhmann et al., 1998); however, it remains unknown whether PCIT in the community is superior to the effects of other treatments in the community setting. Abrahamse and colleagues (2012) compared the parent report of child behavior in the PCIT families to a group of non-clinical children recruited from the community, which indicated that the passage of time alone could not account for the treatment gains found in PCIT. The dependent measures in the effectiveness studies primarily have been standardized parent-report measures (e.g., Lanier et al., 2011; Abrahamse et al., 2012; Phillips et al., 2008) rather than corroborating treatment outcomes with observational measures. Pearl and colleagues (2011) and Galanter and colleagues (2012) corroborated the parents' report with observational data collected in-session by the families' therapists. A family's therapist is not an unbiased coder, and the accuracy of the observational coding remains questionable without reliability data. Thus, the literature examining the effectiveness of PCIT in the community setting would be strengthened by inclusion of a usual care comparison group, multimodal assessment of both parent and child behavior, observational coding by independent assessors, reliability data, and description of treatment fidelity.

\section{Usual Care in the Current Study}

In the state where the current study took place, mental health care is divided into levels of care that include outpatient therapy, community-based enhanced mental health services, day treatment, residential treatment, and inpatient psychiatric hospitalization. Behavioral Health and Rehabilitative Services (BHRS) and Family-Based Mental Health Services (FBMHS) are types of community-based enhanced mental health services. When a family initiates therapy services, they may schedule an intake evaluation at an outpatient clinic. Alternatively, they may receive an evaluation for the medical necessity of a community-based enhanced mental health service (e.g., BHRS, FBMHS) called a "Best Practice” evaluation (Pennsylvania Department of Public Welfare, 2001). Any parent, child, or family may present to an outpatient clinic and receive 
therapy services. However, a Best Practice Evaluation is required to access community-based enhanced mental health services (Pennsylvania Department of Public Welfare, 2001).

Psychiatrists, psychologists, and psychological assistants who are supervised by psychologists can conduct Best Practice Evaluations (Pennsylvania Department of Public Welfare, 2001). These evaluations include a clinical interview with the parent and child, informal behavioral observations of the child, and administration of a standardized parent-report measure. In general, if the evaluator determines the need for BHRS or FBMHS, a "prescription" for the service is written with sufficient justification for the service, and the insurance company reviews this request. Prescriptions are written for the length of a specific "Plan of Care," which may be as long as 4, 6, or 12 months. To continue receiving services after the completion of a prescribed plan of care, the treatment team (i.e., family, therapists, evaluator) convenes for an evaluation of the medical necessity of continued care at the level of BHRS or FBMHS (Pennsylvania Department of Public Welfare, 2001).

Initial and Continued Care Best Practice Evaluations consist of examination of the domains of strengths (i.e., motivation, interests, skills across domains, family supports), concerns (e.g., clinical basis for current evaluation), family (i.e., family composition, family mental health history), school (i.e., current placement, behavioral concerns in school), community (i.e., use of leisure time, religious involvement, employment history, nature of neighborhood), peer relationships (i.e., frequency and quality of contact with peers), drug and alcohol use (i.e., current and past use by child and family), medical and developmental history, trauma history, and legal concerns (i.e., custody, past arrests and incarceration; Pennsylvania Department of Public Welfare, 2001). Observational data collected by the child's therapeutic staff may be presented at evaluations for Continued Care. Lastly, the evaluator administers a standardized parent-report measure. For cases in which disruptive behavior is the presenting concern, this measure would most likely be the Child Behavior Checklist (Achenbach, \& Rescorla, 2000; Achenbach \& Rescorla, 2001) is commonly administered. Information gathered from the interview, informal 
behavioral observations, and standardized report measures are used to establish the presence of dangerous, destructive, or debilitating behavior that indicates the medical necessity of BHRS or FBMHS.

In BHRS, the primary focus of therapy is the child, and parents are considered in relation to the child (Pennsylvania Department of Public Welfare, 2001). The techniques of therapy depend on the past training and history of the therapist. Therapists may have titles such as "Mobile Therapist (MT)," or "Behavior Specialist Consultant (BSC).” Mobile Therapists and Behavior Specialist Consultants are master's level clinicians who are licensed mental health providers or are unlicensed and thus supervised by licensed mental health providers. A child may also have a Therapeutic Support Staff (TSS) who is typically a person who has attended some college or has earned a bachelor's degree. The Mobile Therapist provides individual and family therapy services in the home for 2 to 4 hours per week. Behavior Specialist Consultants consult across settings including the home, school, and community, based on client need. In particular, the Behavior Specialist Consultants develop behavior intervention plans to address behavioral concerns and then supervise the Therapeutic Support Staff as they implement the plans. The Therapeutic Support Staff can provide services across the home, school, and community settings, as needed (Pennsylvania Department of Public Welfare, 2001), and the "script" may include a "dose" of Therapeutic Support Staff from 3 to 20 hours per week. The Therapeutic Support Staff's responsibilities may include modeling therapeutic responses to child behavior, helping the child participate in specific community activities (e.g., organized sports), facilitating positive peer interactions, helping the child calm down when angry, and facilitating successful problem-solving and decision-making (Pennsylvania Department of Public Welfare, 2001). Identified "pitfalls" of BHRS include "over-focusing" on the child, lack of therapy with the whole family, and potential over-utilization of services (Pennsylvania Department of Public Welfare, 2001).

In FBMHS, the primary focus of therapy is the family unit including the child, siblings, and parents (Pennsylvania Department of Public Welfare, 2001). A co-therapy team provides 
therapy in the home or community based on family systems approaches, such as Structural Family Therapy (Pennsylvania Department of Public Welfare, 2001). Families can access their treatment team 24 hours per day on 7 days of the week (Pennsylvania Department of Public Welfare, 2001). A prescription for FBMHS is more likely to occur when more than one child in the family is in need, a parent has identified significant mental health concerns, BHRS has been unsuccessful, or when a child is transitioning back into the home from an out-of-home placement (e.g., foster care, residential treatment facility; Pennsylvania Department of Public Welfare, 2001). Identified "pitfalls" of FBMHS include "under-focusing" on the child's symptoms and lack of assistance with the transition after FBMHS is completed (Pennsylvania Department of Public Welfare, 2001).

To this author's knowledge, no published efficacy studies have examined the treatment outcomes of BHRS or FBMHS; however, Knapp and Reed (2010) provide a cursory review of dissertation abstracts and data presented in posters that have examined the effectiveness of BHRS in many agencies and regions of Pennsylvania. Knapp and Reed (2010) assert that these studies "suggest a general trend of effectiveness" (pg. 5). They acknowledge the methodological limitations of these studies, including lack of a comparison group, but they do not provide a full discussion of them. It is unclear if this general trend is statistically or clinically significant, and it is possible that improvement is due to maturation rather than treatment effects. Wraparound services in general have been associated with fewer out-of-home placements (e.g., psychiatric hospitalizations, residential care, foster care; Bruns, Burchard, \& Yoe, 1995; VanDerBerg, 1993). Over a 6-month period, Toffalo (2000) found a medium effect size (Cohen's $d=.50$ ) in improvements in child behavior as measured by the Total Score on the CBCL (Achenbach \& Rescorla, 2000; 2001). Additionally, discrepancies between "prescribed" hours, meaning the number of hours the evaluator recommended, and actual hours received were not associated with treatment outcomes as measured by the CBCL Total Score (Toffalo, 2000). This means that 
families who had greater adherence to the recommended dose of treatment did not have greater improvements in child behavior than those with lower adherence to treatment recommendations.

BHRS and FBMHS may be best characterized as modalities of treatment in which a client may receive any variety of therapeutic techniques depending on the individual needs of the child and family. It is possible that clinicians working within these models may implement different aspects of evidence-based practices depending on their training.

\section{Purpose and Hypotheses of Current Study}

The purpose of the study was to examine the effectiveness of PCIT compared to UC when both treatments are delivered by practicing clinicians in a community setting. Exploratory questions about the delivery of PCIT in the community, characteristics of UC, and treatment satisfaction and acceptability were examined. Treatment outcomes using objective measures for a time period of approximately $7 \frac{1}{2}$ months were compared.

\section{Exploratory questions.}

What were the characteristics of UC and PCIT delivered by practicing clinicians?

1. What therapeutic practices were used in UC and how were they similar to and different from PCIT?

2. What was the fidelity to the PCIT model?

a. What components of PCIT had the best therapist adherence?

b. What components of PCIT had the lowest therapist adherence?

3. How many sessions of PCIT were completed?

4. How acceptable was PCIT in a usual care setting?

a. Were PCIT caregivers more satisfied with treatment than UC caregivers?

b. How acceptable was PCIT to therapists who provided this treatment?

\section{Aims and hypotheses.}

What are the treatment outcomes of PCIT compared to UC at the second assessment?

1. Did PCIT caregivers report greater improvements in child behavior than UC caregivers? 
a. It was hypothesized that caregivers would report significantly greater improvements in child behavior in the PCIT group than in the UC group. Past research has indicated that caregivers who receive PCIT in the community report improvements in child behavior (Galanter et al., 2012; Pearl et al., 2011; Phillips et al., 2008) and have reported larger improvements than usual care (McCabe \& Yeh, 2009).

2. Did PCIT caregivers demonstrate greater use of specialized parenting skills (e.g., praise, direct commands) than UC caregivers?

a. It was hypothesized that caregivers in the PCIT group, when compared to the UC group, would display a greater increase in "Do" skills (e.g., labeled praise, reflections, behavior descriptions), and decrease in "Avoid" skills (e.g., commands, questions, negative talk) during the observation of Child-Led Play. Past research has indicated that parents who have received PCIT demonstrate increases is "Do" skills and decreases in "Avoid" skills (Galanter et al., 2012; Pearl et al., 2011) and compared to usual care (McCabe \& Yeh, 2009).

b. It was hypothesized that caregivers in the PCIT group, when compared to the UC group, would display an increase in proportion of direct commands and increase in praise following child compliance during the observation of Parent-Led Play and Clean Up. Past research has indicated greater parental use of direct commands following PCIT (Galanter et al., 2012) and compared to usual care (McCabe \& Yeh, 2009).

3. Did children in the PCIT group have greater increases in observed compliance than children in the UC group?

a. It was hypothesized that children in the PCIT group, when compared to the UC group, would display a greater increase in observed percent compliance (number of commands followed by compliance divided by total commands). Past 
research indicated that children who receive PCIT in the community have improvements in child compliance (Galanter et al., 1012) and these improvements are greater than for those children who received usual care (McCabe \& Yeh, 2009).

4. Did caregivers in the PCIT group report greater decreases in total parenting stress than caregivers in the UC group?

a. It was hypothesized that caregivers in the PCIT group, compared to the UC group, would report a greater decrease in total parenting stress. Past research has indicated that parents who receive PCIT report greater decreases in parent stress compared to usual care (McCabe \& Yeh, 2009).

\section{Method}

\section{Participants}

Recruitment of therapists. To recruit "PCIT therapists," the researcher attempted to contact 22 therapists who had participated in two separate PCIT trainings in the 2 years preceding the initiation of this study. The researcher sent messages to the email addresses that the therapists had provided at their trainings. The researcher was able to correspond successfully with 13 of these therapists. Of the others who were unable to be contacted, it was known by colleagues that one had moved out-of-state and eight therapists did not respond to emails about the study. Of those therapists who were successfully contacted, two reported that they did not provide PCIT at that time, and three were engaged primarily in administrative activities. Three reported that they would use PCIT; however, they reportedly did not initiate PCIT with any families during the recruitment period of the study. Seven PCIT therapists referred participants to the study, and clients from six therapists were successfully enrolled in the study. Thus, a total of six PCIT therapists from four sites within two companies had patients who participated in the study.

To recruit therapists for the comparison group, the researcher met with clinical directors and therapists at 14 sites. All sites had therapists who provided therapeutic services in the form 
of outpatient therapy and Behavioral Health Rehabilitative Services (BHRS; "Wraparound"). One site provided Family-Based Mental Health Services in addition to outpatient therapy and BHRS. Therapists in the UC group had positions titled "Behavior Specialist Consultant," "Mobile Therapist," and/ or "Family-based Therapist." All had master's degrees and held the appropriate license or certification to provide clinical services in the state. Eight therapists referred participants who enrolled in the study. Additionally, two therapists with participating clients left their positions, and the three therapists who replaced them provided information about the participating clients. Thus, a total of eleven UC therapists from four sites across three companies participated in the study.

Recruitment of families. Families were eligible to participate in the study if (a) reduction of child disruptive behavior (e.g., aggression, defiance, tantrums) was a treatment goal and (b) the child was 2 to 7 years-old when he or she enrolled in the study. Thirty-five families signed forms to allow the researcher to contact them regarding the study. Thirty families enrolled in the study, with 15 families for each condition. One family for the comparison group did not respond after multiple phone calls and voicemails, and this family was not enrolled in the study. Four families referred for the PCIT group were unable to schedule a research assessment appointment before they had the third coaching session of PCIT (per research protocol), and these families were not enrolled in the study.

Assignment to condition. Families were assigned to a condition based on whether their therapist reported that he or she was trained in PCIT and reportedly using PCIT for the family. A family received PCIT or UC depending on knowledge of and availability of PCIT at the community agency to which the family presented for therapy. Additionally, familiarity with PCIT and availability of PCIT therapists influenced the likelihood that a family would receive PCIT. In agencies where Best Practice Evaluators were familiar with PCIT and knew of therapists trained in PCIT, families were more likely to receive PCIT. In agencies with less knowledge of PCIT, families were more likely to receive a recommendation for BHRS. Agency 
advertising (e.g., newspaper advertisements), outreach (e.g., school, primary care physicians, community fairs), and personal referrals (e.g., knew another family who had received satisfactory services at an agency) may have influenced where a family presented to receive services.

\section{Measures}

Caregiver and child information form. The caregivers completed a questionnaire regarding their age, sex, race, education level, annual income, occupation, marital status, employment status, number of people living in their home, child's sex, child's date of birth, child medication, and their relationship to the child (see Appendix A). They reported if they were currently or had ever been involved with Child Protective Services, and if they had received any counseling or therapeutic services in the past. Specific information regarding the type (e.g., outpatient, BHRS) of past therapy services was gathered.

Therapist information form. The therapists were asked to complete a brief questionnaire regarding the type of degree they held, and their years of clinical experience, theoretical orientation, gender, and race (see Appendix B).

Child diagnoses. Therapists were asked to report the billing and other diagnoses within one month of initiation of services and whether this child had ever been involved in Child Protective Services (see Appendix C).

Child Behavior Checklist. The Child Behavior Checklist (CBCL; Achenbach \& Rescorla, 2000, 2001) is a caregiver-report measure that assesses a variety of child symptoms of psychopathology. The CBCL consists of 99 and 112 problem items for the preschool-aged and school-aged child versions, respectively. The preschool-aged (children aged 1.5-5 years old) or school-aged (children aged 6-18 years old) versions were used depending on the age of the child. According to the manual, it is appropriate to compare scores across the preschool-aged and school-aged forms using $T$ scores (Achenbach \& Rescorla, 2000). The $T$ scores from the Internalizing and Externalizing scales were analyzed to examine externalizing and internalizing symptoms. 
One-week test-retest reliability has been found to be $r=.87$ to .92 for both scales across both versions (Achenbach \& Rescorla, 2000). Internal consistency with Cronbach's alpha has ranged from .87 to .90 for both scales across both versions indicating high internal consistency. The CBCL has been shown to distinguish between referred and non-referred children. Additionally, significant correlations have been found between the Internalizing Scale and depressive symptoms as well as between the Externalizing Scale and conduct problems (Achenbach \& Rescorla, 2000).

Eyberg Child Behavior Inventory. The Eyberg Child Behavior Inventory (ECBI; Eyberg \& Pincus, 1999) consists of 36 items and is a caregiver-report measure of the frequency of common behavior problems for children from 2 to 16 years old. The ECBI yields an Intensity score based on a 7-point Likert scale of the frequency of the behavior and a Problem score based on the caregiver's dichotomous report of whether or not the behavior is a problem. The Intensity raw score was analyzed in the current study to measure child disruptive behavior during the week prior to the assessment.

Test-retest reliability results has been reported to be $r=.86, .80$, and .75 for intervals of 3 weeks, 12 weeks, and 10 months, respectively. Cronbach's alpha for the most recent standardization sample was .95 indicating high internal consistency. Intensity Scores have been shown to have positive correlations with observed child negative affect, nonacceptance, and dominance (Webster-Stratton \& Eyberg, 1982). Additionally, Intensity Scores have been shown to have negative correlations with observed child positive affect (Webster-Stratton \& Eyberg, 1982). Treatment sensitivity has been demonstrated (e.g., Eisenstadt, et al., 1993; McNeil, et al.1991; Schumann, et al., 1998).

Parenting Stress Index: Short Form. The Parenting Stress Index: Short Form (PSI: SF; Abidin, 1990) is a 36-item caregiver-report measure that assesses stress related to parenting a child, and it is derived directly from the full-length Parenting Stress Index. The PSI:SF yields a Total Stress score. The Total Stress raw score was analyzed in this study. 
Six-month test-retest reliability has been measured at $r=.84$, and internal consistency has been high with a Cronbach's alpha of .91. The correlation between the short form and long form is $r=.94$. According to Abidin (1995), the PSI:SF relies on the validity of the full length PSI because it is derived from and has high positive correlations with the full length PSI. Treatment sensitivity of the full length PSI was demonstrated in a sample of aggressive parents (Acton \& During, 1992), and the PSI:SF was sensitive to treatment in the studies by Pearl and colleagues (2011) and by Phillips and colleagues (2008).

Dyadic Parent-Child Interaction Coding System- ${ }^{\text {rd }}$ Ed. The Dyadic Parent-Child Interaction Coding System, Third Edition (DPICS- III; Eyberg, et al., 2005) is an observational coding system that assesses caregiver and child behavior in the three structured situations of Child-led Play, Parent-led Play, and Clean Up. Reliability and validity have been shown in videotaped observations. One-week test- retest reliability has been measured at $r=.34$ to .57 for parent praise and negative talk in Parent-Led Play and Clean Up (Eyberg, et al., 2005, p. 222). Inter-rater reliability with Cohen's kappa ranges from .46 to .90 across all categories (see Table 1 for definitions and reliability statistics). Treatment sensitivity has been demonstrated (e.g., Eyberg \& Robinson, 1982; Schuhmann, et al., 1998; Webster-Stratton \& Hammond, 1990).

For Child-led Play, a composite of parent "Do" skills and "Avoid" skills was analyzed, which is consistent with past literature (e.g., McCabe \& Yeh, 2009; Pearl et al., 2011). "Do" skills are labeled praises, reflections, and behavioral descriptions. "Avoid" skills are commands, questions, and negative talk. Parent-led Play and Clean Up were combined to provide codes for percent direct commands (direct commands divided by total commands), percent child compliance (compliance divided by total commands), and percent contingent praise (percent of compliance followed by a labeled praise within 5 seconds).

Therapist report of treatment attendance and completion. Therapists were asked to report information about treatment completion and attrition during the time period that elapsed between a family's assessment visits for the study. If the researcher was unable to contact a 
family for the second assessment, then therapists were asked to provide information in reference to the 7.5 months following the first assessment, as this corresponded with the average elapsed time between assessment points. Specifically, therapists were asked whether a family has met the treatment goal regarding child disruptive behavior ("completed" treatment), is in progress of meeting this goal, or has "dropped out" of treatment (the therapist has had no in-person or telephone contact with this family for the past 4 weeks) during the time period between the two assessment points. PCIT therapists were asked to report the total number of sessions attended, cancelled, and for which the family did not show up during the last 6 months (see Appendix D).

Therapy Attitude Inventory. The Therapy Attitude Inventory (TAI; Brestan, Jacobs, Rayfield, \& Eyberg, 1999) consists of ten items measuring treatment satisfaction. Caregivers rate items on a 5-point scale where 1 indicates dissatisfaction or worsening of symptoms and 5 indicates satisfaction or improvements in symptoms. For example, items include: "The major behavior problems that my child presented at home before the program started are at this time (a) considerably worse, (b) somewhat worse, (c) the same, (d) somewhat improved, or (e) greatly improved" and "Regarding techniques of disciplining, I feel I have learned (a) nothing, (b) very little, (c) a few new techniques, (d) several useful techniques, and (d) very many useful techniques." Cronbach's alpha for the TAI is .91 , and 4-month test-retest reliability is $r=.85$ (Brestan, Jacobs, Rayfield, \& Eyberg, 1999). The TAI has been found to result in significantly different total satisfaction scores between treatments (e.g., Eyberg \& Matarazzo, 1980).

Qualitative therapist report. PCIT therapists were asked to complete a follow-up survey (see Appendix E) approximately 7 months after beginning the study. Therapists were asked to respond to open-ended questions regarding PCIT.

Qualitative family report. At the second assessment, caregivers answered open-ended questions regarding their satisfaction with treatment (see Appendix F).

Treatment fidelity. PCIT therapists were asked to complete a PCIT Treatment Questionnaire for each session. A Treatment Questionnaire was created for this study that 
includes treatment components of PCIT (see Appendix G). PCIT treatment components were drawn from PCIT integrity checklists (Eyberg \& Funderburk, 2011) with consideration of central components based on Herschell and colleagues (2002a). For example, items included administering and reviewing the ECBI, giving and reviewing homework, coding parent-child interactions, and coaching parent-child interactions.

The researcher asked Behavior Specialist Consultants to complete the Treatment Description Questionnaire for UC. Items on this questionnaire were based on components of PCIT and were stated in non-PCIT specific language (see Appendix H). For example, items asked if a standardized parent-report questionnaire was administered, parent behavior was coded, child behavior was coded, and homework was provided and collected. The questionnaires were typically administered in an interview format to allow the therapist to ask clarifying questions about the meaning of questionnaire items, if possible, which was achieved by the researcher attending the monthly Behavior Specialist Consultant meeting for the agency. Behavior Specialist Consultants were instructed to think of a "typical" week when answering questions to describe treatment. An open-ended section was provided at the bottom to allow the therapist to describe the treatment without constraints.

\section{Procedures}

Assessment for families. All of the assessments occurred in the family's homes. The researcher obtained the caregivers' informed consent. If a child was 7 years old, the researcher obtained the child's assent to participate in the study. Caregivers then completed the Demographic Form, Child-Behavior Checklist (CBCL; Achenbach \& Rescorla, 2000; 2001), Eyberg Child Behavior Inventory (ECBI; Eyberg \& Pincus, 1999), and Parenting Stress Index (PSI; Abidin, 1995). The caregiver and child then participated in a 20-minute videotaped observation. This observation consisted of a 5-minute "warm-up" period and each of the three DPICS assessment situations of Child-led Play, Parent-led Play, and Clean Up (Eyberg, et al., 2005; Eyberg, \& Funderburk, 2011). The researcher read instructions from the PCIT manual 
(Eyberg \& Funderburk, 2011,p. 13) to guide the caregiver and child about the observation.

Following completion of the assessment, the caregiver received a \$20 gift card.

After 6.5 months had elapsed since the first assessment, the researcher began calling the caregiver to schedule the follow-up assessment. All of the follow-up assessments took place in the family's homes. Caregivers completed the CBCL, ECBI, PSI, Therapy Attitude Inventory (Brestan, et al., 1999) and the qualitative therapy satisfaction measure (see Appendix G). The caregiver and child then participated in a 20-minute videotaped observation. This observation consisted of a 5-minute "warm-up" period and each of the three DPICS assessment situations of Child-led Play, Parent-led Play, and Clean Up (Eyberg, et al., 2005; Eyberg, \& Funderburk, 2011). The researcher read instructions from the PCIT manual (Eyberg \& Funderburk, 2011, p. 13) to guide the caregiver and child about the observation. The caregiver received a $\$ 40$ gift card after completing the assessment.

Therapists were asked to provide information regarding the therapy for each family who participated in the study. These included the Clinical Information Form (see Appendix C), the Treatment Questionnaire (see Appendix G and Appendix H), and a Follow-Up Survey (see Appendix D). Information gathered included child diagnoses, impressions of the parent's mental health, involvement in CPS, treatment completion, progress toward treatment goals, and attendance. For a description of the treatment, refer to the Results section. Dropout was defined as (a) the client terminates services before meeting treatment goals or (b) the therapist has no inperson or telephone contact with the caregiver or child for the previous 4 weeks.

Assessment for therapists. If therapists were willing, they consented to provide demographic and professional information about themselves using the Therapist Information Form. Therapists who had received training in PCIT were asked to complete a PCIT survey (see Appendix E) regardless of whether they referred patients to the study.

Coding of observational data. The researcher trained undergraduate research assistants to count the frequency of specific parent and child behaviors using the definitions provided in the 
DPICS- III. Prior to coding of study observations, coders were trained in the coding system using The Abridged Workbook: Coder Training Manual for the Dyadic Parent-Child Interaction Coding System- $3^{\text {rd }}$ ed. (Fernandez, Chase, Ingalls, \& Eyberg, 2006). They were required to pass the included review quizzes with $80 \%$ or greater correct, and they were required to code three consecutive training videotapes with a kappa of .60 or greater with the researcher for each of the three situations prior to coding the study data.

Undergraduate research assistants and the researcher transcribed the videotaped observations. Undergraduate research assistants who were naïve to the treatment condition coded the observations using the DPICS- III (Eyberg, et al., 2005). The researcher served as the reliability coder. If kappas were below .60 for the dependent measures for a participant, discrepant codes were reviewed and the tape was re-coded. Seven observations of Parent-Led Play and eight observations of Clean Up were coded more than once to achieve sufficient reliability.

Treatment integrity. Treatment questionnaires provided by PCIT therapists and Behavior Specialist Consultants were summarized. Eight of nine PCIT therapists provided reports of sessions for 11 cases. A PCIT therapist did not complete the session questionnaires for two participants because they dropped out soon after enrolling in the study. Session summaries reflected two CDI Teach sessions, 31 CDI Coach sessions, seven PDI Teach sessions, and 22 PDI Coach sessions. Few teach sessions were submitted to the researcher. The sessions reflect approximately $47 \%$ of the PCIT sessions that occurred during the study. Six of 11 UC therapists completed the treatment questionnaire for 11 participants.

\section{Power Analyses}

Power analyses were conducted with G*Power (Faul, Erdefelder, Lang, \& Buchner, 2007). Based on McCabe and Yeh (2009), chosen because it had a UC comparison group and $82 \%$ treatment integrity, a medium effect size was predicted for the current study. With a medium effect size, an alpha level of .05 , and power of $.80, \mathrm{G}^{*}$ Power indicated that a total 
sample size of 30 was needed to find a significant interaction with a 2 (PCIT v. UC) X 2 (Assessment 1 v. 2) Repeated Measures Analyses of Variance (RM ANOVA). Thirty families completed the first assessment, and 25 families completed the second assessment. Thus, the current study did not achieve a power of .80 based on a medium effect size and alpha level of .05 .

\section{Missing Data}

Measures were reviewed for missing responses and caregivers were given the opportunity to complete the missing items. If an item was missing on a standardized measure, the mean of the scale for that participant was imputed.

\section{Results}

\section{Participants}

Demographic information for therapists. Fifteen therapists of the total 20 therapists across the two groups consented to provide their demographic information. All therapists were Caucasian females. Therapists had a median of 6.5 years of clinical experience $(M=10.48, S D=$ 9.77, range 6 months to 32 years) and had earned their master's degree. Therapists described their approach to therapy, and they were not limited to one response. Results were as follows: $28.6 \%$ cognitive behavioral, $23.8 \%$ applied behavior analysis, $9.5 \%$ trauma focused, $9.5 \%$ eclectic, $4.8 \%$ strength-based, $4.8 \%$ existential phenomenological, $4.8 \%$ family systems, and $4.8 \%$ dialectical behavioral.

Of the six PCIT therapists who referred participants to the study, four therapists had received a 40-hour workshop training within the past 12 to 24 months, had the opportunity to participate in monthly phone consultation, and received a 16-hour workshop training in the 6 to 18 months prior to the initiation of the study. These therapists had completed two to four PCIT cases before they enrolled the first participant in the study. Additionally, one of the PCIT therapists had trained three therapists in her agency in PCIT using the "in house" training model. Two of these therapists referred two patients to the study. These two therapists were undergoing in-house training during the course of the study and had not yet completed a PCIT case. Thus, 
PCIT therapists who participated in the current study had completed 0 to 4 PCIT cases before referring families to the study.

Demographic information for families. Refer to Table 3 for demographic information listed by group. Thirty caregiver-child dyads participated in the study. Fifty-seven percent of the children were male. A Pearson chi-square analysis revealed a significant difference in child sex between the groups, $X^{2}=6.65, p=.01$. In the PCIT group, ten children were female and five were male. In the UC group, twelve children were male and three were female. The average child age at the first assessment was 5 years 0 months (range two years nine months to seven years three months; $S D=1.15$ ), and an independent samples $t$ - test indicated no significant difference in child age between groups, $t=-.05, p=.96$. Caregivers identified $79 \%$ of the children as Caucasian, $7 \%$ as African-American, $7 \%$ as biracial African-American and Caucasian, and 7\% as biracial American Indian and Caucasian. Caregivers identified the child's ethnicity as "Latino/ Hispanic" for 7\% of the children.

The therapists provided diagnoses based on the Diagnostic and Statistical Manual of Mental Disorder IV Text Revision (DSM-IV-TR; American Psychological Association, 2000). As of the first assessment, $53 \%$ of the children had a diagnosis of a disruptive behavior disorder only (e.g., Oppositional Defiant Disorder, Attention Deficit/ Hyperactivity Disorder, Disruptive Behavior Disorder, Not Otherwise Specified), 23\% had a diagnosis of an Autism Spectrum Disorder (ASD; e.g., Pervasive Developmental Disability, Not Otherwise Specified, Asperger's Disorder, Autism), 7\% had a comorbid diagnosis of a disruptive behavior disorder and an ASD, $3 \%$ had a comorbid diagnosis of a disruptive behavior disorder and an anxiety disorder, and 13\% had another diagnosis. These other primary diagnoses included Bipolar Disorder (3\%), Personality Disorder, Not Otherwise Specified (3\%), Disorder of Childhood, Adolescence, or Infancy, Not Otherwise Specified (3\%), and Adjustment Disorder (3\%). An additional three children (10\%) were diagnosed with an ASD during the study. 
A chi-square analysis indicated no significant differences between groups with regard to whether the child had a diagnosis of a disruptive behavior disorder, $X^{2}=.0, p=1.0$, as the same number of children had been diagnosed with a disruptive behavior disorder in each group.

Additionally, a chi-square analysis indicated no significant different between group with regard to the number of children diagnosed with an ASD, $X^{2}=.159, p=.50$. Six children in the PCIT group and five children in the UC group had an ASD. Three of the six children in the PCIT group with ASD were diagnosed during the study, and two of these three transferred to BHRS during the study.

To examine potential differences in aggression between the groups, the three items on the ECBI that address aggression (e.g., hitting parents, physically fighting with siblings, physically fighting with peers) were summed and compared between the groups. As each item is rated on a Likert scale from 1 indicating never occurs to 7 indicating always occurs, the summed totals had a possible range of 3 to 21 . Independent samples $t$ tests indicated no significant differences between the PCIT group $(M=9, S D=4.22)$ and UC group $(M=10.93, S D=4.95)$ at the first assessment with regard to parent reported child aggression towards parents, peers, and siblings, $t$ $(28)=-1.15, p=.26$, as measured by parent report on the ECBI items for hitting parents, physically fighting with friends, and physically fighting with siblings.

Six children (20\%) were prescribed psychotropic medication, per parent report. Four children were prescribed one medication, and two children were prescribed two medications. Prescribed medications included Ritalin, Adderall, Tenex (guanfacine), Concerta, Kapvay, Depakote, and Risperdal (risperidone). One child (3\%) had a change in their ADHD medication during the course of the study, per parental report. At the first assessment, one child in the PCIT group and five children in the UC group were prescribed medication. A chi-square analysis indicated no significant difference between the groups on whether or not a child was prescribed medication, $X^{2}(1)=3.33, p=.07$. However, it should be noted that this analysis approached significance, and statistical power may not have been sufficient. One child (3\%) in the PCIT 
group began taking ADHD medication during the study, and no children in the UC group initiated or discontinued use of medication during the study, both per parental report.

Ninety-seven percent of participating caregivers were female. Ninety percent of caregivers were the participating child's biological mother. One caregiver $(3.33 \%)$ was the child's aunt, one caregiver (3.33\%) was the child's maternal grandmother, and one (3.33\%) was the child's biological father. An independent samples $t$-test indicated that caregivers in the PCIT group were significantly older than caregivers in the UC group, $t=2.67, p=.01$. At the first assessment, caregivers in the PCIT group were 31 years, 10 months old $(S D=4.79$, range 23 years to 40 years). At the first assessment, caregivers in the UC group were 27 years 4 months old $(S D=2.78$, range 23 years to 31 years $)$. Ninety-seven percent of caregivers reported that they were Caucasian, and 3\% reported that they were African American. None of the caregivers reported that their ethnicity was "Latino/ Hispanic."

An average of $4.53(S D=1.63$, range $2-8)$ individuals lived in the child's home. Participating children had an average of 1.51 siblings $(S D=1.24$, range $0-5)$. Additionally, an average of $.45(S D=1.02$, range $0-4)$ extended family members lived in the home. Caregivers reported their marital status as follows: $37 \%$ married, $23 \%$ never married, $20 \%$ living with their partner, $13 \%$ divorced, and 7\% separated. A chi-square analysis indicated no significant difference between groups with regard to marital status, $X^{2}(4)=3.04, p=.55$. Five parents in the PCIT group and four parents in the UC group were married, and four parents in the PCIT group and two in the UC group were living with a partner. Four parents in the PCIT group and two parents in the UC group were separated or divorced. Two parents in the PCIT group and five parents in the UC group had never been married.

With regard to employment status, $13 \%$ of caregivers reported that they worked full time, $13 \%$ reportedly worked part time, $30 \%$ reported that they were unemployed and not seeking employment (e.g., homemaker), 23\% reported that they were unemployed and seeking employment, $13 \%$ reported that they were supported by Supplemental Social Security Income 
(SSI) or "Disability," 7\% reported that they were students, and 3\% reported that they were retired. The median reported household income was $\$ 20,800$ (range $\$ 8,016$ to $\$ 70,000$ ). Parents reported that their highest level of education was high school diploma or equivalent (33\%), some college (27\%), an associate's degree (23\%), some high school $(10 \%)$, less than $9^{\text {th }}$ grade $(3 \%)$, and a bachelor's degree (3\%). No significant differences were found between groups with regard to employment status, $X^{2}(6)=10.00, p=.13$, highest level of education, $X^{2}(5)=3.38, p=.64$, or annual household income, $t(24)=.80, p=.43$.

Therapists provided diagnostic impressions for 22 caregivers. Overall, therapists indicated that $60 \%$ of the caregivers had symptoms of a psychological disorder, $13 \%$ did not have symptoms of a psychological disorder, and it is unknown whether $27 \%$ of the sample had symptoms of a psychological disorder. More specifically, therapist impressions of caregivers indicated that four caregivers (13\%) were "anxious," two caregivers (7\%) had a trauma history, three caregivers (9\%) had symptoms of depression, three caregivers (9\%) had Bipolar Disorder, two caregivers (7\%) had Borderline Personality Disorder, one caregiver (3\%) had below average intelligence, and one caregiver (3\%) had a history of at least one psychotic episode. The percentages for the diagnostic impressions are not mutually exclusive. Fifty-three percent of caregivers reported that they had received therapy or counseling services in the past, and $10 \%$ chose to not answer this question. Significantly more caregivers in the PCIT group reported having received counseling, $X^{2}(1)=4.49, p=.03$. Eleven caregivers in the PCIT group and five caregivers in the UC group reported to have received counseling in the past. One caregiver in the PCIT group and two caregivers in the UC group did not answer this question.

Twenty-three percent of caregivers reported that they had been involved with Child Protective Services (CPS) in the past or present. Therapists reported that $33 \%$ of families had either current or past involvement with CPS. A chi-square analysis indicated no significant group difference with regard to current or past involvement in CPS, $X^{2}=.73, p=.39$. Six families in 
the PCIT group and four families in the UC group had current or past involvement with CPS, based on therapist report.

In summary, the groups were relatively equivalent with regard to child diagnoses, child age, parent sex, race, ethnicity, caregiver level of education, caregiver employment status, annual household income, and either current or past involvement in CPS. Significantly more male children were in UC, and caregivers in the PCIT group were significantly older. Significantly more caregivers in the PCIT group reported having received counseling services either currently or in the past. Although it did not reach statistical significance, many more children in the UC group were prescribed medication than in the PCIT group.

\section{Reliability of Observational Data}

Twenty-two percent of observations for Child-Directed Interaction were double-coded and coder for reliability was then assessed using the kappa statistic. Videotapes of observations were transcribed and each statement in the transcription was coded while viewing the videotape. Cohen's kappa was calculated for the Positive Skills Composite (i.e., labeled praise, reflections, behavior descriptions) and Negative Skills Composite (i.e., commands, questions, negative talk). The average kappa for the Positive Skills Composite was .83 (range .74 - 1.0), and the average kappa for the Negative Skills Composite was .86 (range .67- .94). For four of the cases, the Positive Skills Composite could not be calculated because both raters agreed that none of the skills of the Positive Skills Composite had occurred. For one case, the Negative Skills Composite could not be calculated because both raters agreed that none of the skills of the Negative Skills Composite had occurred.

Parent-Led Play and Clean up were combined for coding. Thirty-six percent of ParentLed Play and the Clean Up observation were double-coded for reliability using the kappa statistic. Cohen's kappa was calculated for Direct Commands, Indirect Commands, Child Compliance, and Labeled Praise for Compliance. The average kappa for Direct Commands was .92 (range .82 1.0). The average kappa for Indirect Commands was .84 (range .71-1.0). The average kappa 
statistic for Child Compliance was .82 (range .57-1.0). For 18 of 21 cases, a kappa statistic for Labeled Praise for Compliance could not be calculated because both raters agreed that none had occurred. For the three cases in which Labeled Praise for Compliance was present, the average kappa statistic was 1.0.

\section{Exploratory Questions}

\section{Description of treatment.}

$\boldsymbol{U C}$. The treatment modality at the first assessment for 14 families was BHRS (Behavioral Health Rehabilitative Services, "Wrap around"), and the treatment modality at the first assessment for one family was FBMHS (Family-Based Mental Health Services). Further analyses were conducted with and without the inclusion of the family who had received FBMHS, and no differences were found. Thus, all subsequent analyses included all UC families in the analyses. A difference in service delivery for the family in FBMHS was that they received services for a 3-month plan of care, and the goals for FBMHS were met. No other services were initiated for the participating child. One family in the study received no hours of BHRS. After the evaluator recommended BHRS, the insurance company disputed the medical necessity of BHRS for this child and peer review recommended outpatient therapy. The family did not initiate outpatient therapy.

Six therapists provided descriptions of treatment for 11 participants. Additionally, the number and type of hours billed between the assessments were reported for 12 participants. On average, children received $60.19(S D=85.02$, range $0-299.75)$ hours of billable Behavior Specialist Consultant, $119.36(S D=111.05$, range $0-290.25)$ hours of billable Therapeutic Support Staff, and an average of $1.25(S D=3.49$, range $0-12)$ hours of billable Mobile Therapist between assessments. About half of the Behavior Specialist Consultant's hours were typically spent in direct service with the child and the other half was spent in indirect services (e.g., reviewing data, treatment planning). It was common for a family to interact with the Behavior Specialist Consultant for about 60 to 75 minutes per week, per Behavior Specialist 
Consultant and participant report. Based on therapist report, participants had an average of 2.22 $(S D=1.30$, range 1-5) different Behavior Specialist Consultants and $1.67(S D=1.12$, range $1-$ 4) Therapeutic Support Staff "workers" during the study. Of the participants who had a Mobile Therapist, they had one Mobile Therapist during the course of the study. Thus, therapists reported that participants in the UC group received median total of $113.5(M=180.79, S D=$ 158.29, range $0-415.75$ ) hours of billable service during the study. Refer to Table 4 for a list of dose by participant. BHRS is paid for by Medical Assistance insurance. Behavior Specialist Consultant services reportedly are billed at $\$ 49$ to $\$ 52$ per hour, Mobile Therapy services reportedly are billed at $\$ 36$ to $\$ 49$ per hour, and Therapeutic Support Staff services reportedly are billed at $\$ 30$ per hour. Thus, it is estimated that insurance paid between $\$ 4,769.41$ and $\$ 6,760.93$, on average, for each family, based on average hours of each service type.

For a description of BHRS, refer to Table 5. Behavior Specialist Consultants reported that a member of the treatment team (e.g., Behavior Specialist Consultant, Therapeutic Support Staff, Mobile Therapist) delivered the following components of treatment during a "typical" week. Behavior Specialist Consultants reported spending about 9.25 minutes $(S D=20.21$, range 0-90) talking with the caregiver individually. Behavior Specialist Consultants reportedly spent 77.35 minutes $(S D=49.9$, range 5-195) with the caregivers and child together. Behavior Specialist Consultants reported that they typically spent about 18.75 minutes $(S D=32.83$, range 0 -95) working with the child individually. This time reflects the Behavior Specialist Consultants time with the family. The Therapeutic Support Staff spent time with the child although other family members may have been present in the vicinity.

During the time with the Behavior Specialist Consultant, $81 \%$ of parents were provided in-vivo feedback on behavior management strategies and/ or parent-child interactions during a "typical week." The frequency and quality of this feedback remains unknown. Child behavior was coded for $43 \%$ of families using operationally defined categories (e.g., noncompliance, aggression) during a "typical week," and parent behavior was coded using operationally defined 
behavior categories in $10 \%$ of the families during a "typical week." These observational data were presented to $4.8 \%$ of families on a weekly basis. Sixteen percent of families received weekly homework, and 5\% of families had their homework reviewed by a member of the treatment team. Therapeutic Support Staff implemented interventions with the child, modeled interventions for the caregivers, and collected observational data. No families were given a standardized parent-report measure on a weekly basis. However, parents completed a standardized parent-report measure approximately every 4 to 6 months when the medical necessity of BHRS for the child was re-evaluated. Additionally, observational data were almost always presented during these re-evaluations. See Table 5 for a summary of the treatment components.

Commonly reported treatment components included modeling of behavior management strategies and "positive" consequences. Strategies reportedly included using "if- then" statements, verbal countdown, rotating preferred and non-preferred activities, and planned ignoring. Improving skills deficits was also frequently identified as part of treatment.

PCIT. Six therapists returned the summary questionnaire and reported on attendance for 13 cases. Based on this measure, participants in PCIT received an average of 9.23 PCIT sessions between their two assessment points $(S D=5.73$, range $1-17)$. The average show rate was $60.03 \%(S D=.25$, range $.25-1.0)$. Two participants in the PCIT group transferred to BHRS during PDI, and two participants received BHRS in addition to PCIT throughout the course of the study. Additionally, two families transferred to non-PCIT outpatient therapy. Overall, families in the PCIT group received a median of 17 total hours of service $(M=80.83, S D=104.45$, range 1 to 294). Medical Assistance insurance reportedly pays $\$ 166$ per hour for outpatient therapy, including PCIT. Thus, it is estimated that insurance paid about $\$ 1,532.18$ for PCIT for each family, and $\$ 2,822$ for each family in the PCIT group during the study, based on median hours of therapy. 
Based on parent report, it was estimated that the participants in BHRS received an average of $59.00(S D=18.41$, range $32-60)$ Behavior Specialist Consultant hours and an average of $149.50(S D=35.65$, range 120-210) Therapeutic Support Staff hours. The researcher was unable to receive a therapist report of BHRS hours. Two participants transferred to other outpatient therapy services. Based on parent report and the average show rate, it was estimated that these families received an average of 8.5 (range $5-12$ ) other outpatient therapy sessions. Refer to Table 4 for a list of dose by participant.

Treatment integrity was $\mathrm{e}^{\mathrm{x}}$ amined separately for "CDI Teach," "CDI Coach," "PDI Teach," and "PDI Coach." Two CDI Teach sessions were reported. The average length of the session was 55 minutes (range $50-60$ ). The therapist gave the ECBI at one of these sessions $(50 \%)$, and the ECBI was not reviewed in either session ( $0 \%)$. One therapist collected homework at the CDI Teach session (50\%). One therapist coded the parent's CDI skills (50\%) and coached the parent in one CDI Teach for 10 minutes (50\%). The therapists reported talking to parents for an average of 32.5 minutes (range 15-50). It appears that one therapist used the hour primarily as a didactic session, and the other therapist provided a 15 -minute didactic with a 5-minute coding and 10-minute coaching session. Overall treatment integrity is not reported because only two CDI Teach sessions were reported, and the therapists used different approaches.

Therapists reported integrity for 31 CDI Coach sessions. Session duration was typically 56.96 minutes $(S D=4.97$, range $45-60)$. Therapists reported that they spent about 20.32 minutes $(S D=9.99$; range $0-40)$ talking with the parent during the session. The ECBI was given at $74.2 \%$ of the sessions, and it was reviewed at $32.3 \%$ of sessions. Homework was provided at $93.5 \%$ of sessions, and it was reviewed during $70 \%$ of sessions. Therapists coded using the DPICS in $93.5 \%$ of sessions, and they shared the coding data with the parent in $45.2 \%$ of sessions. They reported that they coached for 28.23 minutes $(S D=9.88$; range $10-50)$ and coached from behind a mirror during $96.8 \%$ of sessions. Therapists reported that only the caregiver-child dyad was present in the therapy room during coaching for $100 \%$ of cases. The 
therapists reportedly spent individual time with the child in $3.2 \%$ of sessions. Lastly, therapists reported that they looked at the PCIT manual before or during the session for $9.7 \%$ of sessions. Overall treatment integrity for CDI Coach sessions was $76.09 \%$.

Treatment integrity data were collected for seven PDI Teach sessions. The average length of the session was 56.67 minutes $(S D=5.16$, range $50-60)$. The therapist gave the ECBI at $85.7 \%$ of these sessions, and the ECBI was reviewed at $42.9 \%$ of sessions. Therapists collected and reviewed homework at $42.9 \%$ of sessions. Parent skills were coded in $28.6 \%$ of sessions and this coding occurred from behind a mirror. Parent observational data were presented and reviewed in $57.1 \%$ of sessions. The therapists reported talking to parents for an average of 47.14 minutes $(S D=21.96$, range $15-60)$. The therapist spent time alone with the child in $0 \%$ of the sessions. Homework was assigned in $100 \%$ of sessions. Overall treatment integrity for the seven PDI Teach sessions was 65.72\%.

Therapists reported integrity for 22 PDI Coach sessions. Session durations were typically 62.92 minutes $(S D=17.59$, range $50-120)$. Therapists reported that they spent about 23.41 minutes $(S D=7.62$, range $10-40)$ talking with the parent during the session. The ECBI was given at $95.5 \%$ of the sessions, and it was reviewed with the parent at $22.7 \%$ of sessions. Homework was provided at $100 \%$ of sessions, and it was reviewed during $77.3 \%$ of sessions. Therapists coded using the DPICS in $68.2 \%$ of sessions, and they shared the coding data with the parent in $9.1 \%$ of sessions. They reported that they coached for 35.86 minutes $(S D=20.66$, range $10-100$ ) and coached from behind a mirror during 100\% of sessions. Therapists reported that one sibling was present for coaching during $13.64 \%$ of sessions. The therapists reportedly spent individual time with the child in $0 \%$ of sessions. Lastly, therapists reported that they looked at the PCIT manual before or during the session for $36.4 \%$ of sessions. Overall treatment integrity for PDI Coach sessions was $71.60 \%$.

Overall treatment integrity for coaching sessions was $73.85 \%$. Therapists had the highest integrity to the model with coaching for the recommended amount of time (100\%), giving 
homework (96.75\%), administering the ECBI (84.85\%), and coding parent-child interactions (80.85\%). Aspects with the lowest integrity to the model included reviewing changes on the ECBI with the parent $(27.50 \%)$ and reviewing coding of parent and child behavior with the parent (27.15\%).

Differences between PCIT and UC. Chi-Square analyses indicated that PCIT and UC differed in many aspects. Caregivers in the PCIT group were significantly more likely to receive homework, $X^{2}=60.62, p<.0001$. Therapists in the PCIT group were significant more likely to review homework, $X^{2}=39.83, p<.0001$. Therapists in the PCIT group were significantly more likely to administer a standardized parent-report measure on a weekly basis, $X^{2}=42.97, p<$ .0001 , and more likely to review changes on the parent-report measures on a weekly basis, $X^{2}=$ $16.82, p<.0001$. PCIT therapists were significantly more likely to collect observational data of child behavior, $X^{2}=6.99, p=.008$, and parent behavior, $X^{2}=27.57, p<.001$. They were significantly more likely to review changes in observational data with the parent, $X^{2}=9.48, p=$ .009. UC therapists were asked if they provided "in-vivo" feedback to caregivers about behavior management strategies or interactions, which was similar to "coaching." PCIT therapists were significantly more likely than UC therapists to coach parents in behavior management strategies during parent-child interactions, $X^{2}=6.99, p=.008$. Lastly, children in the UC group had a significantly larger dose of treatment, $t(15)=-7.08, p<$ p.0001. Thus, PCIT and UC differed significantly in almost all aspects of treatment.

Treatment completion and attrition. Refer to Table 4 for a list of outcomes by participant. A chi-square analysis revealed no statistically significant differences between group with regard to completion and attrition, $X^{2}=7.55, p=.11$. In the PCIT group, two families (13\%) were still receiving PCIT, three families (20\%) had completed PCIT and were not receiving other therapeutic services, four families $(27 \%)$ had switched to another treatment, two families (13\%) had discontinued PCIT and were continuing with BHRS, and four families (27\%) had dropped out of PCIT and were not receiving other therapeutic services. Of those who dropped out of 
PCIT, three dropped out during CDI and one dropped out during PDI, based on therapist report. For those who received an alternative treatment, one switched during CDI, three switched during PDI. Of those who switched from PCIT to another treatment, two switched because their child received a diagnosis of an $\mathrm{ASD}$, one switched to an outpatient therapist closer to their home, and one began receiving primary medication management with therapy once per month. The two participants who received BHRS throughout the course of the study continued receiving BHRS but discontinued PCIT. Overall, eight of the 15 (53\%) families were still receiving some form of therapy services.

In the UC group, 7 families (78\%) were still receiving BHRS, four families had dropped out or been discharged due to poor compliance with prescribed hours $(27 \%)$, two families $(13 \%)$ had completed services and were not participating in other therapeutic services, one family $(7 \%)$ had switched to outpatient therapy twice per month, and one family (7\%) had never initiated BHRS after their initial evaluation. Additionally, in one family (7\%) the child had been removed from the home and placed in foster care because of caregiver maltreatment. Overall, eight of 15 (53\%) of families were still receiving some form of therapy services.

A descriptive difference is that only one family in UC switched from BHRS to outpatient therapy while six families in PCIT switched to BHRS. In the cases where the child was diagnosed with ASD, the therapist and family agreed that the family should transfer to BHRS. It should be noted that these two families had received some sessions of PDI. In the two cases where the families had received BHRS in addition to PCIT, the caregivers had made the decision to discontinue PCIT. Both families reported that the child did not engage in disruptive behavior in the clinic; however, the child continued to engage in disruptive behavior in the home.

\section{Social validity.}

Family satisfaction. Caregivers in both groups reported moderate satisfaction on the Therapy Attitude Inventory (Brestan, Jacobs, Rayfield, \& Eyberg, 1999). No significant difference was found between groups on overall caregiver satisfaction, $t=.18, p=.86$. On a 
scale from zero to 50 where zero indicates complete dissatisfaction and 50 indicates complete satisfaction, PCIT caregivers reported an average score of $39.33(S D=6.96$; range 26 - 49), and UC caregivers reported an average score of $38.77(S D=8.27$; range $26-50)$.

In qualitative responses about therapy, PCIT caregivers reported that they liked that they understood their child's behavior better because of therapy, and they liked the skills/techniques they learned to address child behavior and discipline. Caregivers reported that the most useful aspects were learning play skills and spending Special Play Time with their child almost daily, "connecting" with their child more, time-out, and the decrease in annoying vocals (e.g., whining, screaming). Caregivers disliked how long it took to move from CDI to PDI.

Caregivers in the UC group liked the support they received, more understanding of what helps their child, that their child learned skills, and that the services were one-on-one with the child. Useful aspects of treatments were "positive reinforcement," increased child communication, and talking about the child's feelings. Caregivers did not like the changes in staff, how many hours staff spent in their homes, staff giving in or not managing the child's behavior, and not receiving new ideas on how to address child behavior. More than one parent reported the themes presented here. To see all responses and caregivers' individual responses, refer to Table 6 .

Acceptability of PCIT for therapists. Six therapists responded to the survey. Four were therapists who had referred families to the study, and two of the therapists had not referred families to the study. They reported to have completed 4.5 cases (range $2-6$ ). They reportedly had "attempted" PCIT with about 18.75 families (range 6 - 35). They were using PCIT with four families (range $0-11$ ) at the time that they completed the survey. Each therapist provided different perspectives with little convergence. A few main themes were reported here, but refer to Table 7 for complete results. The therapists reported that they liked the structure, the datadriven approach, and working with the parents to change their own behavior. Aspects that they did not like were technical difficulties with the bug-in-ear equipment, that PCIT does not address 
emotions, and limited time to discuss family concerns. They found the parent skills, coaching, and weekly homework review to be useful. All therapists planned to continue using PCIT and would recommend that others receive training.

The therapists identified some difficulties and barriers. Therapists reported concerns with maintenance of treatment gains after therapy was completed. For families who were receiving PCIT and BHRS at the same time, two therapists found the strategies used to be inconsistent across services and confusing for parents. While some therapists' clients were able to receive BHRS in addition to PCIT, another therapist reported frustration that she could not provide PCIT to families who were in BHRS in her county because of restriction from the insurance company. Other challenges included generalization of skills to the home, sustained parental engagement, and coaching PDI initially. Transportation could be a barrier for some families.

Therapists reported adaptations and modification in terms of what they changed, added, and left out. Therapists made some modifications to PCIT for cases when the child had experienced trauma by taking a "break" from PCIT or integrating some other therapeutic strategies with the child when needed. A therapist also had made modifications when the caregiver could not physically maneuver the child. Two therapists reported that they used almost all components of the treatment, and one reportedly always included homework review, coding, and the ECBI. Two therapists did not always use the aspects of PCIT for addressing public behavior, and one had difficulty with keeping parents engaged in therapy during PDI. One therapist did not always provide the ECBI and did not find it useful to give at every session.

\section{Objective Treatment Outcomes}

ECBI. Refer to Table 4 for a list of ECBI scores at the first and second assessment by participant. A 2 (Condition: PCIT, UC) X 2 (Time: Assessment 1, Assessment 2) Analysis of Variance was conducted to evaluate the effect of treatment and time on parent report of the Intensity Scale of the ECBI, a parent report of child disruptive behavior during the previous week. The data were examined to determine if the assumptions of the RM ANOVA had been 
met. The assumption of sphericity was violated, and a Greenhouse-Geisser correction was used. The interaction of Condition $\mathrm{X}$ Time was not significant, $F(1,23)=2.4, p=.132$, MSE $=$ 649.46. The main effect of Time was significant, $F(1,23)=8.26, p=.009$, MSE $=649.46$. The main effect of Condition was not significant, $F(1,23)=.56, p=.46, \mathrm{MSE}=3241.19$. Thus, all participants, regardless of condition, showed statistically significant improvement over time. For means and standard deviations, refer to Table 8 .

Effect size using Cohen's $d$ was calculated for the change from the first to the second assessment in each group and for between groups at the second assessment. A large effect was found for the PCIT group from the first to the second assessment, $d=.74$. A small effect was found for the UC group from the first to the second assessment, $d=.31$. Lastly, a medium effect was found between the conditions at the second assessment, $d=.53$, with the PCIT group demonstrating greater changes on the ECBI.

At the first assessment, $70 \%$ of caregivers indicated clinically significant levels of child disruptive behavior in the week prior to the assessment. A chi-square analysis indicated no statistically significant difference between the groups with regard to the number of caregivers in each group who reported clinically significant disruptive behavior, $X^{2}=1.43, p=.23$, at the first assessment. At the second assessment, $44 \%$ of caregivers indicated clinically significant levels of child disruptive behavior in the week prior to the assessment. A chi-square analysis approached statistical significance with regard to the number of caregivers in each group who reported clinically significant disruptive behavior at the second assessment, $X^{2}=3.38, p=.07$.

Twenty-five percent of caregivers in the PCIT group reported clinically significant disruptive behavior at the second assessment, and $62 \%$ of the UC group reported clinically significant disruptive behavior at the second assessment. For caregivers in the PCIT group, 33\% never reported disruptive behavior in the clinically significant range, $25 \%$ reported clinically significant disruptive behavior at both time points, and $42 \%$ reported a decrease in child disruptive behavior from the clinically significant to the normative range. For caregivers in the 
UC group, $23 \%$ never reported disruptive behavior in the clinically significant range, $62 \%$ reported clinically significant disruptive behavior at both time points, and $15 \%$ reported a decrease in child disruptive behavior from the clinically significant to the normative range. No caregivers in either group reported that the child's disruptive behavior increased from the normative range to the clinically significant range during the study. A chi-square analysis indicated the change in clinical significance whether increasing, decreasing, or remaining the same on the Intensity Scale of the ECBI was not statistically significant, $X^{2}=3.67, p=.16$.

CBCL. A 2 (Condition: PCIT, UC) X 2 (Time: Assessment 1, Assessment 2) Analysis of Variance was conducted to evaluate the effect of treatment and time on parent report on the Externalizing Scale of the CBCL. The data were examined to determine if the assumptions of the RM ANOVA had been met. The assumption of sphericity was violated, and a GreenhouseGeisser correction was used. The interaction of Condition X Time was not significant, $F(1,23)$ $=1.40, p=.25, \mathrm{MSE}=45.24$. The main effect of Time was significant, $F(1,23)=7.60, p=.01$, $\mathrm{MSE}=45.24$. The main effect of Condition was not significant, $F(1,23)=.17, p=.69, \mathrm{MSE}=$ 238.23. Thus, all participants, regardless of condition, showed statistically significant improvement in parent-reported externalizing behavior over time. For means and standard deviations, refer to Table 8 .

Effect size using Cohen's $d$ was calculated for the change from the first to the second assessment in each group and for between groups at the second assessment. A medium effect was found for the PCIT group from the first to the second assessment, $d=.64$. A small effect was found for the UC group from the first to the second assessment, $d=.25$. Lastly, a small effect was found between the conditions at the second assessment, $d=.34$, with the PCIT group demonstrating greater changes on the CBCL Externalizing Scale.

At the first assessment, $67 \%$ of caregivers reported clinically significant Externalizing Behavior on the CBCL. At the second assessment, $44 \%$ of caregivers reported clinically significant Externalizing Behavior on the CBCL. A chi-square analysis indicated no statistically 
significant difference between groups with regard to clinically significant externalizing scores at the first time point, $X^{2}=1.22, p=.27$, or the second time point, $X^{2}=1.07, p=.30$. For caregivers in the PCIT group, 50\% never reported externalizing behavior in the clinically significant range, $33 \%$ reported externalizing behavior in the clinical range at both time points, $17 \%$ reported a decrease in externalizing behavior from the clinical to the normative range, and $0 \%$ reported that externalizing behavior moved into the clinical range from the normative range. For caregivers in the UC group, $31 \%$ never reported externalizing behavior in the clinically significant range, $46 \%$ reported externalizing behavior in the clinical range at both time points, $15 \%$ reported a decrease in externalizing behavior from the clinical to the normative range, and $8 \%$ reported that externalizing behavior moved into the clinical range from the normative range. A chi-square analysis indicated the change in clinical significance on the Externalizing Score of the CBCL was not statistically significant, $X^{2}=1.76, p=.62$.

A 2 (Condition: PCIT, UC) X 2 (Time: Assessment 1, Assessment 2) Analysis of Variance was conducted to evaluate the effect of treatment and time on parent report on the Internalizing Scale of the CBCL. The data were examined to determine if the assumptions of the RM ANOVA had been met. The assumption of sphericity was violated, and a GreenhouseGeisser correction was used. The interaction of Condition X Time was not significant, $F(1,23)$ $=.00, p=1.0, \mathrm{MSE}=43.75$. The main effect of Time was not significant, $F(1,23)=1.43, p=$ $.24, \mathrm{MSE}=43.75$. The main effect of Condition was not significant, $F(1,23)=1.56, p=.24$, MSE $=129.67$. Thus, none of the participants, regardless of condition, showed statistically significant improvement in parent-reported internalizing behavior over time. For means and standard deviations, refer to Table 8 .

Effect size using Cohen's $d$ was calculated for the change from the first to the second assessment in each group and for between groups at the second assessment. A small effect was found for the PCIT group from the first to the second assessment, $d=.28$. A small effect was found for the UC group from the first to the second assessment, $d=.22$. Lastly, a small effect 
was found between the conditions at the second assessment, $d=.35$, with the PCIT group having less internalizing child behavior.

At the first assessment, $30 \%$ of caregivers reported clinically significant child internalizing behavior. A chi-square analysis revealed a clinically significant difference between condition in the Internalizing Score at the first assessment, $X^{2}=3.96, p=.05$. Specifically, $47 \%$ of the UC group had clinically significant internalizing scores on the CBCL compared to $13 \%$ of the PCIT group at the first assessment. At the second assessment, $20 \%$ of caregivers reported clinically significant child internalizing behavior. A chi-square analysis did not indicate a clinically significant difference between conditions in the Internalizing Score at the second assessment, $X^{2}=1.96, p=.16$. For caregivers in the PCIT group, $83 \%$ never reported internalizing behavior in the clinically significant range, $0 \%$ reported internalizing behavior in the clinical range at both time points, $8 \%$ reported a decrease in internalizing behavior from the clinical to the normative range, and $8 \%$ reported that internalizing behavior moved into the clinical range from the normative range. For caregivers in the UC group, 54\% never reported internalizing behavior in the clinically significant range, $23 \%$ reported internalizing behavior in the clinical range at both time points, $15 \%$ reported a decrease in internalizing behavior from the clinical to the normative range, and $8 \%$ reported that internalizing behavior moved into the clinical range from the normative range. A chi-square analysis indicated the change in clinical significance on the Internalizing Score of the CBCL was not statistically significant, $X^{2}=3.83, p=$ .28 .

\section{Observation of parent-child interactions.}

Child led play. The Positive Parent Composite (labeled praise, reflections, behavior desriptions) was examined. A 2 (Condition: PCIT, UC) X 2 (Time: Assessment 1, Assessment 2) Analysis of Variance was conducted to evaluate the effect of treatment and time on the observed Positive Parenting Composite. The data were examined to determine if the assumptions of the RM ANOVA had been met. The Positive Parent Composite was skewed at both assessment 
points because few caregivers used the verbal skills. The Positive Parent Composite was log transformed to create a normal distribution. Additionally, the assumption of sphericity was violated, and a Greenhouse-Geisser correction was used. The interaction of Condition X Time was not significant, $F(1,23)=.13, p=.72, \mathrm{MSE}=.11$. The main effect of Time was not significant, $F(1,23)=.07, p=.80, \mathrm{MSE}=.11$. The main effect of Condition was significant, $F$ $(1,23)=12.24, p=.002, \mathrm{MSE}=.22$. Thus, participants in the PCIT group had a statistically significantly higher Positive Parenting Composite at both assessment times. For means and standard deviations, refer to Table 8.

Effect size using Cohen's $d$ was calculated for the change from the first to the second assessment in each group and for between groups at the second assessment. A small effect was found for the PCIT group from the first to the second assessment, $d=.13$, on the Positive Parent Composite. A small effect was found for the UC group from the first to the second assessment, $d$ $=-.16$, on the Positive Parent Composite. Lastly, a large effect was found between the conditions at the second assessment, $d=1.20$, on the Positive Parent Composite with the PCIT group having a larger Positive Parent Composite.

The Negative Parenting Composite (commands, questions, negative talk) was examined. A 2 (Condition: PCIT, UC) X 2 (Time: Assessment 1, Assessment 2) Analysis of Variance was conducted to evaluate the effect of treatment and time on the observed Negative Parenting Composite. The data were examined to determine if the assumptions of the RM ANOVA had been met. The assumption of sphericity was violated, and a Greenhouse-Geisser correction was used. The interaction of Condition X Time was not significant, $F(1,23)=.07, p=.79$, MSE $=$ 78.80. The main effect of Time was not significant, $F(1,23)=1.60, p=.22$, MSE $=78.80$. The main effect of Condition was significant, $F(1,23)=5.37, p=.03$, MSE $=195.77$. Thus, the parents in the PCIT group had a significantly smaller Negative Parenting Composite at both time points. For means and standard deviations, refer to Table 8. 
Effect size using Cohen's $d$ was calculated for the change from the first to the second assessment in each group and for between groups at the second assessment. A small effect was found for the PCIT group from the first to the second assessment, $d=.23$, for the Negative Parenting Composite. A small effect was found for the UC group from the first to the second assessment, $d=.31$, for the Negative Parenting Composite. Lastly, a moderately large effect was found between the conditions at the second assessment, $d=.70$, for the Negative Parenting Composite with the PCIT group having a smaller Negative Parenting Composite.

Parent led play and clean up. Refer to Table 4 for a list of child compliance at the first and second assessment by participant. Child Compliance (observed child compliance divided by total commands) was examined. A 2 (Condition: PCIT, UC) X 2 (Time: Assessment 1, Assessment 2) Analysis of Variance was conducted to evaluate the effect of treatment and time on the observed child compliance. The data were examined to determine if the assumptions of the RM ANOVA had been met. The assumption of sphericity was violated, and a GreenhouseGeisser correction was used. The interaction of Condition X Time approached significance, $F$ (1, $23)=3.98, p=.06, \mathrm{MSE}=.03$. The main effect of Time was significant, $F(1,23)=12.24, p=$ $.002, \mathrm{MSE}=.03$. The main effect of Condition was not significant, $F(1,23)=.62, p=.44$, MSE $=.06$. For means and standard deviations, refer to Table 8 .

Effect size using Cohen's $d$ was calculated for the change from the first to the second assessment in each group and for between groups at the second assessment. A large effect was found for the PCIT group from the first to the second assessment, $d=.93$, for child compliance. A medium effect was found for the UC group from the first to the second assessment, $d=.39$, for child compliance. Lastly, a moderately large effect was found between the conditions at the second assessment, $d=.69$, for child compliance with the PCIT having higher compliance.

The percentage of parental direct commands to total commands (direct commands divided by total commands) was examined. A 2 (Condition: PCIT, UC) X 2 (Time: Assessment 1, Assessment 2) Analysis of Variance was conducted to evaluate the effect of treatment and time 
on the observed percentage direct commands. The data were examined to determine if the assumptions of the RM ANOVA had been met. The assumption of sphericity was violated, and a Greenhouse-Geisser correction was used. The interaction of Condition X Time was not significant, $F(1,23)=3.31, p=.08, \mathrm{MSE}=.01$. The main effect of Time was significant, $F(1$, $23)=8.91, p=.007, \mathrm{MSE}=.01$. The main effect of Condition was significant, $F(1,23)=16.34$, $p=.001, \mathrm{MSE}=.03$. Thus, caregivers in the PCIT group did not have a change in the percentage of direct commands over and above the change observed in the UC group. For means and standard deviations, refer to Table 8 .

Effect size using Cohen's $d$ was calculated for the change from the first to the second assessment in each group and for between groups at the second assessment. A large effect was found for the PCIT group from the first to the second assessment, $d=-.83$, for the percentage of parental direct commands. A small effect was found for the UC group from the first to the second assessment, $d=-.31$, for the percentage of parental direct commands. Lastly, a medium effect was found between the conditions at the second assessment, $d=.64$, for the percentage of parental direct commands with the PCIT group having a smaller percentage of direct commands to total commands.

Labeled praise following compliance was examined. The assumptions of the RM ANOVA were examined. The data for Labeled Praise for Compliance were skewed and could not be corrected using a transformation. The variable was recoded to indicate the presence or absence of Labeled Praise for Compliance. A chi-square analysis examined the differences between the groups at the first and second assessments. At the first assessment, a chi-square analysis indicated no significant difference between conditions (Condition: PCIT, UC) with regard to the presence of labeled praise for compliance, $X^{2}=1.03, p=.31$. At the second assessment, a chi-square analysis approached a significant difference between the conditions with regard to the presence of labeled praise for compliance, $X^{2}=3.69, p=.06$. At the second 
assessment, $25 \%$ of PCIT group provided praise for compliance compared to $0 \%$ of the UC group. For means and standard deviations, refer to Table 8.

Effect size using Cohen's $d$ was calculated for the change from the first to the second assessment in each group and for between groups at the second assessment. A medium effect was found for the PCIT group from the first to the second assessment, $d=.69$, for contingent labeled praise. A medium effect in the opposite direction was found for the UC group from the first to the second assessment, $d=-.40$, for contingent labeled praise. Lastly, a medium effect was found between the conditions at the second assessment, $d=.69$, for contingent labeled praise with the PCIT group having more contingent labeled praise.

Parenting stress. A 2 (Condition: PCIT, UC) X 2 (Time: Assessment 1, Assessment 2) Analysis of Variance was conducted to evaluate the effect of treatment and time on parent report on the PSI. The data were examined to determine if the assumptions of the RM ANOVA had been met. The assumption of sphericity was violated, and a Greenhouse-Geisser correction was used. The interaction of Condition X Time was not significant, $F(1,23)=.25, p=.62$, MSE $=$ 208.76. The main effect of Time was not significant, $F(1,23)=.55, p=.47, \mathrm{MSE}=208.76$. The main effect of Condition was not significant, $F(1,23)=.00, p=.999, \mathrm{MSE}=846.25$. Thus, neither group showed statistically significant improvement in parent-reported parenting stress over time. For means and standard deviations, refer to Table 8.

Effect size using Cohen's $d$ was calculated for the change from the first to the second assessment in each group and for between groups at the second assessment. A negligible effect was found for the PCIT group from the first to the second assessment, $d=.04$, for parenting stress. A small effect was found for the UC group from the first to the second assessment, $d=$ .23 , for parenting stress. Lastly, a negligible effect was found between the conditions at the second assessment, $d=.08$, for parenting stress.

At the first assessment, five caregivers (17\%) had defensive responding on the PSI, and at the second assessment, two caregivers (13\%) had defensive responding. Four caregivers (13\%) 
indicated clinically significant levels of parenting stress at the first assessment, and four caregivers $(16 \%)$ indicated clinically significant levels of parenting stress at the second assessment. A chi-square indicated no differences in clinical significance between groups at the

first time point, $X^{2}=.00, p=1.0$, or at the second time point, $X^{2}=.00, p=.93$, as both groups had two caregivers with clinically significant levels of parenting stress at both time points. For caregivers in the PCIT group, $75 \%$ never reported clinically significant parenting stress, $8 \%$ reported clinically significant parenting stress at both time points, $8 \%$ reported clinically significant improvement in parenting stress, and $8 \%$ reported a clinically significant increase in parenting stress. For caregivers in the UC group, $77 \%$ never reported clinically significant parenting stress, $0 \%$ reported clinically significant parenting stress at both time points, $8 \%$ reported a clinically significant improvement in parent stress, and 15\% reported a clinically significant increase in parenting stress. It should be noted that one of the caregivers in the UC group who reported clinically significant stress at the first time point did not participate in the second assessment. A chi-square analysis indicated no statistically significant differences between the groups with regard to change in clinical significance, $X^{2}=1.35, p=.72$.

\section{Supplemental Analyses}

Examination of dose and outcomes. As the dose of therapy in terms of hours of clinical service was significantly different between the groups, the relation between dose and outcomes was examined. The change on the ECBI as a function of the total number of hours of therapy was graphed using a scatterplot. The scatterplots indicated that the families who completed PCIT and the child who began receiving stimulant medication had the greatest changes. The PCIT families who had not yet completed, dropped out, or transferred to BHRS or other outpatient therapy had changes commensurate with those demonstrated in the UC group. Negative change occurs when the parents reported worse child behavior at the second assessment compared to the first assessment. Four families in the PCIT group, including two of the four who had received BHRS, had negative change on the ECBI. Seven families in the UC group had negative changes on the 
ECBI. See Figure 1 to view outcomes for all families, and see figure 2 for a scatterplot including only families who did not receive BHRS. The data show no relationship between increased service hours and greater improvements on the ECBI in either graph.

"Pure" PCIT and BHRS. Outcomes on standardized measures were examined for families who received only PCIT and for families who received only BHRS. These analyses were conducted because transfers to types of therapy were frequent. Participants who had not transferred to another type of therapy were included in the analyses. This yielded six families in the PCIT group and ten families in the BHRS group. These results should be interpreted with caution because the limited sample sizes produced a distribution that was not normal, the assumption of sphericity was not met, and the groups were not equivalent at the first assessment. Specifically, the PCIT group had a significant lower percentage of direct commands $(M=.65, S D$ $=.12)$ than the BHRS group $(M=.78, S D=.08)$ at the first assessment, $t(7.92)=-2.33, p=.05$. Significant differences at the first assessment were not noted for the CBCL Externalizing Score, $t$ $(14)=-.66, p=.52$, CBCL Internalizing Score, $t(14)=-1.52, p=.15$, ECBI, $t(14)=-.66, p=$ .52 , Positive Parenting Composite, $t(6.28)=1.54, p=.17$, Negative Parenting Composite, $t(14)$ $=-1.50, p=.16$, Child Compliance, $t(14)=.43, p=.68$. Thus, child behavior was not equivalent across the two groups at the first assessment.

Examination of the interaction using a 2 (first assessment, second assessment) X 2 (PCIT, BHRS) RM ANOVA revealed that families in the "pure" PCIT group had significantly greater decreases on the ECBI, $F(1,13)=6.03, p=.03, \mathrm{MSE}=394.69$, and increases in use of labeled praise following compliance, $X^{2}=3.81, p=.05$. No other significant differences were found for standardized outcome measures (see Table 10). Effect sizes were graphed across outcome measures (Figure 4), and a similar pattern was observed as with the whole sample. Effect sizes for child disruptive and externalizing behavior outcomes in the PCIT group ranged from 1.02 to 1.44. Effect sizes for child disruptive and externalizing behavior in the BHRS group ranged from -.01 to .71 . 


\section{Summary of Results}

Children in both groups made significant improvements in disruptive behavior as measured by caregiver report and direct observation. No significant differences between the groups on parent-reported or observed child behavior were present at the first assessment.

Additionally, a significant difference was not present in the number of children diagnosed with an ASD between the groups or the number of families involved with CPS either currently or in the past. Caregivers in the PCIT group reported moderately large effect sizes in child disruptive and aggressive behaviors from the first to second assessment. In comparison, caregivers in the UC group reported small effect sizes in child disruptive and aggressive behavior during the course of the study (see Figure 3). Large changes in child compliance were observed in the PCIT group while medium changes in child compliance were observed in the UC group (see Figure 3).

Seven families (58\%) in the PCIT group had improvements in both parent-reported and observed child behavior at the second assessment. An additional family (8\%) reported improvements in child behavior although change was not observed in compliance. An additional three families $(25 \%)$ had observed improvement in child compliance although parent report did not indicate improvement. One family $(8 \%)$ in the PCIT group did not have improvements in either parent-reported or observed child behavior. Three families $(23 \%)$ in the UC group had improvements in both parent-reported and observed child behavior at the second assessment. An additional four families (31\%) in the UC group had parent report improvements in child behavior although improvements were not observed in child compliance. An additional three families (23\%) had improvements in child compliance that were not corroborated by parent-reported improvements in child behavior. Three families (23\%) in the UC group did not have improvements in either parent-reported or observed child behavior. Refer to Figure 5 for comparison of improvement in the whole sample and figure 6 for comparison of improvement in the "pure" sample. 


\section{Discussion}

The current investigation examined the effectiveness of PCIT delivered by community therapists and the effectiveness of PCIT compared to UC in the community. PCIT showed promise as a cost-effective treatment modality for young children with disruptive behavior. PCIT produced reductions in child disruptive behavior that were at least as beneficial as UC, with effect sizes that were larger than UC. Parents who received PCIT were as satisfied with treatment as those who received UC. Yet, PCIT produced these outcomes with significantly fewer hours of service than UC. The results partially supported the hypotheses that children in the PCIT group would have greater improvements in disruptive behavior as measured by parent report and direct observation. Observation of parent behavior did not indicate changes in parent behavior in the hypothesized directions with the exception of labeled praise for compliance. The current study supported past examinations of PCIT in community settings that have found promising outcomes for child disruptive behavior (Abrahamse et al., 2012; Galanter et al., 2012; Lanier et al., 2011; Pearl et al., 2011; Phillips et al., 2008) and past efficacy research that has found favorable outcomes for both PCIT and UC (McCabe \& Yeh, 2009).

\section{Differences in Dose Between the Groups}

Families in the PCIT group received significantly fewer hours of direct service than those in the UC group; however, families in the PCIT group had larger treatment effects for child disruptive behavior than the caregivers in the UC group. The most cautious interpretation of the data suggests that the treatment groups were equivalent in treatment outcomes. Thus, a cautious interpretation of the data suggest that 10 sessions of PCIT, with 17 hours of therapy, was equivalent to about 113.5 billable hours of UC. Examination of effect sizes indicates larger effect sizes for PCIT in reported and observed child disruptive behavior. Therefore, it is possible that a relatively low number of PCIT service hours may be superior to far more hours of UC.

\section{Child Outcomes}


The medium to large effect sizes for reported and observed child disruptive behavior in the PCIT group from the first to second assessment indicate improvements in child behavior (see Table 9, Figure 3). In contrast, small effect sizes were found for the UC group across all measures of child behavior. A larger effect size for child compliance in the PCIT group was observed in the current study than in other efficacy studies of PCIT (see Table 9). However, only $25 \%$ of the PCIT group, and none of the UC group, displayed compliance at or above $75 \%$ (see Table 4), which is a goal of PDI (Eyberg \& Funderburk, 2011). For the three children who completed PCIT, parent report of child behavior was in the normative range and observed child compliance had improved. However, compliance in these families was not observed at or above 75\%. Ten families had improvements in both parent-report and observed child behavior (see Table 4), and seven of these families were in the PCIT group. Overall results indicate that PCIT produced better outcomes than UC, but few families achieved the goals for child compliance set by the PCIT manual (Eyberg \& Funderburk, 2011).

The compliance rate of the children in both groups at the first assessment was lower than past research in the clinic setting (e.g., McCabe \& Yeh, 2009; Schuhmann et al., 1998); however, it was similar to observed compliance at pretreatment in the home setting (e.g., Galanter et al., 2012). It is possible that child compliance is higher on average in the clinic than in the home. The large effect of PCIT on observed child compliance in the home setting and parent report of child behavior provides support that treatment gains generalize to the home setting. Additionally, although child compliance may be higher in the clinic than in the home, training compliance to above $75 \%$ in the clinic may result in noticeable changes in child behavior at home.

Small effects were reported for child internalizing symptoms in both groups. About one third of the sample had clinically significant internalizing symptoms at the first assessment. Significant changes in internalizing symptoms were not reported in either condition of the current study. The current study does not contribute to the literature suggesting that behavioral parent training results in reduction of child internalizing symptoms (Kaminski et al., 2008). 


\section{Parent Outcomes}

Observed behavior. Observation of parent behavior did not support hypotheses with the exception of labeled praise for compliance. Direct observation of parent behavior in both groups indicated little increase in the "PRIDE" skills (labeled praise, reflections, behavior descriptions), little decrease in the "Don't" Skills (commands, questions, negative talk), less use of direct commands in Parent Led Play and Clean Up, and the presence but infrequent use of labeled praise following compliance. Greater parental use of PCIT skills may have had an impact on child behavior that more closely matched the effect sizes of PCIT in other efficacy and effectiveness studies.

Contrary to hypothesized outcomes and previous literature (e.g., Galanter et al., 2012; McCabe \& Yeh, 2009), a large effect size from the first to second assessment was observed in the PCIT group in caregivers using fewer direct commands in relation to total commands. Interestingly, caregivers in the UC group displayed a percent of direct commands to total commands that was around the PCIT "mastery" criteria for commands at both time points. In contrast, PCIT caregivers regressed further from the PCIT "mastery" criteria for commands at the second assessment. Contingent labeled praise for compliance was only present in the PCIT group at the second assessment. However, it remained low frequency even in the PCIT group.

There are many possible explanations for the unexpected findings for observed parent behavior. Observed parent behavior may reflect that the first assessment was not a "true" pretreatment session, caregiver drift from the PCIT skills, poor parental integrity to the PCIT model at home in general, or an insufficient number of PCIT sessions to produce changes. Significant differences in CDI skills between groups at both assessments may suggest that caregivers were changing their play behavior within the first few weeks of PCIT. The unexpected increase in indirect commands and low frequency of labeled praise for compliance could reflect that families dropped out of PCIT before PDI or did not complete PDI. Although $83 \%$ had begun PDI by the second assessment, only $25 \%$ of PCIT families had completed PDI. It is also possible 
that parents switched from direct to indirect commands as child compliance improved and direct commands were less necessary. It could be argued that the observed parent behavior was sufficient to make measurable changes in child behavior as large effects were found with regard to observed child compliance in the PCIT group.

Parenting stress. Caregivers in neither group reported significant decreases in parenting stress. The hypothesis was not supported that caregivers in the PCIT group, compared to the UC group, would report a greater decrease in total parenting stress. However, only $13 \%$ of the original sample reported clinically significant parenting stress. A floor effect may have occurred with the variable of parenting stress as there was not room for improvement. Thus, a reduction of parenting stress may not have occurred because parenting stress was not a problem for the majority of the families in either group.

\section{Strengths of Current Study}

This study provides information regarding dissemination of PCIT, implementation of PCIT, and outcomes of PCIT in the "real world." Therapists had higher caseloads, fewer resources, and less supervision in the model than efficacy studies of PCIT. The researcher did not interfere with the selection of families who participated in PCIT or the delivery of PCIT in terms of integrity to the model. Almost no exclusion criteria were imposed for eligibility in the study. The therapists were not receiving ongoing consultation or supervision by a PCIT expert and fidelity to the model was not closely monitored. No feedback was given to the PCIT therapists regarding their integrity to the PCIT protocol. In efficacy studies (e.g., Schuhmann et al., 1998; McCabe \& Yeh, 2009; Nixon et al., 2002), almost all PCIT sessions were videotaped, which would allow for feedback on integrity to the PCIT model, delivery of therapy in general, accuracy of coding, and quality of coaching. Thus, the current study may be a better representation than efficacy studies as to how PCIT performs when community therapists are trained and subsequently implement PCIT in their community agencies. 
The current study has methodological strengths over previous effectiveness studies of PCIT. This is the first prospective examination of the effectiveness of PCIT delivered by community therapists as past PCIT effectiveness studies have been retrospective in that records were reviewed to examine treatment outcomes (e.g., Abrahamse et al., 2012; Galanter et al., 2012; Pearl et al., 2011; Phillips et al., 2008). Unlike other effectiveness studies of PCIT (e.g., Abrahamse et al., 2012; Galanter et al., 2012; Lanier et al., 2011; Pearl et al., 2011; Phillips et al., 2008), independent research assistants who had been trained to reliability in the observational coding system coded parent-child interactions, and the reliability of observational coding was examined. This is the first effectiveness study to present reliability data for the observational data. This is the third effectiveness study of PCIT to present the data from parent-report measures and observational data (see Galanter et al., 2012; Pearl et al., 2011), and it is the second effectiveness study of PCIT to present observational data from both CDI and PDI (see Galanter et al., 2012). Thus, this is one of the first studies to use a standardized, bimodal assessment with reliability of observational data to examine the effectiveness of community-delivered PCIT.

A distinguishing factor between the current study and other studies of PCIT is that an independent person without a clinical relationship with the family observed parent-child interactions in the home setting. The demand characteristics of the situation may have been lower than in the clinic or with the child's therapist present. The majority of observational data in the PCIT efficacy literature presents parent-child observations videotaped in the clinic and coded by research assistants who may have little, if any, clinical relationship with the family. Of the effectiveness studies of PCIT that have presented observational data, they present data coded by the child's therapist without reliability data. In the case of the effectiveness studies, the therapists' coding may be biased in favor of showing improvement. Thus, the current study contributes to the literature by examining videotaped parent-child observations in the home setting with independent and unbiased coders. 
This is the first effectiveness study to compare the use of PCIT and UC by community therapists. Prior examinations of the effectiveness of PCIT have used pre to post designs and all groups received PCIT (e.g., Abrahamse et al., 2012; Galanter et al., 2012; Lanier et al., 2011; Pearl et al., 2011; Phillips et al., 2008). This is the second research study to compare PCIT to another treatment. McCabe and Yeh (2009) was the first study to compare PCIT to UC, and that study is best characterized as an efficacy study that took place in a community clinic. For example, graduate students served as PCIT therapists, sessions were videotaped for reliability, and therapists received close supervision in the PCIT model. Thus, the current study increases knowledge regarding the effectiveness of PCIT compared to other treatments.

The current study provides information regarding the effectiveness of the dissemination of evidence-based practices for young children. Descriptive data were collected regarding how many therapists trained in PCIT continue to actively implement PCIT within the first two years of their training. This is the second study to examine treatment integrity in a sample of community PCIT therapists (see Travis \& Brestan-Knight, 2012). Lastly, the current study contributes to the knowledge of the effectiveness in the community setting of a probably efficacious treatment and the actual outcomes that can be expected when community therapists are trained in an evidencebased treatment. These are important goals in the development of evidence-based practices for children (Hoagwood et al., 1995; Weisz et al., 2005; Weisz et al., 2006).

The current study increases understanding of usual care in community settings and BHRS in Pennsylvania. Few previous studies have examined usual care in general or BHRS in Pennsylvania specifically (for exceptions see Garland et al., 2010; Knapp \& Reed, 2010; Toffalo, 2000). The description of usual care and outcomes of BHRS are relevant to the increasing emphasis on effective therapy in the community.

Lastly, the current study provides a preliminary examination of generalization of treatment gains to the home setting. In the case of the efficacy studies, the clinic setting may serve as a stimulus cue to the caregiver as to the behaviors expected, at least at the post treatment 
session. This is the second study to examine parent skills in the home when PCIT is delivered in the clinic (see Naik-Polan \& Budd, 2008). It is the first study to examine PDI parent skills in the home setting with clinic-based PCIT. Thus, observed parent-child interactions in the current study's PCIT group may reflect generalization of parenting skills from the clinic to the home setting.

\section{PCIT in the Community Setting}

Training. Fewer than half of the PCIT therapists who responded to the researcher were actively initiating PCIT cases during the course of the study. The current study provides further support to past research that suggests that many therapists who attend PCIT trainings do not actively provide PCIT following the training (Travis \& Brestan-Knight, 2012). Personal communication with some of the therapists indicated that common reasons for not delivering PCIT included small clinical caseloads due to administrative responsibilities and few referrals for young children with disruptive behavior.

The PCIT therapists who participated in the study had been trained in PCIT within the two years prior to the study. Some had completed few PCIT cases, and many reported that they had attempted PCIT with far more families than with whom they had completed PCIT. These therapists may have had much less experience coaching PDI than CDI. A therapist's experience with the PCIT model may impact his or her implementation of PCIT. However, the current study supports past research that suggests that community therapists achieve positive outcomes even with their first PCIT cases (e.g., Abrahamse et al., 2012; Pearl et al., 2011).

Implementation. Although therapists reported treatment integrity that was relatively high, it is the lowest treatment integrity reported in the PCIT literature (e.g., McCabe \& Yeh, 2009; Nixon et al., 2002; Schumann et al., 1998; Travis \& Brestan-Knight, 2012). This is not surprising given that most previous reports of treatment integrity are from efficacy studies with near perfect fidelity. The Travis and Brestan-Knight (2012) investigation is the only other published study to examine the treatment fidelity of community therapists using the videotapes 
submitted for review by a PCIT expert. The current study found lower treatment integrity with an overall treatment integrity at $74 \%$ for coaching sessions compared to $81 \%$ for coaching sessions in Travis and Brestan-Knights' (2012) study. Therapists in the other study may have submitted videotapes that were their "best" coaching sessions. Also, they may have found higher fidelity because the community therapists were recently trained in the model and receiving ongoing supervision in PCIT.

Treatment integrity in the current study reflects what community therapists were willing to report about what they actually do when not videotaped or closely monitored. Optimistically, the treatment integrity in the current study may indicate that community therapists were relatively honest in their report as past literature examined treatment integrity coded by independent observers watching videotapes (Travis \& Brestan-Knight, 2012). It is possible that true treatment integrity is lower than reported. Therapists' adherence to the model may drift as time elapses since their training and as they no longer receive ongoing consultation and supervision in the model. Also, integrity to the model may vary over time, not necessarily linearly, and with each family.

The aspects of treatment with the lowest integrity to the PCIT manual were reviewing changes on the ECBI and the DPICS as this occurred in about one quarter of sessions overall. In general, therapists reported that they liked the data-based and goal-oriented nature of PCIT. However, therapists' qualitative feedback indicated that giving the ECBI at every session was not believed to be necessary and was not always done. It is possible that therapists felt that giving the ECBI every few sessions, especially at the beginning of treatment, was sufficient to track changes in parent-reported child behavior at home. Alternatively, perhaps administering the ECBI was easy to skip if the therapist felt pressed for time because of the client arriving late or the client wanting to discuss child behavior or other events with the therapist. The financial burden of purchasing and administering a standardized measure at each session may also have been prohibitive. 
Aspects of PCIT with the highest integrity were coaching and coding. These may be essential components of PCIT to produce behavior change. This may explain why positive outcomes were demonstrated despite lower treatment integrity. However, the similarity between coaching by community therapists in the current sample and the coaching by graduate student therapists who receive close supervision and video monitoring remains unknown. Additionally, the reliability of the therapists' coding is unknown in this sample. Therapists may have believed that the parents met mastery, but their coding may have drifted from the DPICS definitions or may never have been reliable. Qualitative differences in coding and coaching may be closely linked to outcomes and drop out in PCIT. Also, the quality of training in PCIT may impact the accuracy of coding and quality of coaching.

Although integrity is low in comparison to other PCIT studies (e.g., McCabe \& Yeh, 2009; Nixon et al., 2002; Schumann et al., 1998; Travis \& Brestan-Knight, 2012), PCIT therapists reported relatively high overall fidelity, especially with regard to coding and coaching. Training of therapists in PCIT and their fidelity to the model may have impacted observed parent behavior. It is possible that higher fidelity may have resulted in larger effect sizes. The relative importance of components of the treatment integrity checklists remains unknown. For example, the importance of giving the ECBI at every session compared to coaching for the majority of the session is likely different for treatment outcomes. Overall, taken together with their responses on a qualitative survey, community therapists who continue to implement PCIT appear to appreciate the model and report relatively high fidelity.

Outcomes for families. The effect sizes of the changes reported in the current study are smaller than those reported in the PCIT literature including both efficacy and effectiveness research (e.g., Eisenstadt, et al., 1992; Galanter et al., 2012; McCabe \& Yeh, 2009; Nixon et al., 2002; Phillips et al., 2008; see Table 9). In the current study, intent-to-treat analyses were conducted and all participants were included regardless of their completion status. Thus, all participants were included regardless of whether they had completed PCIT, dropped out of PCIT, 
transferred to another form of therapy, or were still continuing with PCIT. This may contribute to the smaller effect sizes of the current study compared to other studies of PCIT.

Contrary to all the past literature in PCIT (e.g., Schumann et al., 1998; Nixon et al., 2002; McCabe \& Yeh, 2009; Pearl et al., 2011; Galanter et al., 2012), no changes were found in the PRIDE skills (e.g., Positive Parenting Composite) from the first to the second assessment. The inclusion criterion for PCIT families was to have received no more than two CDI coaching sessions. This may have contributed to the lack of change on observed CDI parent skills in the PCIT group. Even with this relatively lenient definition of the "beginning" of PCIT, the primary reason that PCIT families referred to the study were not enrolled was because they had received more than two coaching sessions by the time they could schedule a home visit with the researcher. However, it is possible that caregivers in the PCIT group had already made significant gains in "Do" and "Don't" Skills by the time the first assessment took place. A recency effect may have occurred as the PCIT families were continuing regular coaching in CDI skills in the clinic. At the second assessment, no families were in the CDI phase of treatment, and observations may indicate retention of skills. No families in either group were at "mastery" level of CDI (e.g., 10 labeled praise, 10 reflections, 10 behavior descriptions, no more than three combined commands, questions, and negative talk) at either assessment. However, it is possible that initial gains in CDI occur quickly, and it is these initial gains that maintain over time.

Unexpectedly, families in the PCIT group gave more indirect commands in relation to their total commands at the second assessment. These results contradict past literature that suggests an increase in the proportion of direct commands (e.g., Galanter et al., 2012). However, other studies have focused more on overall reduction in the frequency of commands (e.g., Nixon et al., 2002), and it is unknown how many of the commands were direct or indirect. The rationale for teaching caregivers to use direct commands is to achieve higher child compliance because direct commands are associated with higher child compliance (Forehand \& Scarboro, 1975; Stokes, Tempel, \& McNeil, 2011). In the current study, the opposite was found in that child 
compliance was higher at the second assessment and parents gave fewer direct commands. This finding may reflect caregiver drift from the PCIT procedure in response to the child's increased compliance rate (i.e., direct commands may be less necessary when children are complying with indirect commands). Alternatively, the lack of increase in direct commands could be due to the fact that most families had not completed PCIT at the time of the second assessment. Perhaps parents in the PCIT group used more indirect commands because they were not planning to follow-through with a warning statement or time-out for noncompliance. Alternatively, increased use of indirect commands may be related to improved child compliance. As child compliance improves, parents may be able to use more indirect commands. There are many possible explanations for the large effect in the opposite of the hypothesized direction for parent direct commands; however, the observation remains that child compliance significantly improved.

Generalization to the home. The current study provides support that improvements in child disruptive behavior generalize to the home. Contrary to expectations and past research (Naik-Polan \& Budd, 2008), changes in parent behavior were not observed in the home for the PRIDE skills. However, it is possible that changes in parent behavior had already occurred before the first assessment. At both assessments, the PRIDE skills of the parents in the PCIT group were significantly different from those of caregivers in the UC group. In the current study, child behavior improved at home although observed parent behavior did not match expectations.

The two families who had received BHRS supplemental to PCIT throughout the study reported difficulty with generalization to the home setting. They reported that the child did not engage in disruptive behavior in the clinic setting and continued to present with disruptive behavior in the home setting. This did not allow the parent to adequately practice skills in the clinic setting with the therapist present. These two families "gave up" on PCIT and opted to continue BHRS. However, these families did not experience improvement with the addition of BHRS, which is consistent with past research that has demonstrated that PCIT combined with wraparound services results in inferior outcomes compared to PCIT alone (Chaffin et al., 2004). 
Presumably, factors present in the clinic setting supported appropriate behavior while factors at home maintained disruptive behavior. Chaffin and colleagues (2004) speculated that wraparound services supplemental to PCIT "may have diluted interest in or attainment of behavioral parenting goals or that parents [who received supplemental services to PCIT] may have been inadvertently encouraged to attribute their parent-child problems to something other than their own parenting behavior" (Chaffin et al., 2004, p. 508). It is possible that continuing with BHRS gave the families hope of change without requiring the families to examine and identify the variables that were contributing to child disruptive behavior at home such as their own parenting behavior. Also, PCIT therapists reported that BHRS staff modeled behavior management strategies inconsistent with PCIT, which may have sent mixed signals to parents as to the most appropriate way to manage the child's behavior. Results suggest that PCIT generalizes to the home in many cases, although some families may struggle with achieving changes at home despite achieving changes in the clinic setting.

Dose and completion of PCIT. In addition to the differences in dose previously discussed, the relative success of PCIT compared to UC is also surprising because families received a relatively small number of PCIT sessions compared to past literature of PCIT. Past reports indicated that families typically received 12 to 18 sessions (e.g., Abrahamse et al., 2012; Lanier et al., 2011; McCabe\& Yeh, 2009; Nixon et al., 2002; Phillips et al., 2008). However, 12 to 18 sessions in the past research represents the number of sessions to complete PCIT. The median 9 sessions completed in the current study is similar to the number of sessions completed by families who dropped out of PCIT. Specifically, Abrahamse and colleagues (2012) reported that most families dropped out within the first 10 sessions. Lanier and colleagues (2011) reported that families who dropped out in CDI completed 5 sessions and families who dropped out in PDI completed 13 sessions. Previous literature has indicated that families who drop out of PCIT before completion make improvements but these are not as large as those made by families who complete treatment (e.g., Abrahamse et al., 2012; Lyon \& Budd, 2010), which may also partially 
explain why improvements in child behavior occurred even though only three families in the current study had completed PCIT.

Thirty-three percent of families dropped out of PCIT and thirty-three percent transferred to another treatment. Overall, $33 \%$ of families had completed or were continuing in PCIT at the time of the second assessment. Drop out from PCIT in the current study was similar to drop out from UC. However, anecdotally more families transferred from PCIT to another treatment than in UC. The main reason for switching to BHRS was that the child received a diagnosis of ASD. Contrary to the previously reported evaluations of PCIT effectiveness in which most drop out occurred during CDI (e.g., Abrahamse et al., 2012; Lanier et al., 2011; Pearl et al., 2011; Phillips et al., 2008), a relatively equal number of participants in the current study dropped out of PCIT or transferred to another treatment modality during CDI and PDI. Anecdotally, families appeared more likely to drop out of treatment during CDI and transfer to another treatment (e.g., BHRS) in PDI. Additionally, drop out or transfer from PCIT was not significantly different than the drop out and transfer in the UC group. This suggests that the dropout rate for PCIT may be representative of families who seek therapy in general and may not be due to characteristics of PCIT in particular.

\section{Usual Care: Behavioral Health Rehabilitative Services}

Effect sizes for BHRS ranged from .22 to .39 for child behavior measures indicating small effect sizes. These results support past literature of BHRS that "suggest a general trend in effectiveness" (Knapp \& Reed, 2010, pg. 5). However, these effect sizes are smaller than research that suggested a medium effect size in parent-reported treatment outcomes (Toffalo, 2000). It is possible that inclusion of families who dropped out of BHRS decreased the observed effect sizes in the current study. BHRS has been established in Pennsylvania for about 30 years (Knapp \& Reed, 2010). Based on personal communications, a common belief held by the community therapists in BHRS was that young children were most effectively treated in the natural environment where their behaviors occurred. The popularity of BHRS may reflect 
concerns that typical outpatient therapy is insufficient to treat young children with aggressive and disruptive behavior. Also, BHRS' popularity may reflect its familiarity to clinicians and families who live in the region.

The description of BHRS is consistent with past literature that has found that evidencebased practices are implemented with low intensity in usual care settings (Garland et al., 2010). Based on reported descriptions of treatment, BHRS staff recommended common behavior management strategies and frequently modeled implementation of behavior management procedures while in the home. However, BHRS is not best characterized as behavioral parent training. Treatment descriptions and the interactions between the researcher, families, and therapists did not indicate any evidence of planned skills transfer from the therapeutic team to the caregiver or to times when the therapeutic team was not present. Modeling of therapeutic strategies by therapeutic staff was the presumed mechanism of change. There is little evidence of other methods of increasing parental use of behavior management strategies. For example, homework was provided in only $15.8 \%$ of "typical" weeks and reviewed during $4.8 \%$ of "typical" weeks. Although Behavior Specialist Consultants reported that they spent time with the caregiver and child together and commented on interactions during most "typical" weeks, the quality and frequency of their possible "coaching" is unknown. Kaminski and colleagues (2008) reported that modeling without parental in-session practice of behavior management skills with the child had smaller effects than when in-session practice with the parent and child was included. The focus on modeling rather than coaching may explain the small effect sizes found in the UC condition despite the approximate 113.5 median hours of in-home billable service per family during the course of the current study.

Although PCIT had larger effect sizes than BHRS, BHRS may have produced valuable outcomes in child functioning that were not measured in the current study. BHRS staff can provide services in the school setting, and outcomes in the school setting were not measured in the current study. BHRS is well suited to providing the necessary hours for discrete-trial training 
for language acquisition with children with ASD. Additionally, BHRS staff are able to prompt and reinforce appropriate social interactions in community settings, such as at birthday parties, scouts, or athletic events. BHRS may produce valuable outcomes that were not measured in the current study. However, incorporating behavioral parent training and increasing the use of existing therapeutic components may produce better outcomes in home-based wraparound interventions.

\section{Further Implications of the Current Study}

The current study suggests that young children with disruptive behavior can be treated effectively in an outpatient setting. In-home services do not necessarily promote generalization to times when therapeutic staff members are not present. Also, more hours of therapeutic services do not necessarily produce better outcomes. It should be noted that the hours of service to produce sufficient treatment gains is likely highly related to factors in addition to therapy modality, such as parental motivation and engagement in therapy and the severity of child problem behavior. The intensity and type of therapeutic interventions utilized may be more critical in decreasing child disruptive behavior than the number of hours of therapeutic services. The results imply that training of parents in behavior management strategies is of utmost importance in changing child disruptive behavior.

With regard to dissemination of evidence-based treatments, the current study suggests that many therapists trained in an evidence-based treatment may not implement it at all following training. Also, therapists may not implement the evidence-based treatment often, and this may impact the quality of its implementation when therapists do use the evidence-based treatment. Yet, even though PCIT was implemented with lower treatment integrity in this investigation than in previous efficacy studies, PCIT was found to produce good treatment outcomes. Also, the PCIT therapists in this study were relatively inexperienced, suggesting that newly trained therapists can achieve good outcomes even with their first few cases. Additionally, the current findings suggest that treatment effects of PCIT may be present within the first few weeks of 
treatment. Similar drop-out rates between PCIT and UC in this investigation suggest that treatment drop out found in the literature of evidence-based practices may reflect difficulty engaging families in therapy in general rather than a concern specific to evidence-based treatments.

PCIT, and behavioral parent training in general, may be the optimal treatment when a young child presents primarily with disruptive behavior such as tantrums, noncompliance, and aggression. BHRS may continue to have value when presenting concerns are functional language deficits, deficits in social interactions with peers, or behavior problems in the school setting. If disruptive behavior, social skills deficits, and behavior concerns in school are all presenting concerns, then completion of PCIT may be indicated before initiation of BHRS services. BHRS supplemental to PCIT may be contraindicated, although results may be different if BHRS staff implemented behavior interventions consistent with PCIT and actively promoted generalization through parental practice of behavior management strategies with the child.

\section{Limitations of the Current Study}

Small sample size and type II error. Failure to follow-up with $17 \%$ of the original sample diminished the statistical power to find significant interactions between the groups and increased the likelihood of failing to find a true difference when it existed. Thus, despite medium to large effect sizes between groups on dependent measures at the second assessment, almost all differences failed to reach statistical significance at the alpha level of .05. The small sample size also contributed to violations of the assumptions of statistical tests, such as sphericity. If a true difference exists, the larger effect sizes observed in the PCIT group suggest that PCIT may be superior to UC for disruptive behavior in young children.

Lack of randomization to condition. Conclusions from this study are limited given the lack of randomization to condition. Significant differences in demographic differences were found between groups regarding child sex, parent age, and parental history of counseling. Specifically, significantly more girls were in the PCIT group, caregivers in the PCIT group were 
significantly older, more caregivers in the PCIT group had received counseling than in the UC group, and more children in the UC were prescribed medications. These differences may reflect a selection bias of the professionals in the community who made decisions as to who met medical necessity for BHRS and who would be a "good" case for PCIT. Also, it is possible that the therapists who completed PCIT training, continued to implement it, and referred participants tot the study may have different characteristics than typical therapists in the community setting. For example, the PCIT therapists in the current study may have been more motivated or enthusiastic. Randomization to condition would decrease the plausibility that selection bias is responsible for differences in treatment effects.

Lack of true "pretreatment." Conclusions in the current study are limited by lack of a true "pretreatment" assessment. Inclusion of PCIT families who had completed up to two CDI coaching sessions prevented examination of the treatment gains from the true "beginning" of PCIT. Additionally, families in the UC group had received as much as two months of services prior to the first assessment. Treatment hours received prior to the first assessment were not included in the reported treatment dose for either group. The reported number of PCIT sessions should be considered an underestimate of total PCIT sessions. Changes in parent and child behavior may have been occurring before the first assessment occurred. This may have decreased observed effect sizes on all measures. If the first assessment took place before the CDI Teach session, as occurred in other PCIT studies, larger effects may have been found. Also, effect sizes for UC may have been larger if the first assessment had taken place before any services had been delivered. Furthermore, the conclusions of this study are limited because the amount of therapy services before the first assessment varied. Even with the lenient criteria to define the "beginning" of treatment, reaching the third CDI coaching session and length of time in therapy were the primary barriers in recruiting and enrolling participants in the study.

Reactivity and socially desirable responding. Another limitation is that reactivity to videotaping may have altered parent-child interactions. Parents sometimes reported that the child 
either acted worse or better than typical during the observation. The researcher made efforts to minimize reactivity. For example, families engaged in a 5-minute "warm up" period prior to videotaping during which the researcher sat with the camera facing the parent and child. Also, the researcher positioned herself and the camera unobtrusively and refrained from moving during the observation as much as possible. However, the presence of an unknown person with a video camera may have altered both parent and child behavior.

It is possible that caregivers or therapists responded on questionnaires in a socially desirable way, therefore limiting the conclusions of this study. The community therapists' report of treatment fidelity remains subjective because it lacks independent reliability. To minimize socially desirable responding for families, the researcher emphasized that she would not share identifiable results with the therapist or agency. To minimize socially desirable responding on treatment integrity questionnaires for therapists, the researcher told the therapists that she wanted to know what the therapists "really did," because she already knew what the manual said.

Videotaping was not typical for any of the therapists or families who participated in the study. Videotaping equipment was not typically available in these community settings for professional purposes. Attempting to introduce videotaping into these settings during clinical practice to examine treatment integrity may have altered the nature of the services because of reactivity or novelty. Family reactivity to videotaped observations and parental and therapist reactivity to questionnaires may have altered the results of the study.

Representativeness of PCIT. The PCIT therapists in the current study volunteered to refer their PCIT families and fill out questionnaires about treatment. The PCIT therapists who participated may have been more engaged in or feel more comfortable with the model than therapists who did not refer participants. Also, the PCIT therapists varied in their experience with the model, and some had not completed any PCIT cases. Results of PCIT may be different with therapists who have more experience with PCIT. PCIT therapists can be trained using a variety 
of formats, and not all PCIT therapists may be trained in the manner of those in the current study. The PCIT therapists and the PCIT delivered in this study may not be representative of all PCIT.

Representativeness of UC. UC in this study may not represent UC for young children with disruptive behavior in general. The UC care in the current study represents the typical treatment for young children with disruptive behavior in the geographic region where the study took place. Although BHRS represents the true "usual care" for the population in this study, BHRS may not reflect usual care for young children with disruptive behavior in other geographic regions. UC in other geographic regions may take place in the clinic and may not provide so many hours of service. It should be noted that it is difficult to describe usual care, and each study evaluating usual care must make strong efforts to describe it. Usual care may be different in each study.

\section{Future Directions}

More research is needed examining the dissemination, therapist training, implementation, and outcomes of evidence-based practices and treatments in community settings. Effectiveness studies raise questions that are best examined in the controlled environment of an efficacy study. Efficacy studies develop practices and treatments that must be tested in the less controlled "real world." The current study raises many questions for further research.

First, a larger and better-funded examination of community PCIT and UC with more therapists and families is warranted. In general, more examination of PCIT compared to other treatments is needed. Randomization of families may be difficult given real-world constraints; however, it may be possible to randomize agencies for training and implementation of PCIT. The therapeutic practices should be examined more intensively using audio or visual recording of sessions. Better data regarding qualitative aspects of PCIT in the community setting would be valuable. Assessment should take place before treatment begins and should occur frequently throughout treatment to increase understanding of when change takes place. Lastly, the costeffectiveness of PCIT compared to other treatments should be examined. 
Second, future research should examine factors that influence the effectiveness of PCIT. Component analysis should be conducted to explore which treatment components are necessary and sufficient to produce treatment gains. For example, the amount of time spent coaching and the content of coaching may impact treatment outcomes and drop out. Administering the ECBI every session may not be necessary. An understanding of factors associated with coaching that produce the best outcomes should be explored. The current study raised possible biases about which cases might be "good" for PCIT, and research should explore whether certain variables (i.e., child sex, parent age, parental psychopathology) impact outcomes or the number of sessions needed to produce desired outcomes. Generalization from the clinic to the home setting is an area in need of further research, especially when treatment gains are not generalizing to the home setting.

Third, more research is needed to understand how to best train community professionals in PCIT. For example, research should examine factors that contribute to therapists continuing to use PCIT after training. This may lead to identification of the best candidates to receive training in PCIT and minimize wasted money on training therapists who will not implement the treatment. Institutional barriers to implementation of PCIT should be examined in order to identify methods to overcome these barriers. The effectiveness of PCIT in the community rests on the effective training of community professionals. The accuracy of therapist coding and quality of therapist coaching likely influence changes in disruptive child behavior. Methods of training that promote future continued implementation and fidelity to the treatment should be examined.

Fourth, it may be valuable to explore the outcomes of PCIT when perceived strengths of other treatments are incorporated. It would be interesting to examine a high-dose, short-term implementation of "Intensive" PCIT in the home setting in terms of child behavior outcomes and retention in treatment. For example, supplemental PCIT sessions in the home setting in addition to clinic PCIT may promote generalization in difficult cases. A higher dose of PCIT may stabilize families with a high frequency of aggression and property destruction. Also, in-home 
PCIT may decrease barriers to treatment for families with transportation difficulties.

Additionally, the outcomes of PCIT with supplemental services should be explored. The addition of supplemental services may not dilute the treatment outcomes if the services are targeting different treatment goals. For example, some perceived weaknesses of PCIT may be removed with the addition of Therapeutic Support Staff to facilitate social interactions with peers or address deficits in functional communication. Also, therapeutic Support Staff may be helpful to take care of siblings and minimize distractions during in-home PCIT sessions. The variables that hinder PCIT when combined with wraparound services should be examined to determine if there would be a way to implement supplemental services without decreasing the effectiveness of PCIT. Also, wraparound services, such as BHRS, may be more effective when behavioral parent training with an emphasis on coding and coaching parent behavior is incorporated. Also, increased utilization of standardized assessment measures in clinical practice on a weekly basis would help evaluate the effectiveness of treatment with each individual family. An "ideal" treatment may include weekly assessment of parent and child behavior via direct observation, weekly feedback to caregivers, supplemental in-home coaching sessions with the help of Therapeutic Support Staff as indicated, increased funding for reliable transportation to the clinic, a focus on coaching parents with their children during the sessions, and goals that include increased positive parent-child interactions as well as time-out. Much more research is needed to examine effective models for implementing behavioral parent training in the community setting.

Lastly, more research is needed to examine how to increase parental engagement and motivation for change. Drop out from treatment prevents children and families from reaping the full benefits of an evidence-based treatment. Understanding predictors of drop out from treatment as well as strategies to increase engagement in treatment may increase the effectiveness of evidence-based treatments.

\section{Summary}


The current investigation suggests that PCIT may be a cost-effective treatment for young children with disruptive behavior disorders because it may produce larger effects with fewer service hours. The current study contributes to the PCIT literature at large by using a standardized, bi-modal assessment and including reliability of observational data to examine the outcomes of PCIT delivered by community therapists. Additionally, this investigation describes and examines the outcomes of usual care, which was predominantly Behavioral Health and Rehabilitative Services ("Wraparound"). Thus, the current study contributes to the knowledge of usual care and wraparound services in community settings as well as the effectiveness of PCIT in comparison to usual care.

The current study implies that PCIT delivered by community therapists may produce commensurate or larger effects with fewer service hours for children with disruptive behavior than usual care. This investigation supports past research that indicates that few therapists trained in PCIT continue to implement it regularly (Travis \& Brestan-Knight, 2012). Those therapists who continue to implement PCIT reported relatively high treatment fidelity, especially with regard to coding and coaching parent-child interactions. They also reported to find PCIT to be an acceptable method of treatment for young children with disruptive behavior.

This research supports past research suggesting that wraparound services supplemental to PCIT may be contraindicated (e.g., Chaffin et al., 2004); however, wraparound services may impact outcomes that were not examined in the current study. Incorporation of behavioral parent training with an emphasis on coaching parents with their children may increase the effects of wraparound services. Alternatively, "Intensive" PCIT may provide even greater results than either of the studied interventions. In this model, a co-therapy team of a master's level PCIT therapist and bachelor's-level support staff member (helping with sibling care) could provide a high dose of PCIT in both the home and clinic. This intensive PCIT model could provide an effective treatment for multi-problem families with high frequency aggression who may be unlikely to regularly attend outpatient appointments and may require a higher level of care than is 
provided in a weekly outpatient modality. For families struggling to generalize treatment gains to the home, in-home coaching sessions may provide an opportunity for therapists to examine barriers to generalization and to coach parents as misbehavior occurs.

Although the results of this study suggest that PCIT produces favorable outcomes in community mental health, the small sample size and lack of randomization to condition limit the conclusions from this study. Further research comparing PCIT to UC with more families is warranted. Additionally, randomizing agencies to either PCIT or an alternative intervention would better test the effects of PCIT compared to UC in the community. 
References

Abidin, R. R. (1995). Parenting Stress Index: Professional Manual (3rd ed.). Odessa, FL:

Pscyhological Assessment Resources.

Abrahamse, M. E., Junger, M., Chavannes, E. L., Coelman, J. G., Boer, F., \& Lindauer, J. L. (2012). Parent-child interaction therapy for preschool children with disruptive behavior problems in the Netherlands. Child and Adolescent Psychiatry and Mental Health, 6, $1-$ 9.

Achenbach, T. M., \& Rescorla, L. A. (2000). Manual for ASEBA preschool forms \& profiles. Burlington, VT: University of Vermont, Research Center for Children, Youth, \& Families.

Achenbach, T. M., \& Rescorla, L. A. (2001). Manual for ASEBA school-age forms \& profiles. Burlington, VT: University of Vermont, Research Center for Children, Youth, \& Families.

Acton, R. G., \& During, S. M. (1992). Preliminary results of aggression management training for aggressive parents. Journal of Interpersonal Violence, 7, 410-417.

American Psychiatric Association (2000). Diagnostic and Statistical Manual of Mental Disorders (4 $4^{\text {th }}$ Edition) Text Revision. Arlington: American Psychiatric Association.

Bagner, D. M., \& Eyberg, S. M. (2007). Parent-child interaction therapy for disruptive behavior in children with mental retardation: A randomized controlled trial. Journal of Clinical Child and Adolescent Psychology, 36, 418-429.

Barkley, R. S. (1997). Defiant Children (2 ${ }^{\text {nd }}$ Ed. ) New York: The Guilford.

Berkovits, M. D., O’Brien, K. A., Carter, C. G., \& Eyberg, S. M. (2010). Early identification and intervention for behavior problems in primary care: A comparison of two abbreviated versions of parent-child interaction therapy. Behavior Therapy, 41, 375387.

Borrego, J., Gutow, M. R., Reicher, S., \& Barker, C. H. (2008). Parent-child interaction 
therapy with domestic violence populations. Journal of Family Violence, 23, 495-505. Borrego, J., Urquiza, A. J., Rasmussen, R. A., \& Zebell, N. (1999). Parent-child interaction therapy with a family at high risk for physical abuse. Child Maltreatment, 4, 331-342.

Brestan, E. V., Eyberg, S. M., Boggs, S. R., \& Algina, J. (1997). Parent-child interaction therapy: Parents' perceptions of untreated siblings. Child and Family Therapy, 19, 13 28.

Brestan, E., Jacobs., J., Rayfield, A., \& Eyberg, S. M. (1999). A consumer satisfaction measure for parent-child treatments and its relationship to measures of child behavior change. Behavior Therapy, 30, 17-30.

Bruns, E., Burchard, J., \& Yoe, J. (1995). Evaluating the Vermont system of care: Outcomes associated with community-based wraparound services. Journal of Child and Family Studies, 4, 321-339.

Capage, L. C., Bennett, G. M., \& McNeil, C. B. (2001). A comparison between African American and Caucasian children referred for treatment of disruptive behavior disorders. Child and Family Behavior Therapy, 23, $1-14$.

Chaffin, M., Silvosky, J. F., Funderburk, B., Valle, L. A., Brestan, E. V., Balachova, R., Jackson, S., Lensgraf, J., \& Bonner, B. (2004). Parent-child interaction therapy with physically abusive parents: Efficacy for reducing future abuse reports. Journal of Consulting and Clinical Psychology, 72, 500-510.

Chaffin, M. Valle, L., Funderburk, B., Gurwitch, R., Silovsky, J., Bard, D., McCoy, C., \& Kees, M. (2009). A motivational intervention can improve retention in PCIT for low-motivation child welfare clients. Child Maltreatment, 14, 356-368.

Chambless, D. L., Baker, M. J., Baucom, D. H., Beutler, L. E., Calhoun, K. S., Crits-Christoph, P., ... Woody, S. R. (1998). Update on empirically validated therapies, II. The Clinical Psychologist, 51, $3-16$.

Choate, M. L., Pincus, D. B., Eyberg, S. M., \& Barlow, D. H. (2005). Parent-child interaction 
therapy for treatment of separation anxiety disorder in young children: A pilot study. Cognitive and Behavioral Practice, 12, 126-135.

Eisenstadt, T. H., Eyberg, S., M., McNeil, C. B., Newcomb, K., \& Funderburk, B. (1993). Parent-child interaction therapy with behavior problem children: Relative effectiveness of two stages and overall treatment outcome. Journal of Clinical Child Psychology, 12, 4251.

Eyberg, S. M. (1988). Parent-child interaction therapy: Integration of traditional and behavioral concerns. Child and Family Behavior Therapy, 10, 33-46.

Eyberg, S. M., \& Funderburk, B (2011). Parent-Child Interaction Therapy Protocol. Gainesville, FL: PCIT International.

Eyberg, S. M., Funderburk, B. W., Hembree-Kigin, T. L., McNeil, C. B., Querido, J. G., \& Hood, K. (2001). Parent-child interaction therapy with behavior problem children: One and two year maintenance of treatment effects in the family. Child and Family Behavior Therapy, $23,1-20$.

Eyberg, S. M., \& Matarazzo, R. G. (1980). Training parents as therapists: A comparison between individual parent-child interaction training and parent group didactic training. Journal of Clinical Psychology, 36, 492-499.

Eyberg, S. M., Nelson, M. M., Duke, M., \& Boggs, S. R. (2005). Manual for the Dyadic Parent-Child Interaction Coding System (3rd ed.). University of Florida: Child Study Laboratory.

Eyberg, S. M., Nelson, M. M., \& Boggs, S. R. (2008). Evidence-based psychosocial treatments for children and adolescents with disruptive behavior. Journal of Clinical Child and Adolescent Psychology, 37, 215-237.

Eyberg, S., \& Pincus, D. (1999). Eyberg child behavior inventory \& Sutter-Eyberg student behavior inventory-revised. Odessa, FL: Psychological Assessment Resources.

Eyberg, S. M., \& Robsinson, E. A. (1982). Parent-child interaction training: Effects on 
family functioning. Journal of Clinical Child Psychology, 11, 130-137.

Faul, F., Erdfelder, E., Lang., A-G., \& Buchner, A. (2007). G*Power 3: A flexible statistical power analysis program for the social, behavioral, and biomedical sciences. Behavior Research Methods, 39, 175-191.

Fernandez, M.A., Chase, R.M., Ingalls, C.A., \& Eyberg, S.M. (2006). The Abridged Workbook: Coder Training Manual for the Dyadic Parent-Child Interaction Coding System ( $3^{\text {rd }}$ ed.). Retrieved October 20, 2009 from www.pcit.org.

Forehand, R., \& Scarboro, M. E. (1975). An analysis of children's oppositional behavior. Journal of Abnormal Child Psychology, 3, 27-31.

Frazier, S. L., Formoso, D., Birman, D., \& Atkins, M. S. (2008). Closing the research to practice gap: Redefining feasibility. Clinical Psychology: Science and Practice, 15, 125129.

Galanter, R., Self-Brown, S., Valente, J. R., Dorsey, S., Whitaker, D. J., Bertuglia-Haley, M., Priesto, M. (2012). Effectiveness of parent-child interaction therapy delivered to at-risk families in the home setting. Child \& Family Behavior Therapy, 34, 177-196. DOI: $10.1080 / 07317107.2012 .707079$

Garland, A. F., Brookman-Frazee, L. B., Hurlburt, M S., Accurso, E. C., Zoffness, R. J., HaineSchlagel, R., \& Ganger, W. (2010). Mental health care for children with disruptive behavior problems: A view inside therapists' offices. Psychiatric Services, 61, 788- 795.

Garland, A. F., Hurlburt, M. S., Brookman-Frazee, L., Taylor, R. M., \& Accurso, E. C. (2010). Methodological challenges of characterizing usual care psychotherapeutic practice. Administration and Policy in Mental Health, 37, 208-220.

Herschell, A D., Calzada, E. J., Eyberg, S. M., \& McNeil, C. B. (2002a). Clinical issues in parentchild interaction therapy. Cognitive and Behavioral Practice, 9, 16-27.

Herschell, A. D., Calzada, E. J., Eyberg, S. M., \& McNeil, C. B. (2002b). Parent-child interaction therapy: New directions in research. Cognitive and Behavioral Practice, 9, 9-16. 
Herschell, A. D., McNeil, C. B., Urquiza, A. J., McGrath, J. M., Zebell, N. M., Timmer, S. G., \& Porter, A. (2009). Evaluation of a treatment manual and workshops for disseminating, parent-child interaction therapy. Adminstration and Policy in Mental Health, 36, 63-81.

Hoagwood, K., Hibbs, E., Brent, D., \& Jensen, P. (1995). Introduction to the special section: Efficacy and effectiveness in studies of child and adolescent psychotherapy. Journal of Consulting and Clinical Psychology, 63, 683-687.

Hoagwood., K., \& Kolko, D. J. (2009). Introduction to the special section on practice contexts: A glimpse into the nether world of public mental health services for children and families. Administration and Policy in Mental Health, 36, 35-36.

Hohmann, A. A., \& Shear, M. K. (2002). Community-based intervention research: Coping with the "noise" of real life in study design. American Journal of Psychiatry, 159, 201-207.

Hood, K., \& Eyberg, S. M. (2003). Outcomes of parent-child interaction therapy: Mothers’ reports on maintenance three to six years after treatment. The Family Journal: Counseling and Therapy for Couples and Families, 8, 180-186.

Kaminski, J. W., Valle, L. A., Filene, J. H., \& Boyle, C. L. (2008). A meta-analytic review of components associated with parent training program effectiveness. Journal of Abnormal Child Psychology, 36, 567-589.

Knapp, S., \& Reed, R. (2010). Does (or why does) BHRS work? The Pennsylvania Psychologists Update, 5-6.

Lanier, P., Kohl, P. L., Benz, J., Swinger, D., \& Drake, B. (2012). Preventing maltreatrement with a community-based implementation of parent-child interaction therapy. Journal of Child and Family Studies, 23, 449-460. DOI: 10.1007/s10826-012-9708-8

Lanier, P., Kohl, P. L., Benz, J., Swinger, D., Mousette, P., \& Drake, B. (2011). Parent-child 
interaction therapy in a community setting: Examining outcomes, attrition, and treatment setting. Research on Social Work Practice, 21, 689-698. DOI:

$10.117 / 1049731511406551$

Lenze, S. N., Pautsch, J., \& Luby, J., (2011). Parent-child interaction therapy emotion development: A novel treatment for depression in preschool children. Depression and Anxiety, 28, 153-159.

Lyon, A. R., \& Budd, K. S. (2010). A community mental health implementation nof parent-child interaction therapy (PCIT). Journal of Child and Family Studies, 19, 654-668.

Masse, J. J. (2010). Examining the efficacy of parent-child interaction therapy with highfunctioning autism. Dissertation Abstracts International: Section B: The Sciences and Engineering, 70, 6658.

Matos, M., Bauermeister, J. J., \& Bernal, G. (2009). Parent-child interaction therapy for Puerto Rican preschool children with ADHD and behavior problems: A pilot efficacy study. Family Process, 48, $232-252$.

McCabe, K., \& Yeh, M. (2009). Parent-child interaction therapy for Mexican Americans: A randomized clinical trial. Journal of Clinical Child \& Adolescent Psychology, 38, 753-759.

McCart, M. R., Priester, P. E., Hobart, D. W., \& Azen, R. (2006). Differential effectiveness of behavioral parent-training and cognitive-behavioral therapy for antisocial youth: A metaanalysis. Journal of Abnormal Child Psychology, 24, 527-543.

McMahon, R. J., \& Forehand, R. L. (2003). Helping the noncompliant child: Family-based treatment for oppositional behavior ( $2^{\text {nd }}$ Ed.) New York: The Guilford.

McNeil, C. B., Eyberg, S., Eisenstadt, T. H., Newcomb, K., \& Funderburk, B. (1991). Parent-child interaction therapy with behavior problem children: Generalization of treatment effects to the school setting. Journal of Clinical Child Psychology, 20, 140151. 
McNeil, C. B., \& Hembree-Kigin, T. (2011). Parent-Child Interaction Therapy $\left(2^{\text {nd }}\right.$ Ed.). New York, NY: Springer.

McNeil, C. B., Herschell, A. D., Gurwitch, R. H., \& Clemens-Mowrer, L. (2005). Training foster parents in parent-child interaction therapy. Education and Treatment of Children, 28, 182-196.

Naik-Polan, A. T., \& Budd, K. S. (2008). Stimulus generalization of parenting skills during parent-child interaction therapy. Journal of Early and Intensive Behavior Intervention, 5, $71-92$.

Nixon, R. D. V., Sweeney, L., Erickson, D. B., \& Touyz, S. W. (2003). Parent-child interaction therapy: A comparison of standard and abbreviated treatments for oppositional defiant preschoolers. Journal of Consulting and Clinical Psychology, 71, $251-260$.

Pade, H., Taube, D. O., Aalborg, A E., \& Reiser, P. J. (2006). An immediate and longterm study of a temperament and parent-child interaction therapy based community program for preschoolers with behavior problems. Child \& Family Behavior Therapy, 28, $1-28$.

PCIT International (2013). Training Requirements for Certification as a Level I Trainer ("Within Program Trainer”). Retreived from www.pcit.org on February 2, 2014.

PCIT International (2013). Training Requirements for Certification as a Level II Trainer. Retreived from www.pcit.org on February 2, 2014.

PCIT International (2013). Training Requirements for Certification as a Master Trainer. Retreived from www.pcit.org on February 2, 2014.

PCIT International (2013). Training Requirements for Certification as a PCIT Therapist. Retreived from www.pcit.org on February 2, 2014.

Pearl, E., Thieken, L., Olafson, E., Boat, B., Connelly, L., Barnes, J., \& Putnam, F. 
(2011). Effectiveness of community dissemination of Parent-Child Interaction Therapy. Psychological Trauma: Theory, Research, Practice, and Policy, 1-10. DOI: 10.1037/a0022948.

Pennsylvania Department of Public Welfare (2001). Guidelines for Best Practice in Child and Adolescent Mental Health Care. Retrieved from www.dpw.state.pa.us/cs/groups/public/documents/manual/s_001583.pdf on February 2, 2014.

Phillips, J., Morgan, S., Cawthorne, K., \& Barnett, B. (2008). Pilot evaluation of parent-child interaction therapy delivered in an Australian community early childhood clinical setting. The Royal Australian and New Zealand College of Psychiatry, 42, 712- 719.

Prinz, R. J., Sanders, M. R., Shapiro, C. J., Whitaker, D. J., \& Lutzker, J. R. (2009). Populationbased prevention of child maltreatment: The U.S. triple P system population trial. Prevention Science, 10, 1-12.

Reitman, D., \& McMahon, R. J. (2013). Constance “Connie” Hanf (1927-2002): The mentor and the model. Cognitive and Behavioral Practice, 20, 106-116.

Sanders, M. R., Calam, R., Durand, M., Liversidge, \& Carmont, S. A. (2008). Does self-directed and web-based support for parents enhance the effects of viewing a reality television series based on the triple P- positive parenting programme? Journal of Child Psychology and Psychiatry, 49, 924-932.

Sanders, M. R., \& Kirby, J. N. (2012). Consumer engagement and the development, evaluation, and dissemination of evidence-based parenting programs. Behavior Therapy, 43, 236250.

Sanders, M. R., Markie-Dadds, C., Tully, L. A., \& Bor, W. (2000). The triple P positive parenting program: A comparison of enhanced, standard, and self-directed behavioral family intervention for parents of children with early onset conduct problems. Journal of Consulting and Clinical Psychology, 68, 624-640. 
Sanders, M. R., Montgomery, D., \& Brechman-Toussaint, M. (2000). The mass media and the prevention of child behavior problems: The evaluation of a television series to promote positive outcomes for parents and their children. Journal of Child Psychology and Psychiatry, 41, 939-948.

Sanders, M. R., Ralph, A., Sofronoff, K., Gardiner, P., Thompson, R., Dwyer, S., \& Bidwell, K. (2008). Every family: A population approach to reducing behavioral and emotional problems in children making the transition to school. Journal of Primary Prevention, 29, 197-222.

Schuhmann, E. M., Foote, R. C., Eyberg, S. M., Boggs, S. R., \& Algina, J. (1998). Efficacy of parent-child interaction therapy: Interim report of a randomized trial with short-term maintenance. Journal of Clinical Child Psychology, 27, 34-45.

Showers, B., \& Joyce, B. (1996). The evolution of peer coaching. Educational Leadership, 53, $12-16$.

Solomon, M., Ono, M., Timmer, S., \& Goodlin-Jones, B. (2008). The effectiveness of parent-child interaction therapy for families of children on the autism spectrum. Journal of Autism and Developmental Disorders, 38, 1767-1776.

Stokes, J. O., Tempel, A. B., \& McNeil, C. B. (2011, November). Antecedents and consequences of compliance in high risk families. Poster presentation at the annual meeting of the Association for Behavioral and Cognitive Therapies, Toronto, Canada.

Timmer, S. G., Urquiza, A. J., \& Zebell, N. M. (2006). Challenging foster caregiver- maltreated child relationships: The effectiveness of parent-child interaction therapy. Children and Youth Services Review, 28, 1- 19.

Timmer, S. G., Urquiza, A. J., Zebell, N. M., \& McGrath, J. M. (2005). Parent-child interaction therapy: Application to maltreating parent-child dyads. Child Abuse and Neglect, 29, $825-842$.

Timmer, S. G., Zebell, N. M., Culver, M. A., \& Urquiza, A. J. (2010). Efficacy of adjunct 
in-home coaching to improve outcomes in parent-child interaction therapy. Research on Social Work Practice, 20, 35-45.

Thomas, R., \& Zimmer-Gembeck, M. J. (2007). Behavioral outcomes of parent-child interaction therapy and triple $\mathrm{p}$ - positive parenting program: A review and meta-analysis. Journal of Abnormal Child Psychology, 35, 475 - 495. DOI: 10.1007/s10802-007-9104-9.

Toffalo, D. A. (2000). An investigation of treatment integrity and outcomes in wraparound services. Journal of Child and Family Studies, 9, 351 -361.

Travis, J. K., \& Brestan-Knight, E. (2013). A pilot study examining trainee treatment session fidelity when parent-child interaction therapy (PCIT) is implemented in community settings. The Journal of Behavioral Health Services and Research, 40, 342 - 354.

VanDerBerg, J. E., (1993). Integration of individualized mental health services into the system of care for children and adolescents. Administration and Policy in Mental Health, 20, $247-$ 257.

Ware, L., McNeil, C. B., Masse, J., \& Stevens, S. (2008). Efficacy of in-home parent-child interaction therapy. Child and Family Behavior Therapy, 30, 99-126.

Webster-Stratton, C., \& Eyberg, S. M. (1982). Child temperament: Relationship with child behavior problems and parent-child interactions. Journal of Clinical Child Psychology, $11,123-129$.

Webster-Stratton, C. (1984). Randomized trial of two parent training programs for families with conduct disorder children. Journal of Consulting and Clinical Psychology, 52, 666-678.

Webster-Stratton, C. (1992). Incredible years: A trouble-shooting guide for parents of children age 3 to 8 . Umbrella Press: Toronto.

Webster-Stratton, C., \& Hammond, M. (1990). Predictors of treatment outcome in parent training for families with conduct problem children. Behavior Therapy, 21, 319-337.

Webster-Stratton, C., \& Hammond, M. (1997). Treating children with early-onset conduct 
problems: A comparison of child and parent training interventions. Journal of Consulting and Clinical Psychology, 65, 93-109.

Weisz, J. R., Donenberg, G. R., Han, S. S., \& Weiss, B. (1995). Bridging the gap between laboratory and clinic in child and adolescent psychotherapy. Journal of Consulting and Clinical Psychology, 63, 688-701.

Weisz, J. R., \& Jensen, P. S. (1999). Efficacy and effectiveness of child and adolescent psychotherapy and pharmacotherapy. Mental Health Services Research, 1, 125-157.

Weisz, J. R., Jensen-Doss, A., \& Hawley, K. M. (2005). Youth psychotherapy outcome research: A review and critique of the evidence base. Annual Review of Psychology, 56, 337-363.

Weisz, J. R., Jensen-Doss, A., \& Hawley, K. M. (2006). Evidence-based youth psychotherapies versus usual clinical care. American Psychologist, 61, 671-689.

Weisz, J. R., Weiss, B., \& Donenberg, G. R. (1992). The lab versus the clinic: Effects of child and adolescent psychotherapy. American Psychologist, 47, 1578- 1585. 
Table 1.

Definitions, Examples, and Kappas for Categories of the DPICS

\begin{tabular}{|c|c|}
\hline Behavior Category & Definition \\
\hline \multicolumn{2}{|l|}{ Do Skills: } \\
\hline -Labeled Praise & $\begin{array}{l}\text { A specific statement of } \\
\text { approval and teaches the child } \\
\text { what can be done to receive } \\
\text { further parental approval; } \\
\text { "Contingent" if occurs within } 5 \\
\text { seconds of child compliance }\end{array}$ \\
\hline -Reflection & $\begin{array}{c}\text { A declarative statement that has } \\
\text { the same meaning as a child's } \\
\text { verbalization }\end{array}$ \\
\hline $\begin{array}{l}\text {-Behavioral } \\
\text { Description }\end{array}$ & $\begin{array}{l}\text { A non-evaluative, declarative } \\
\text { statement in which the subject } \\
\text { is the child and the verb is an } \\
\text { verbal or nonverbal action the } \\
\text { child has performed within the } \\
\text { last } 5 \text { seconds }\end{array}$ \\
\hline
\end{tabular}

“Avoid" Skills:

-Direct Command

A statement that contains an

"You did a great job of

$.49-.68$

building the tower."

Child says, "The choo-

$.46-.58$

choo go whiz," and

parent says, "The train

goes very fast."

"You drew a heart," or

$.46-.55$

"You're coloring the tree." order or direction for a behavior from the child

-Indirect Command

A suggestion for a behavior from the child that is implied or in question form

"Let's build a tower,"

$.63-.64$ or "Why don't you put your hands in your lap."

-Question

Verbal inquiries that have rising inflection and/ or

"What is this called?"

$.57-.80$ sentence structure of a question

-Negative Talk

A verbal expression of disapproval and includes sassy, sarcastic, rude, or impudent comments or "That's the red one, right?"

"You're being

$.65-.69$ naughty," or "Put it down or else!"

Other category:

-Child Compliance

Child performs, begins to $.69-.71$ perform, or attempts to perform

within 5 seconds a task requested by parent

Note: Definitions and examples from Eyberg et al., 2005, p. 46-136; Kappas from Brestan, Foote, \&

Eyberg, 2005, and Bessmer, Brestan, \& Eyberg, 2005, in Eyberg et al., 2005, p. 225-226 
Table 2.

Efficacy Studies of PCIT

\begin{tabular}{|c|c|c|c|c|c|}
\hline Authors & $\begin{array}{c}\text { Comparison } \\
\text { Group }\end{array}$ & $\begin{array}{l}\text { Therapist } \\
\text { Type }\end{array}$ & $\begin{array}{c}\text { Location of } \\
\text { Treatment }\end{array}$ & $\begin{array}{l}\text { Treatment } \\
\text { Integrity }\end{array}$ & Exclusion Criteria \\
\hline $\begin{array}{l}\text { Eyberg \& } \\
\text { Robinson } \\
(1982)\end{array}$ & None & $\begin{array}{l}\text { Psychology } \\
\text { Interns }\end{array}$ & $\begin{array}{l}\text { University- } \\
\text { based Clinic }\end{array}$ & $\begin{array}{c}\text { Not } \\
\text { Reported }\end{array}$ & $\begin{array}{l}\text { Child diagnosis of Mental } \\
\text { retardation, Autism, or } \\
\text { Schizophrenia }\end{array}$ \\
\hline $\begin{array}{l}\text { McNeil, } \\
\text { Eyberg, } \\
\text { Eisenstadt, } \\
\text { Newcomb, } \\
\& \\
\text { Funderburk } \\
(1991)\end{array}$ & $\begin{array}{c}\text { In } \\
\text { classroom: } \\
\text { No treatment } \\
\text { and } \\
\text { Typically } \\
\text { behaved }\end{array}$ & $\begin{array}{l}\text { Psychology } \\
\text { Interns }\end{array}$ & $\begin{array}{l}\text { University- } \\
\text { based Clinic }\end{array}$ & $\begin{array}{c}\text { Not } \\
\text { Reported }\end{array}$ & $\begin{array}{l}\text { (a) Parent or child } \\
\text { "history of moderate to } \\
\text { profound mental } \\
\text { retardation;" (b) Child } \\
\text { took medication for } \\
\text { hyperactivity }\end{array}$ \\
\hline $\begin{array}{l}\text { Eisenstadt, } \\
\text { Eyberg, } \\
\text { McNeil, } \\
\text { Newcomb, } \\
\& \\
\text { Funderburk } \\
(1993)\end{array}$ & None & $\begin{array}{l}\text { Doctoral } \\
\text { students with } \\
\text { 1-year PCIT } \\
\text { training }\end{array}$ & $\begin{array}{l}\text { University- } \\
\text { based Clinic }\end{array}$ & $\begin{array}{c}\text { Not } \\
\text { Reported }\end{array}$ & $\begin{array}{l}\text { (a) Parent or child } \\
\text { "history of moderate to } \\
\text { profound mental } \\
\text { retardation" (b) child } \\
\text { receiving medication for } \\
\text { ADHD }\end{array}$ \\
\hline $\begin{array}{l}\text { Schuhmann, } \\
\text { Foote, } \\
\text { Eyberg, } \\
\text { Boggs, \& } \\
\text { Algina } \\
(1998)\end{array}$ & Waitlist & $\begin{array}{l}\text { Graduate } \\
\text { student } \\
\text { therapists }\end{array}$ & $\begin{array}{l}\text { University- } \\
\text { based Clinic }\end{array}$ & $97 \%$ & $\begin{array}{l}\text { Children with "severe } \\
\text { physical or mental } \\
\text { impairments (e.g., } \\
\text { deafness, blindness, } \\
\text { autism)" (p. 35) }\end{array}$ \\
\hline $\begin{array}{l}\text { Nixon, } \\
\text { Sweeney, } \\
\text { Erickson, \& } \\
\text { Touyz } \\
(2003)\end{array}$ & $\begin{array}{l}\text { Waitlist and } \\
\text { Abbreviated } \\
\text { treatment }\end{array}$ & $\begin{array}{l}\text { First author } \\
\text { while doctoral } \\
\text { student }\end{array}$ & Not reported & $99.6 \%$ & $\begin{array}{l}\text { Behavior problems related } \\
\text { to "organic pathology, } \\
\text { trauma, or history of } \\
\text { severe physical and } \\
\text { mental deficits and } \\
\text { receiving medication to } \\
\text { manage behavioral } \\
\text { difficulties." (p. } 252 \text { ) }\end{array}$ \\
\hline $\begin{array}{l}\text { McCabe \& } \\
\text { Yeh (2010) }\end{array}$ & Usual care & $\begin{array}{l}\text { PCIT: MSW } \\
\text { and PsyD } \\
\text { graduate } \\
\text { students; UC: } \\
\text { LCSW and } \\
\text { PhD clinical } \\
\text { psychologist }\end{array}$ & $\begin{array}{l}\text { Community } \\
\text { Clinic }\end{array}$ & $82 \%$ & $\begin{array}{l}\text { Participating in another } \\
\text { psychosocial treatment for } \\
\text { child behavior problems. }\end{array}$ \\
\hline
\end{tabular}


Table 3.

Demographic Variables and Child Behavior at the First Assessment by Group

\begin{tabular}{|c|c|c|}
\hline Variable & PCIT Group & UC Group \\
\hline \multicolumn{3}{|l|}{ Child } \\
\hline$-\operatorname{Sex}$ & Male $33 \% *$ & Male $80 \% *$ \\
\hline -Age & $5.04(1.22)$ years & $5.06(1.11)$ years \\
\hline \multirow[t]{3}{*}{-Race } & Caucasian 73\% & Caucasian $80 \%$ \\
\hline & Biracial 20\% & Biracial 13\% \\
\hline & African American 7\% & African American 7\% \\
\hline -Latino/ Hispanic & $13 \%$ & $0 \%$ \\
\hline -Disruptive Behavior Disorder & $60 \%$ & $60 \%$ \\
\hline -Autism Spectrum Disorder & $33 \%$ & $27 \%$ \\
\hline -Aggression & $9(4.25)$ & $10.93(4.95)$ \\
\hline -Medication & $7 \%$ & $33 \%$ \\
\hline \multicolumn{3}{|l|}{ Caregiver } \\
\hline$-\operatorname{Sex}$ & Female $93 \%$ & Female $100 \%$ \\
\hline -Relation to Child & Biological Mother 93\% & Biological Mother $87 \%$ \\
\hline -Age & $31.87(4.79)^{*}$ & $27.83(2.78)^{*}$ \\
\hline -Race & Caucasian $100 \%$ & Caucasian $93 \%$ \\
\hline \multirow[t]{5}{*}{-Marital Status } & Married 33\% & Married $40 \%$ \\
\hline & Living with Partner 27\% & Living with Partner $13 \%$ \\
\hline & Separated $7 \%$ & Separated $7 \%$ \\
\hline & Divorced $20 \%$ & Divorced $7 \%$ \\
\hline & Never Married 13\% & Never Married 33\% \\
\hline \multirow[t]{4}{*}{-Employment Status } & Working Full Time 13\% & Working Full Time 13\% \\
\hline & Working Part Time $27 \%$ & Working Part Time $0 \%$ \\
\hline & Not Employed/ Seeking 13\% & Not Employed/ Seeking 33\% \\
\hline & $\begin{array}{c}\text { Not Employed/ Not Seeking 40\% } \\
\text { Other } 13 \%\end{array}$ & $\begin{array}{c}\text { Not Employed/ Not Seeking } 20 \% \\
\text { Other }^{1} 33 \%\end{array}$ \\
\hline \multirow[t]{6}{*}{-Highest Education } & Less than $9^{\text {th }}$ Grade $0 \%$ & Less than $9^{\text {th }}$ Grade $7 \%$ \\
\hline & Some High School 7\% & Some High School 13\% \\
\hline & $\begin{array}{l}\text { High School Diploma/ Equivalent } \\
27 \%\end{array}$ & $\begin{array}{l}\text { High School Diploma/ Equivalent } \\
40 \%\end{array}$ \\
\hline & Some College 33\% & Some College $20 \%$ \\
\hline & Associate's Degree 27\% & Associate's Degree 20\% \\
\hline & Bachelor's Degree 7\% & Bachelor's Degree 0\% \\
\hline -History of Counseling & $73 \%$ & $33 \%$ \\
\hline -Involvement with CPS & $55 \%$ & $36 \%$ \\
\hline \multicolumn{3}{|l|}{ Household } \\
\hline -Individuals Living in Home & $5(1.77)$ & $4.07(1.39)$ \\
\hline -Income & Median $\$ 22,000$ & $\$ 19,380$ \\
\hline
\end{tabular}

${ }^{1}$ Other $=$ retired, student, receiving Supplemental Security Income

$* p<.05$ 
Table 4.

Individual Participants' Demographic Information, Diagnoses, Dose, and Outcomes Ranked in Order of Greatest to Least Change on ECBI

\begin{tabular}{|c|c|c|c|c|c|c|c|c|c|c|c|c|c|}
\hline \multirow[b]{2}{*}{ Rank } & \multirow[b]{2}{*}{ Group } & \multirow{2}{*}{$\begin{array}{c}\text { Age \& } \\
\text { Sex } \\
\text { Child, } \\
\text { Parent } \\
\end{array}$} & \multirow{2}{*}{$\begin{array}{l}\text { Diag- } \\
\text { nosis }\end{array}$} & \multirow[b]{2}{*}{ Outcome } & \multicolumn{5}{|c|}{ Dose and Type of Therapy } & \multicolumn{2}{|c|}{ ECBI } & \multicolumn{2}{|c|}{$\begin{array}{l}\text { Comp- } \\
\text { liance }\end{array}$} \\
\hline & & & & & PCIT & $\mathrm{OP}$ & TSS & $\mathrm{BSC}$ & MT & 1 & 2 & 1 & 2 \\
\hline 1 & PCIT & $\begin{array}{l}4.55 \mathrm{~m} \\
\mathrm{NR} \mathrm{f}\end{array}$ & ADHD & $\begin{array}{l}\mathrm{CH} \text { : other } \\
\text { OP and } \\
\text { med (CDI) }\end{array}$ & 1 & $\begin{array}{c}\text { E } 5 \\
+ \\
\text { med }\end{array}$ & & & & 217 & 88* & .13 & .62 \\
\hline 2 & PCIT & $\begin{array}{l}5.46 \mathrm{~m}, \\
31.24 \mathrm{f}\end{array}$ & $\begin{array}{l}\text { Asp- } \\
\text { erger }\end{array}$ & Completed & 14 & & & & & 189 & $100 *$ & .56 & .72 \\
\hline 3 & PCIT & $\begin{array}{l}5.18 \mathrm{~m} \\
33.45 \mathrm{f}\end{array}$ & $\begin{array}{l}\text { Adjust } \\
\text { ment }\end{array}$ & Completed & 11 & & & & & 160 & $92 *$ & .07 & .50 \\
\hline 4 & PCIT & $\begin{array}{c}5.97 \mathrm{~m} \\
28.57 \mathrm{~m}\end{array}$ & DBD & Completed & 12 & & & & & 108 & 52 & .50 & .64 \\
\hline 5 & $\mathrm{UC}$ & $\begin{array}{l}4.13 \mathrm{~m}, \\
29.72 \mathrm{f}\end{array}$ & DBD & Drop Out & & & 0 & 0 & 0 & 193 & 140 & .40 & .15 \\
\hline 6 & UC & $\begin{array}{l}4.32 \mathrm{f}, \mathrm{b} \\
29.71 \mathrm{f}\end{array}$ & DBD & Completed & $(\mathrm{F}$ & B & M & $\mathrm{H}$ & S) & 157 & $116^{*}$ & .43 & .41 \\
\hline 7 & UC & $\begin{array}{l}7.01 \mathrm{~m}, \\
\mathrm{NR} \mathrm{f}\end{array}$ & Bipolar & $\begin{array}{l}\text { CH: other } \\
\text { OP }\end{array}$ & & E 7 & 39.25 & 26.25 & 3 & 108 & 76 & .56 & .38 \\
\hline 8 & $\mathrm{UC}$ & $\begin{array}{l}6.13 \mathrm{~m}, \\
28.32 \mathrm{f}\end{array}$ & ADHD & Continuing & & & 280.75 & 41 & 0 & 216 & 185 & .12 & .41 \\
\hline 9 & PCIT & $\begin{array}{l}5.56 \mathrm{~m}, \\
29.30 \mathrm{f}\end{array}$ & DBD & $\begin{array}{l}\text { CH: other } \\
\text { OP (PDI) }\end{array}$ & 12 & $\begin{array}{c}\mathrm{E} \\
12\end{array}$ & & & & 184 & 159 & .44 & .58 \\
\hline 10 & PCIT & $\begin{array}{l}3.50 \mathrm{f} \\
23.37 \mathrm{f}\end{array}$ & $\begin{array}{l}\text { ASD, } \\
\text { DBD }\end{array}$ & $\begin{array}{l}\text { CH: BHRS } \\
\text { (PDI) }\end{array}$ & 10 & & E 120 & E 60 & E 0 & 147 & $122 *$ & .06 & .17 \\
\hline 11 & PCIT & $\begin{array}{l}6.21 \mathrm{f} \\
32.44 \mathrm{f}\end{array}$ & ADHD & $\begin{array}{c}\text { BHRS } \\
\text { Continuing } \\
\text { (PDI) }\end{array}$ & 8 & & E 140 & E 60 & E 0 & 190 & 165 & .57 & .50 \\
\hline 12 & PCIT & $\begin{array}{l}5.01 \mathrm{~m}, \\
35.71 \mathrm{f}\end{array}$ & DBD & Continuing & E 17 & & & & & 146 & $123 *$ & .20 & .51 \\
\hline 13 & UC & $\begin{array}{l}4.67 \mathrm{~m}, \\
30.69 \mathrm{f}\end{array}$ & ASD & Completed & & & 245.50 & 93.25 & E 0 & 139 & $129 *$ & .38 & .88 \\
\hline 14 & UC & $\begin{array}{l}3.84 \mathrm{f} \\
22.34 \mathrm{f}\end{array}$ & ASD & Continuing & & & E 384 & E 30 & 0 & 154 & 145 & .14 & .19 \\
\hline 15 & UC & $\begin{array}{l}3.85 \mathrm{~m}, \\
27.12 \mathrm{f}\end{array}$ & ASD & Continuing & & & 116 & 299.75 & 0 & 142 & 142 & .09 & .30 \\
\hline 16 & UC & $\begin{array}{l}5.00 \mathrm{~m}, \\
25.30 \mathrm{f}\end{array}$ & DBD & Continuing & & & 114.75 & 43.75 & 0 & 48 & 49 & .26 & .27 \\
\hline 17 & UC & $\begin{array}{l}5.85 \mathrm{~m}, \\
27.85 \mathrm{f}\end{array}$ & ADHD & Drop Out & & & 28.5 & 17 & 0 & 177 & 180 & .20 & .54 \\
\hline 18 & $\mathrm{UC}$ & $\begin{array}{l}7.05 \mathrm{~m}, \\
31.24 \mathrm{f}\end{array}$ & ADHD & Continuing & & & 227 & 119 & 12 & 168 & 171 & .20 & .20 \\
\hline 19 & PCIT & 3.99f, & ASD & CH: BHRS & E 9 & & E 210 & E 84 & E 0 & 122 & 127 & .00 & .12 \\
\hline
\end{tabular}


30.66f (PDI)

\begin{tabular}{|c|c|c|c|c|c|c|c|c|c|c|c|}
\hline 20 & $\mathrm{UC}$ & $\begin{array}{l}4.29 \mathrm{~m} \\
26.03 \mathrm{f}\end{array}$ & $\begin{array}{l}\text { Asp- } \\
\text { erger }\end{array}$ & Drop Out & & 34.75 & 15.75 & 0 & 87 & 92 & .27 \\
\hline 21 & PCIT & $\begin{array}{l}7.21 \mathrm{f} \\
36.16 \mathrm{f}\end{array}$ & $\begin{array}{l}\text { ASD } \\
\text { DBD }\end{array}$ & Continuing & 17 & & & & 95 & 104 & .21 \\
\hline 22 & PCIT & $\begin{array}{l}5.36 \mathrm{f} \\
32.85 \mathrm{f}\end{array}$ & $\begin{array}{l}\text { DBD, } \\
\text { Trauma }\end{array}$ & $\begin{array}{l}\text { Drop Out } \\
\text { (CDI) }\end{array}$ & 1 & & & & 106 & 121 & .04 \\
\hline 23 & UC & $\begin{array}{l}5.66 \mathrm{~m}, \\
30.04 \mathrm{f}\end{array}$ & ADHD & Continuing & & E 195 & E 91 & $\begin{array}{c}E \\
26\end{array}$ & 223 & 243 & .52 \\
\hline 24 & UC & $\begin{array}{c}4.55 \mathrm{~m} \\
\mathrm{NR} \mathrm{f}\end{array}$ & ADHD & $\begin{array}{l}\text { Drop Out } \\
\text { (Home } \\
\text { Hours) }\end{array}$ & & 28 & 18 & 0 & 148 & 169 & .16 \\
\hline 25 & PCIT & $\begin{array}{l}3.30 \mathrm{f} \\
40.53 \mathrm{f}\end{array}$ & $\begin{array}{l}\text { ASD, } \\
\text { DBD }\end{array}$ & $\begin{array}{c}\text { BHRS } \\
\text { Continuing } \\
\text { (PDI) }\end{array}$ & 16 & E 128 & E 32 & 0 & 136 & 163 & .13 \\
\hline
\end{tabular}

Did not complete second assessment

\begin{tabular}{|c|c|c|c|c|c|c|c|c|c|c|c|c|c|}
\hline & $\mathrm{UC}$ & $\begin{array}{l}3.82 \mathrm{f} \\
23.69 \mathrm{f}\end{array}$ & DBD & Foster Care & & & 27.25 & 5.75 & 0 & 214 & -- & .55 & -- \\
\hline & PCIT & $\begin{array}{l}5.97 \mathrm{~m} \\
28.57 \mathrm{f}\end{array}$ & $\begin{array}{l}\text { DBD, } \\
\text { ASD }\end{array}$ & $\begin{array}{l}\text { Drop Out } \\
\text { (CDI) }\end{array}$ & 4 & & & & & 187 & -- & .25 & -- \\
\hline & UC & $\begin{array}{l}5.80 \mathrm{~m}, \\
29.72 \mathrm{f}\end{array}$ & $\begin{array}{c}\text { ADHD, } \\
\text { Bipolar, } \\
\text { ASD }\end{array}$ & Continuing & & & 290.25 & 42.76 & 12 & 177 & -- & .28 & -- \\
\hline & PCIT & $\begin{array}{l}2.74 \mathrm{f} \\
29.96 \mathrm{f}\end{array}$ & DBD & $\begin{array}{l}\text { Drop Out } \\
\text { (PDI) }\end{array}$ & 9 & & & & & 111 & -- & .50 & -- \\
\hline & PCIT & $\begin{array}{l}4.58 \mathrm{f} \\
26.62 \mathrm{f}\end{array}$ & $\begin{array}{c}\text { Adjust } \\
\text { ment } \\
\text { Trauma }\end{array}$ & $\begin{array}{c}\text { Drop Out } \\
\text { (CDI) }\end{array}$ & 1 & & & & & 88 & -- & .19 & -- \\
\hline & & & & & & & & & & & & $\begin{array}{l}\text { Cor } \\
\text { lian }\end{array}$ & \\
\hline Rank & Group & Parent & nosis & Outcome & PCIT & OP & TSS & BSC & MT & 1 & 2 & 1 & 2 \\
\hline
\end{tabular}

*Change from Clinically Significant to Normative Range; Bold indicates improvement on both ECBI and Compliance PCIT $=$ Parent-Child Interaction Therapy; $\mathrm{UC}=$ Usual Care; $\mathrm{OP}=$ Other Outpatient therapy; FBMHS $=$ Family-based mental health services; BHRS $=$ Behavioral Health Rehabilitative Services; NR $=$ Not Reported; $m=$ male; $f=$ female; age in years; DBD = Disruptive Behavior Disorder; ASD = Autism Spectrum Disorder; ADHD = Attention Deficit/ Hyperactivity Disorder; $\mathrm{CH}=$ change to the type of therapy indicated after the colon; Continuing = at the second assessment that family was continuing with same type of therapy as at the first assessment; TSS = Therapeutic Support Staff; BSC = Behavior Specialist Consultant; MT = Mobile Therapist; E = Estimation of dose is based on parent report because therapist report unavailable; Med = child began medication management; Phase in which discontinued PCIT is indicated in parentheses 
Table 5.

Description of Treatment and Fidelity

\begin{tabular}{|c|c|c|c|c|}
\hline & PCIT Manual & $\begin{array}{l}\text { CDI Coaching } \\
\text { Session } \\
\end{array}$ & $\begin{array}{c}\text { PDI Coaching } \\
\text { Session }\end{array}$ & $\begin{array}{c}\text { UC "Typical” } \\
\text { Week }\end{array}$ \\
\hline Sample Size & & 31 & 22 & 11 \\
\hline $\begin{array}{l}\text { Administer } \\
\text { standardized } \\
\text { parent-report } \\
\text { measure }\end{array}$ & Weekly & $74.2 \%$ & $95.5 \%$ & $0 \%$ \\
\hline $\begin{array}{l}\text { Review changes } \\
\text { on standardized } \\
\text { parent report } \\
\text { measure }\end{array}$ & Weekly & $32.3 \%$ & $22.7 \%$ & $0 \%$ \\
\hline $\begin{array}{l}\text { Spend a "few } \\
\text { minutes" talking } \\
\text { with parent }\end{array}$ & Weekly & $\begin{array}{l}20.32 \text { minutes } \\
(\mathrm{SD}=9.99)\end{array}$ & $\begin{array}{l}23.41 \text { minutes } \\
(\mathrm{SD}=7.62)\end{array}$ & $\begin{array}{l}9.25 \text { minutes } \\
(\mathrm{SD}=20.21)\end{array}$ \\
\hline Give homework & Weekly & $93.5 \%$ & $100 \%$ & $15.8 \%$ \\
\hline $\begin{array}{l}\text { Collect } \\
\text { homework }\end{array}$ & Weekly & $70 \%$ & $77.3 \%$ & $4.8 \%$ \\
\hline $\begin{array}{l}\text { Spend time } \\
\text { individually with } \\
\text { child }\end{array}$ & None of sessions & $\begin{array}{c}3.2 \% \\
\text { (unknown } \\
\text { length*) }\end{array}$ & $0 \%$ & $\begin{array}{c}27.27 \% \\
18.75 \text { minutes } \\
(\mathrm{SD}=32.87)\end{array}$ \\
\hline $\begin{array}{l}\text { Code parent } \\
\text { behavior }\end{array}$ & $\begin{array}{l}\text { Weekly, except } \\
\text { for } 1^{\text {st }} \text { PDI } \\
\text { session }\end{array}$ & $93.5 \%$ & $68.2 \%$ & $9.5 \%$ \\
\hline $\begin{array}{l}\text { Code child } \\
\text { behavior }\end{array}$ & $\begin{array}{l}\text { Weekly in PDI } \\
\text { only, except } 1^{\text {st }} \\
\text { PDI session }\end{array}$ & Not applicable & $68.2 \%$ & $42.9 \%$ \\
\hline $\begin{array}{l}\text { Give feedback } \\
\text { on parent and/ or } \\
\text { child behavior }\end{array}$ & Weekly & $45.2 \%$ & $9.1 \%$ & $4.8 \%$ \\
\hline $\begin{array}{l}\text { Coach parent in } \\
\text { interactions with } \\
\text { child }\end{array}$ & $15-25$ minutes & $\begin{array}{c}100 \% \\
28.23 \text { minutes } \\
(\mathrm{SD}=9.88) \\
\end{array}$ & $\begin{array}{c}100 \% \\
35.86 \text { minutes } \\
(\mathrm{SD}=20.66) \\
\end{array}$ & $\begin{array}{c}81 \% \text { (unknown } \\
\text { length**) }\end{array}$ \\
\hline
\end{tabular}


Table 6.

Responses on the Qualitative Caregiver Satisfaction Survey

PCIT UC (BHRS and FBMHS)
What did you like about the services you received for your child?

1. Made aware of different techniques for handling behaviors, talking techniques, techniques that are very efficient and how good results. Showed me things to change and not do that I used to do.

2. Like Therapist. She is very into her job and is effective. I like the program, and its approach and tactics.

3. I liked that we could make it fit our home life. Also that we could implement with younger siblings.

4. I like that the therapist uses the parentchild relationship to set the basis for treatment.

5. Explained things, more controlled environment, helpful

6. Patient, good child therapist

7. It helped me to take the time more often to have "special play time" which helped his behavior because I was giving him more attention.

8. Helpful in understanding child more and her needs; I'm still learning; therapist directs me and tells me how I could do differently.

9. I liked the skills about how to address his behaviors and discipline.

10. One-on-one attention

11. Good ideas

12. (Dropped out in CDI and received medication) When she got medicated it helped her be able to focus more in school and it has calmed her down.
1. The support of some of the staff while they were here.

2. Helped me understand my child and what he was going through and how to deal with it

3. Helped me understand what works for my child, and how to help him. Helped him learn to help himself.

4. I liked that my child's workers are very involved with his needs and that they also are so eager to help our other family members with their concerns.

5. Very supportive and answered all my questions

6. I like the skills they are teaching her, especially calming herself down.

7. None

8. It told me that my kid can be responsible

9. I like that it is in the house and it is one-onone

10. New ideas of things to do as a family and one-on-one with child

11. Coming to home helped with homework when he wouldn't do it with me.

12. (Transferred from BHRS to Outpatient Therapy) Working wonderful, I love it. I'm putting my foot down, not giving in.

13. $(F B M H S)$ They were very patient with me and my child. They were hands on, on teaching me and helping me through punishment and better behavior.

14. (Transferred from PCIT to BHRS) Show me how to work with my kids, how involved and how much they care

15. (Transfer from PCIT to BHRS) They cope with her behaviors

What did you not like about the services you received for your child?

1. In the beginning (maybe week 4-5) it felt like we were never going to see results. I almost dropped out, but then our therapist moved us to the next phase even though I was close to hitting the mark but not quite there.

2. The way she wanted me to do time-out with him because I have five other kids. She wanted me to watch him.

3. Felt that the behavior was not explained properly and medication was the first step in "fixing" problems. Was told my child was slow and behind. I had him tested and
1. The constant personnel changes. The different programs started but not followed through with.

2. It took awhile to find a match for my child and someone he can trust and liked

3. The therapists mostly followed my lead rather than instructing me if he was having issues. It is very dependent on having a good dedicated therapist and behavioral specialist.

4. Don't feel like they give him a chance. They can't relate to us. She's pushing me, and I don't have a choice. 
he is advanced. He is getting A's in school.

4. The time it took to the second phase

5. Automatically expect child to know self play, work on self play first.
5. The travel for the staff to drive to come and see our family

6. Not a lot of change to treatment plans

7. Gave up on redirecting in the end.

8. (Did not initiate BHRS or outpatient therapy after insurance dispute) My child didn't enroll in services due to staff issues and mom's school schedule. The fact that we would go sit in an office that was set up for my child. So my child wouldn't show the same behaviors as he would in his home setting.

9. (Transferred from BHRS to outpatient therapy) Not fond of missing school every other week.

10. (Transferred from PCIT) How many hours they're in my home and how long it seems to be taking for everything to come together

11. (Transferred from PCIT) Not consistent on times of day they are supposed to be here, some didn't do their job, just sat for two hours, late and excuses for everything

\section{What was most useful for helping your child?}

1. To get her away from whining, and just to talk and ask. Techniques for time-out, how to talk in more effective ways and getting better results in return. (I don't care who anyone is or what they know. There are always things to learn and new things to try.)

2. The "Special" playtime and then getting her respect from my commands.

3. Setting up house rules and using time-out. He knew he had one warning.

4. Learning how to communicate effectively with my child. Creating a closeness with my child through play.

5. Really helped with her screaming, starting to help with her behavior, take the time to do what she wanted to do, connect with her

6. Teaching me how to talk to him. I don't always use it, but it can be useful.

7. It helped me to discipline without getting stressed from repeating myself constantly. The praising and positive attitude and comments made during special playtime helped to make him feel proud and happy. All the tools I learned only helped.

8. Directional play

9. How to approach my child (e.g., do not use "no," "don't," "stop," "quit"). Sticking to my rules. Be consistent.

10. One-on-one attention

11. Take playtime everyday; sometimes we're
1. Some one on one time. The little of talking about emotions he received.

2. Learning that he is not alone and there are people here to help him

3. Visual schedule helps with transitioning. Discipline to sit and do work prepared him well for kindergarten.

4. Trying to make him listen.

5. Repeated tasks and positive reinforcement offered to my child. Also, keeping a routine with a visual schedule.

6. All the valuable information

7. When she first learned to sign, now she is using words and improving a lot.

8. I spent more time using positive reinforcers and rewarded good behaviors.

9. He learned his manners

10. His talking, how he acts

11. Showing him how to work better with others

12. Not useful

13. (Transferred to Outpatient Therapy) Taught him coping skills. He's on a behavior chart. I'm putting my foot down.

1. $(F B M H S)$ The feelings cards and the proper time out techniques. The way they helped me handle her behavior. They were amazing at their job.

14. (Transferred from PCIT) When they are here and she has a behavior, they can show me what to do. 
so busy we don't' get to take that time.

12. (CDI drop out and received medication)

Has someone that she can talk to when she is upset now (therapist $1 \mathrm{x}$ per month)
15. (Transferred from PCIT) Nothing really

What did you think was not useful in helping your child?

1. That she didn't have to clean up her toys after Special Play Time. Cleanup is part of play. (Participant dropped out before $P D I)$.

2. Play time

3. The need to immediately medicate my child before more trying other methods. Thought the problems were much worse than really were.

4. (Transferred to BHRS) First phase (trust)she already knew she could trust us.

Having the child change behavior that they can't help because they see it differently. I didn't see the use in triggering a tantrum over something unimportant (e.g., noncompliance with "give me the toy" in your hand) when I deal with many tantrums all day.

5. (Transferred to BHRS) Needed someone in the home on regular basis.
2. Making him feel everyone will leave, since there have been so many changes in staff.

3. Other/ past workers the let him over run them to where he was always in control

4. Sometimes I think he was overwhelmed by too much work (when school and therapy added)

5. When he didn't listen, it was hard to get him back into the swing.

6. Daily program- not real life

7. He had this attitude that he didn't have to listen to me; he only had to listen to them. Them "babyfying" him. Them being out here all the time (four nights per week). I couldn't handle it. It was very overwhelming. I felt like I didn't have a life.

8. (Did not initiate BHRS or outpatient therapy after insurance dispute) Doing outpatient and the fact is I didn't need help communicating with my child.

9. (Transferred from PCIT) Not helping with her behaviors to expectations, they come and did stuff I could do or already do with her. Not new ideas.

Would you recommend the type of services that you received to another family that was

\begin{tabular}{|c|c|}
\hline & \\
\hline $\begin{array}{l}\text { O "Definitely Not" } \\
\text { 1"Mavbe" }\end{array}$ & $\begin{array}{c}1 \text { "Definitely Not" } \\
4 \text { "Mavbe" }\end{array}$ \\
\hline $\begin{array}{c}1 \text { "Maybe" } \\
4 \text { "Yes" }\end{array}$ & $\begin{array}{c}4 \text { "Maybe" } \\
\text { 0"Yes" }\end{array}$ \\
\hline 7 "Definitely Yes" & 8 "Definitely Yes" \\
\hline
\end{tabular}

Note: The five participants in the UC group who reported Definitely Not or Maybe had not been diagnosed with an ASD. The one participant in the PCIT group who reported Maybe had not been diagnosed with an ASD. 
Table 7.

PCIT Therapists' Responses on Qualitative Survey

\begin{tabular}{l}
\hline Question \\
\hline With how many \\
cases in total \\
have you \\
completed \\
PCIT? \\
With how many \\
cases in total \\
have you \\
attempted \\
PCIT?
\end{tabular}

With how many cases are you in the process of providing PCIT?

What do you like about PCIT?

What do you not like about PCIT?

What was most useful about
Answer

$6,16,6$, more than $35,20,12$

$1,4,0,11,8,1$

1. It is effective. It targets the real problem-which isn't the child. It is a nonthreatening way to work with parents who lack parenting skills.

2. PCIT is very structured and focuses on the strengths to lead into the discipline. I enjoy the play therapy and the fact parents have to play with their children in session. The design of the one-way mirror really benefits the program in allowing the parent and child to engage with each other and build on their relationship. The piece that I love is the enjoyment parents gain from playing with their children. Initially most parents do not enjoy playing with their children because of behaviors and after CDI parents begin to enjoy playing again.

3. Real time, hands on practical approach. Data-based and provision of more objective parent report.

4. Structure, parental expectations, coaching, constant assessment of progress/ regression

5. User-friendly, coaching, it works.

6. Effectiveness of giving parents skills to manage problem behaviors; empowering families.

1. Don't like technical problems with ear buds, not being able to see what is going on very well, camera "floating", etc. I tend to just stay in the room and coach CDI for that reason

2. The structure can be a barrier at times. Because PCIT focuses on behavior modification many times children with trauma will begin to process trauma after the $4^{\text {th }}$ visit and then it delays PCIT.

3. It does not provide for an emotional outlet nor does it address underlying emotional issues that may influence behaviors.

4. Not much time to process other family concerns, parental stress. Difficult to meet the needs of varying parental learning styles.

5. Time-consuming paperwork

6. Difficulty in finding families who are stable enough to commit to treatment.

1. CDI skills.

2. PCIT has built on attachment and engagement. To watch the parents/child 
PCIT in helping your clients?

What parts of PCIT did you not think were useful in helping your clients?

Did you often add any therapeutic practices to your treatment that are not included in PCIT (e.g., token economy, school-home note)? If so, what practices and why?

What aspects of PCIT were difficult to implement and why?

Will you continue using PCIT in clinical practice? relationship grow in priceless. With children in the spectrum it is amazing to watch their verbal skills and comprehension skills develop.

3. Accountability provided through weekly homework review, demonstrating (through ECBI scores) progress on a weekly basis.

4. On site coaching

5. New Skills and coaching

6. Manage child behaviors.

1. I find that after graduating them, the parents fall back into "default" mode parenting if not seen regularly. I wish it were true that we could teach these skills and then therapy would be over, but it is not.

2. Nothing

3. I can't think of anything!

4. ECBI at every session. Not time to provide processing of other relevant problems.

5. No response.

6. N/A

1. I have had to add the elements of Didactic Developmental Psychotherapy (PACE skills) with a child whose trauma symptoms were so activated when he came to some sessions that he would not play. He was just highly emotional.

2. No I do not add interventions or practices. If other things such as trauma arise I will stop and address the prominent concern and then pick up with PCIT.

3. No

4. No, not during active treatment usually on one occasion, I was working with an elderly grandparent who could NOT physically redirect the child. The therapist offered reward if the grandmother reported that the child sat in the time out chair.

5. Token economy; home-school note to track progress at school/ daycare

6. Relationship-based interventions; psycho education on normal child development and expectations for child behaviors

1. PDI is more difficult for me because in-home services are not consistent with what I am teaching and the parents get confused about what they should be doing.

2. PDI was difficult initially but after a few clients have went through and participate in supervision it builds your confidence as a therapist it became easier.

3. There are many external barriers that make the model difficult to implement. The two primary are inconsistencies with transportation and attendance and MCO restrictions on PCI while BHRS is in place.

4. Gaining generalization of skills, maintaining parent motivation, when PCIT was being implemented with BHRS services.

5. Homework- follow through is sporadic; Difficulty depends on parents' mental health issues, family crisis level, and poor coping skills. Grandparents are hard. PDI Homework.

6. Not having the time to support families when experiencing other challenges.

1. Yes

2. Absolutely! I hope!

3. Yes

4. Yes

5. Yes 


\section{Yes}

$\begin{array}{lll}\text { Would you } & \text { 1. } & \text { Yes } \\ \text { recommend that } & \text { 2. } & \text { Yes. } \\ \text { a colleague } & \text { 3. } & \text { Yes } \\ \text { receive training } & \text { 4. } & \text { Yes } \\ \text { in PCIT? } & \text { 5. Yes } \\ & 6 . \quad \text { Yes }\end{array}$

What

1. Homework, coding, and ECBI.

components of

2. All

PCIT did you

3. All aspects

4. No Response

almost always

use (e.g.,

coding, house

5. Coding, ECBI, House Rules

6. Followed by manual

rules, public

behavior,

homework)?

What

components of

PCIT did you

often leave out

(e.g., coding,

house rules,

public behavior, homework)?

1. Never got to public behavior with my families due to interference from inhome services or parent not wanting to continue with PDI. Have serious difficulty coding PDI - can't observe, coach and write at same time!

2. Public behavior.

3. I only used house rules as needed. They aren't always needed.

4. ECBI at every session

5. No response

6. None 
Table 8

PCIT $(N=12)$ Compared to UC $(N=13)$

Test Statistics and p Values of the Interaction for Outcome Measures

\begin{tabular}{|c|c|c|c|c|c|c|c|c|}
\hline & & \multicolumn{2}{|c|}{ Assessment 1} & \multicolumn{2}{|c|}{ Assessment 2} & \multirow[b]{2}{*}{$\begin{array}{c}\text { Test } \\
\text { Statistic }\end{array}$} & \multirow[b]{2}{*}{$\begin{array}{c}p \\
\text { value }^{1}\end{array}$} & \multirow[b]{2}{*}{$\begin{array}{c}\text { Cohen's } \\
d^{2}\end{array}$} \\
\hline & & M & (SD) & M & (SD) & & & \\
\hline \multicolumn{9}{|l|}{ ECBI } \\
\hline \multirow[t]{2}{*}{ Intensity } & PCIT & 150.00 & (38.84) & 118 & $(33.71)$ & $F=2.44$ & .13 & .53 \\
\hline & $\mathrm{UC}$ & 150.77 & (49.13) & 141 & $(51.17)$ & & & \\
\hline \multicolumn{9}{|l|}{ CBCL } \\
\hline \multirow[t]{2}{*}{ External } & PCIT & 70.17 & (10.86) & 62.67 & (12.43) & $F=1.40$ & .25 & .34 \\
\hline & $\mathrm{UC}$ & 69.69 & (12.98) & 66.69 & (11.18) & & & \\
\hline \multicolumn{9}{|l|}{ CBCL } \\
\hline \multirow[t]{2}{*}{ Internal } & PCIT & 60.75 & $(5.94)$ & 58.50 & $(9.89)$ & $F=.00$ & 1.0 & .35 \\
\hline & $\mathrm{UC}$ & 64.77 & $(6.88)$ & 62.54 & (12.77) & & & \\
\hline \multirow[t]{2}{*}{ PSI Total } & PCIT & 90.67 & (21.66) & 89.67 & $(26.16)$ & $F=.25$ & .62 & .08 \\
\hline & $\mathrm{UC}$ & 92.69 & (19.49) & 87.62 & (24.16) & & & \\
\hline \multicolumn{9}{|l|}{ Positive } \\
\hline \multirow[t]{2}{*}{ Composite } & PCIT & 7.58 & $(6.14)$ & 8.5 & $(7.65)$ & $F=.13$ & .72 & 1.20 \\
\hline & UC & 2.00 & $(1.47)$ & 1.69 & $(2.36)$ & & & \\
\hline \multicolumn{9}{|l|}{ Negative } \\
\hline \multirow[t]{2}{*}{ Composite } & PCIT & 16.92 & (11.52) & 14.42 & $(10.56)$ & $F=.07$ & .79 & .70 \\
\hline & $\mathrm{UC}$ & 26.77 & (10.75) & 22.92 & (13.67) & & & \\
\hline \multirow[t]{2}{*}{ Percent DC } & PCIT & .62 & $(.16)$ & .47 & $(.20)$ & $F=3.31$ & .08 & -1.5 \\
\hline & $\mathrm{UC}$ & .78 & $(.08)$ & .74 & (.16) & & & \\
\hline \multirow[t]{2}{*}{ Compliance } & PCIT & .24 & $(.21)$ & .49 & $(.23)$ & $F=3.98$ & .06 & .64 \\
\hline & $\mathrm{UC}$ & .28 & $(.16)$ & .35 & $(.20)$ & & & \\
\hline \multicolumn{9}{|l|}{ LP } \\
\hline \multirow[t]{2}{*}{ Compliance } & PCIT & .00 & $(.00)$ & .67 & (1.37) & $X^{2}=3.69$ & .06 & .69 \\
\hline & $\mathrm{UC}$ & .08 & $(.28)$ & .00 & $(.00)$ & & & \\
\hline \multirow[t]{2}{*}{ TAI } & PCIT & & & 39.33 & $(6.96)$ & $t=.18$ & .86 & .07 \\
\hline & $\mathrm{UC}$ & & & 38.77 & $(8.27)$ & & & \\
\hline
\end{tabular}

PCIT $=$ Parent-Child Interaction Therapy; UC $=$ Usual Care; DC $=$ Direct Command; $\mathrm{LP}=$ Labeled Praise

PCIT group $\mathrm{n}=12$; $\mathrm{UC}$ group $\mathrm{n}=13$

${ }^{1}$ Given that 30 families enrolled and 25 families completed the second assessment, the study is underpowered and $p$ values should be interpreted cautiously.

${ }^{2}$ Cohen's $d$ is for scores between groups at the second assessment 
Table 9.

Cohen's d Effect Sizes from First to Second Assessment by Group and Comparison to Past Research

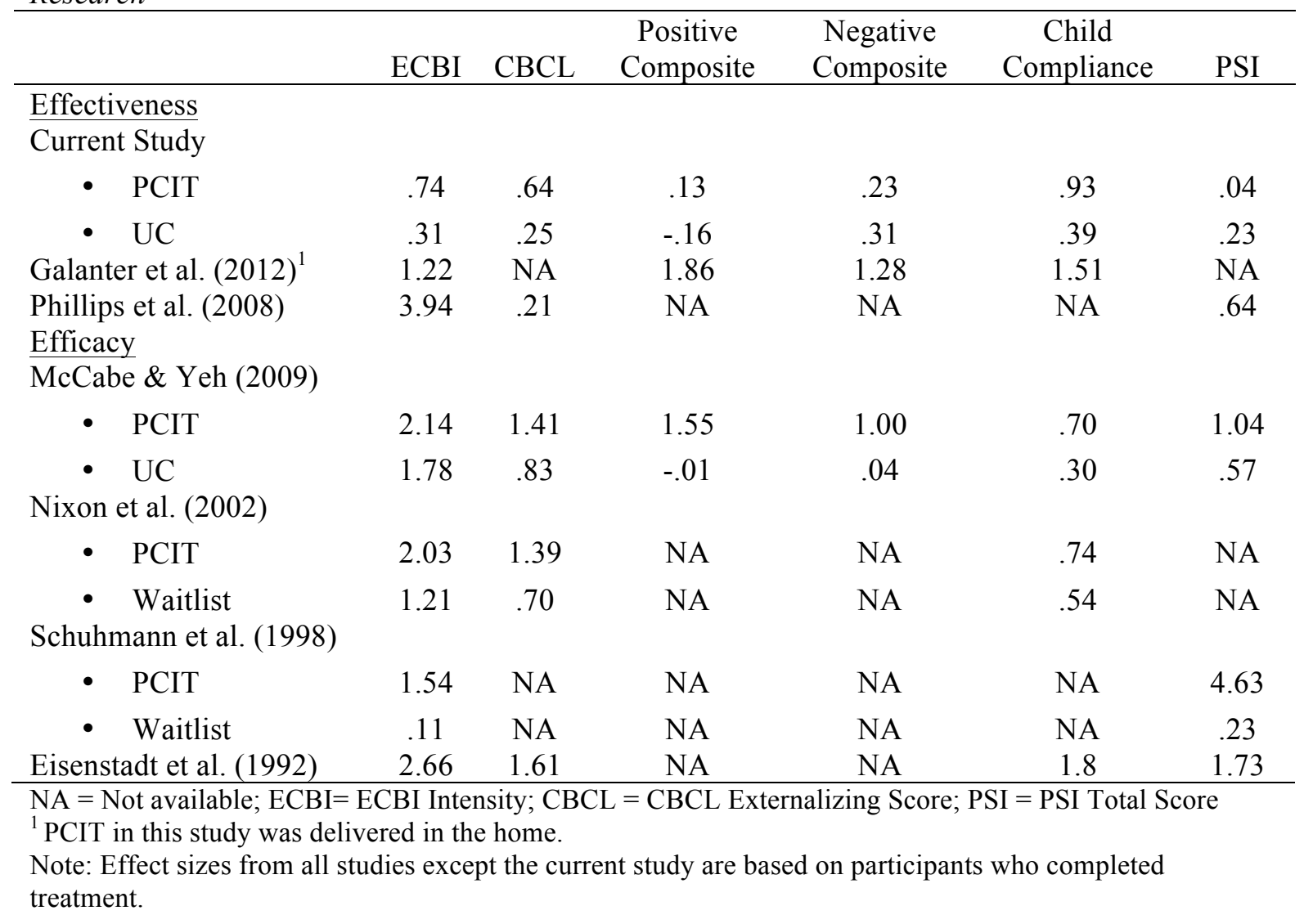


Table 10.

"Pure" PCIT $(N=6)$ and "Pure" BHRS $(N=10)$

Test Statistics and p Values of the Interaction for Outcome Measures

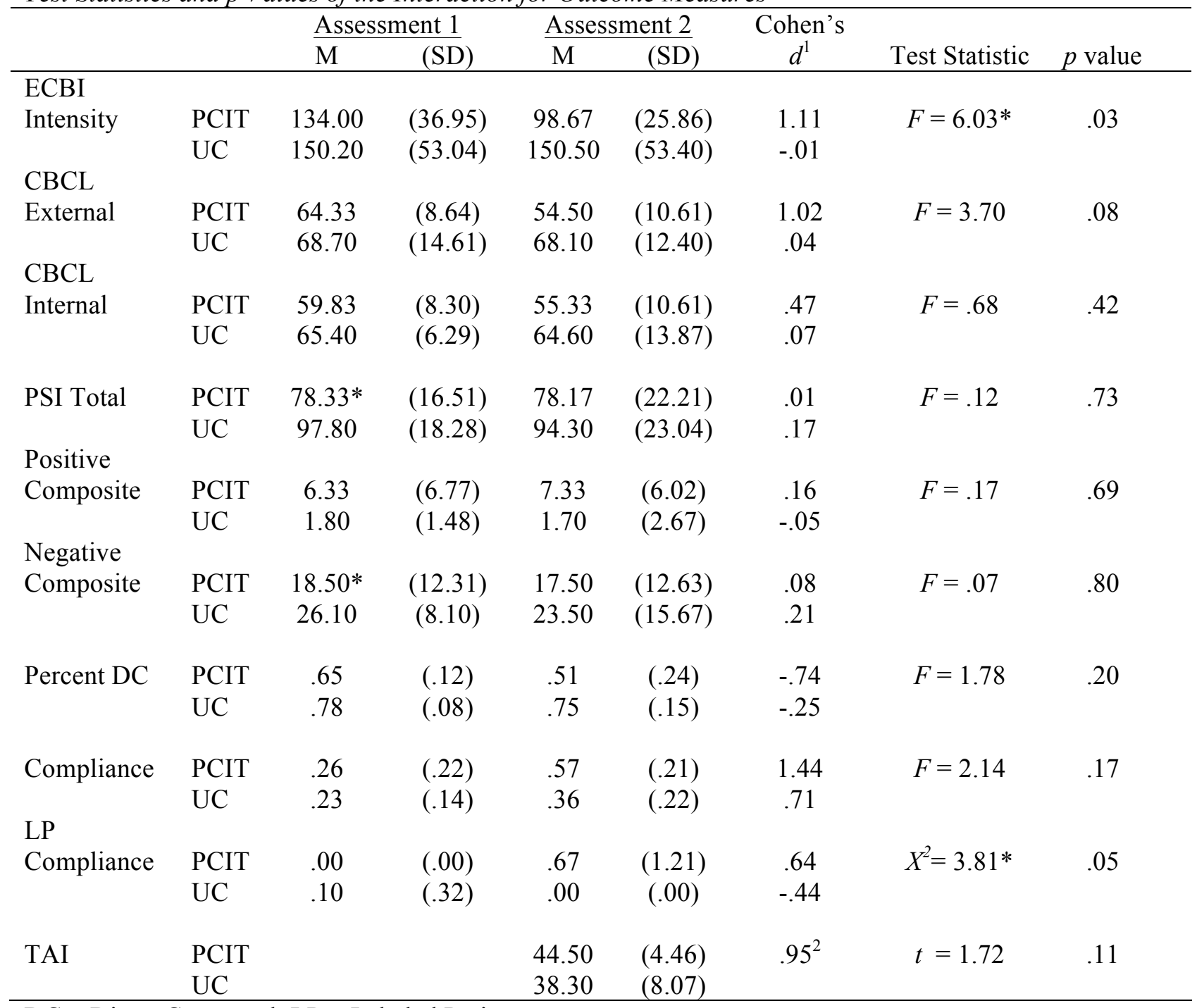

$\mathrm{DC}=$ Direct Command; LP $=$ Labeled Praise

${ }^{1}$ Cohen's $d$ represents the difference from the first to second assessment within each group.

${ }^{2}$ This effect size represents the difference between the two groups rather than the difference from the first to second assessment within each group.

Note: Families included in these analyses had completed PCIT or BHRS or were continuing with PCIT and BHRS. Families were not included if they received both PCIT and BHRS, transferred to another type of therapy during the study, did not receive either PCIT or BHRS, or dropped out of treatment. 
Table 11.

"Pure" PCIT $(N=6)$ and "Pure" BHRS $(N=10)$

Individual Participants' Demographic Information, Diagnoses, Dose, and Outcomes Ranked in Order of Greatest to Least Change on ECBI

\begin{tabular}{|c|c|c|c|c|c|c|c|c|c|c|c|c|c|}
\hline \multirow[b]{2}{*}{ Rank } & \multirow[b]{2}{*}{ Group } & \multirow{2}{*}{$\begin{array}{l}\text { Age \& } \\
\text { Sex } \\
\text { Child, } \\
\text { Parent } \\
\end{array}$} & \multirow{2}{*}{$\begin{array}{l}\text { Diag- } \\
\text { nosis }\end{array}$} & \multirow[b]{2}{*}{ Outcome } & \multicolumn{5}{|c|}{ Dose and Type of Therapy } & \multicolumn{2}{|c|}{ ECBI } & \multicolumn{2}{|c|}{$\begin{array}{l}\text { Comp- } \\
\text { liance }\end{array}$} \\
\hline & & & & & PCIT & $\mathrm{OP}$ & TSS & $\mathrm{BSC}$ & MT & 1 & 2 & 1 & 2 \\
\hline 1 & PCIT & $\begin{array}{l}5.46 \mathrm{~m} \\
31.24 \mathrm{f}\end{array}$ & $\begin{array}{l}\text { Asp- } \\
\text { erger }\end{array}$ & Completed & 14 & & & & & 189 & $100 *$ & .56 & .72 \\
\hline 2 & PCIT & $\begin{array}{l}5.18 \mathrm{~m} \\
33.45 \mathrm{f}\end{array}$ & $\begin{array}{c}\text { Adjust } \\
\text { ment }\end{array}$ & Completed & 11 & & & & & 160 & $92 *$ & .07 & .50 \\
\hline 3 & PCIT & $\begin{array}{l}5.97 \mathrm{~m} \\
28.57 \mathrm{~m}\end{array}$ & DBD & Completed & 12 & & & & & 108 & 52 & .50 & .64 \\
\hline 4 & $\mathrm{UC}$ & $\begin{array}{l}6.13 \mathrm{~m} \\
28.32 \mathrm{f}\end{array}$ & ADHD & Continuing & & & 280.75 & 41 & 0 & 216 & 185 & .12 & .41 \\
\hline 5 & PCIT & $\begin{array}{l}5.01 \mathrm{~m}, \\
35.71 \mathrm{f}\end{array}$ & DBD & Continuing & E 17 & & & & & 146 & $123 *$ & .20 & .51 \\
\hline 6 & $\mathrm{UC}$ & $\begin{array}{l}4.67 \mathrm{~m} \\
30.69 \mathrm{f}\end{array}$ & ASD & Completed & & & 245.51 & 93.25 & E 0 & 139 & $129 *$ & .38 & .88 \\
\hline 7 & UC & $\begin{array}{l}3.84 \mathrm{f} \\
22.34 \mathrm{f}\end{array}$ & ASD & Continuing & & & E 384 & E 30 & 0 & 154 & 145 & .14 & .19 \\
\hline 8 & UC & $\begin{array}{l}3.85 \mathrm{~m}, \\
27.12 \mathrm{f}\end{array}$ & ASD & Continuing & & & 116 & 299.75 & 0 & 142 & 142 & .09 & .30 \\
\hline 9 & UC & $\begin{array}{l}5.00 \mathrm{~m} \\
25.30 \mathrm{f}\end{array}$ & DBD & Continuing & & & 114.75 & 43.75 & 0 & 48 & 49 & .26 & .27 \\
\hline 10 & UC & $\begin{array}{l}5.85 \mathrm{~m} \\
27.85 \mathrm{f}\end{array}$ & ADHD & Drop Out & & & 28.5 & 17 & 0 & 177 & 180 & .20 & .54 \\
\hline 11 & $\mathrm{UC}$ & $\begin{array}{l}7.05 \mathrm{~m} \\
31.24 \mathrm{f}\end{array}$ & ADHD & Continuing & & & 227 & 119 & 12 & 168 & 171 & .20 & .20 \\
\hline 12 & UC & $\begin{array}{l}4.29 \mathrm{~m} \\
26.03 \mathrm{f}\end{array}$ & $\begin{array}{l}\text { Asp- } \\
\text { erger }\end{array}$ & Drop Out & & & 34.75 & 15.75 & 0 & 87 & 92 & .27 & .24 \\
\hline 13 & PCIT & $\begin{array}{l}7.21 \mathrm{f} \\
36.16 \mathrm{f}\end{array}$ & $\begin{array}{l}\text { ASD } \\
\text { DBD }\end{array}$ & Continuing & 17 & & & & & 95 & 104 & .21 & .21 \\
\hline 14 & PCIT & $\begin{array}{l}5.36 \mathrm{f} \\
32.85 \mathrm{f}\end{array}$ & $\begin{array}{l}\text { DBD, } \\
\text { Trauma }\end{array}$ & $\begin{array}{l}\text { Drop Out } \\
\text { (CDI) }\end{array}$ & 1 & & & & & 106 & 121 & .04 & .80 \\
\hline 15 & $\mathrm{UC}$ & $\begin{array}{l}5.66 \mathrm{~m} \\
30.04 \mathrm{f}\end{array}$ & ADHD & Continuing & & & E 195 & E 91 & $\begin{array}{l}E \\
26\end{array}$ & 223 & 243 & .52 & .43 \\
\hline 16 & $\mathrm{UC}$ & $\begin{array}{l}4.55 \mathrm{~m} \\
\text { NR f }\end{array}$ & ADHD & $\begin{array}{l}\text { Drop Out } \\
\text { (Home } \\
\text { Hours) }\end{array}$ & & & 28 & 18 & 0 & 148 & 169 & .16 & .07 \\
\hline
\end{tabular}

*Change from Clinically Significant to Normative Range; Bold indicates improvement on both ECBI and Compliance PCIT $=$ Parent-Child Interaction Therapy; $\mathrm{UC}=$ Usual Care; $\mathrm{NR}=$ Not Reported; $\mathrm{m}=$ male; $\mathrm{f}=$ female; age in years; $\mathrm{DBD}=$ Disruptive Behavior Disorder; ASD = Autism Spectrum Disorder; ADHD = Attention Deficit/ Hyperactivity Disorder; Continuing = at the second assessment that family was continuing with same type of therapy as at the first assessment; TSS $=$ Therapeutic Support Staff; BSC $=$ Behavior Specialist Consultant; MT = Mobile Therapist; E = Estimation of dose is based on parent report because therapist report unavailable; Med = child began medication management; Phase in which discontinued PCIT is indicated in parentheses 
Figure 1. Change on ECBI Raw Intensity Scale as Function of Billable Service Hours

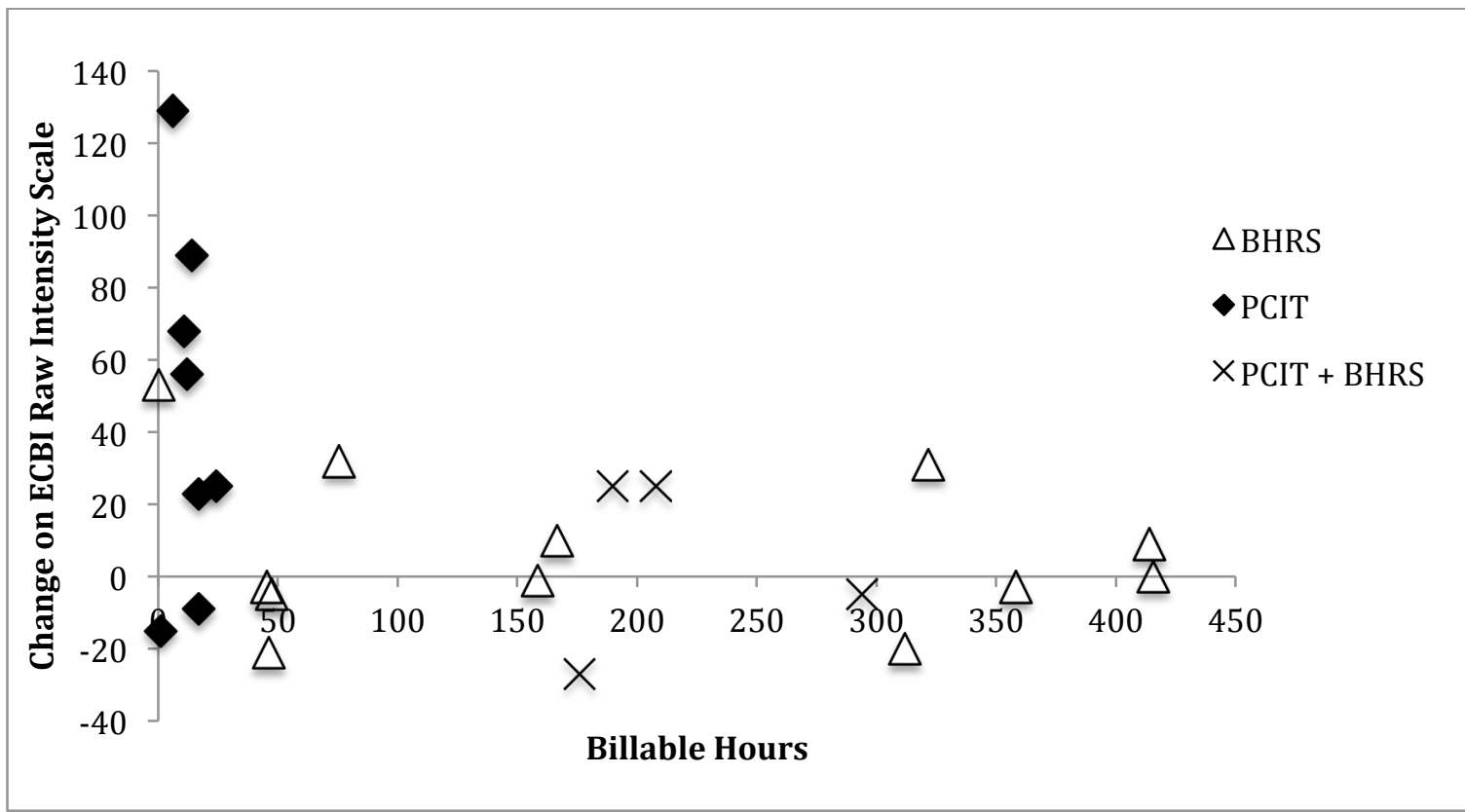

Note: Positive change indicates decreases in disruptive behavior, and negative change indicates increases in disruptive behavior. 
Figure 2. Change on ECBI Raw Intensity Scale as Function of Billable Service Hours for PCIT Group

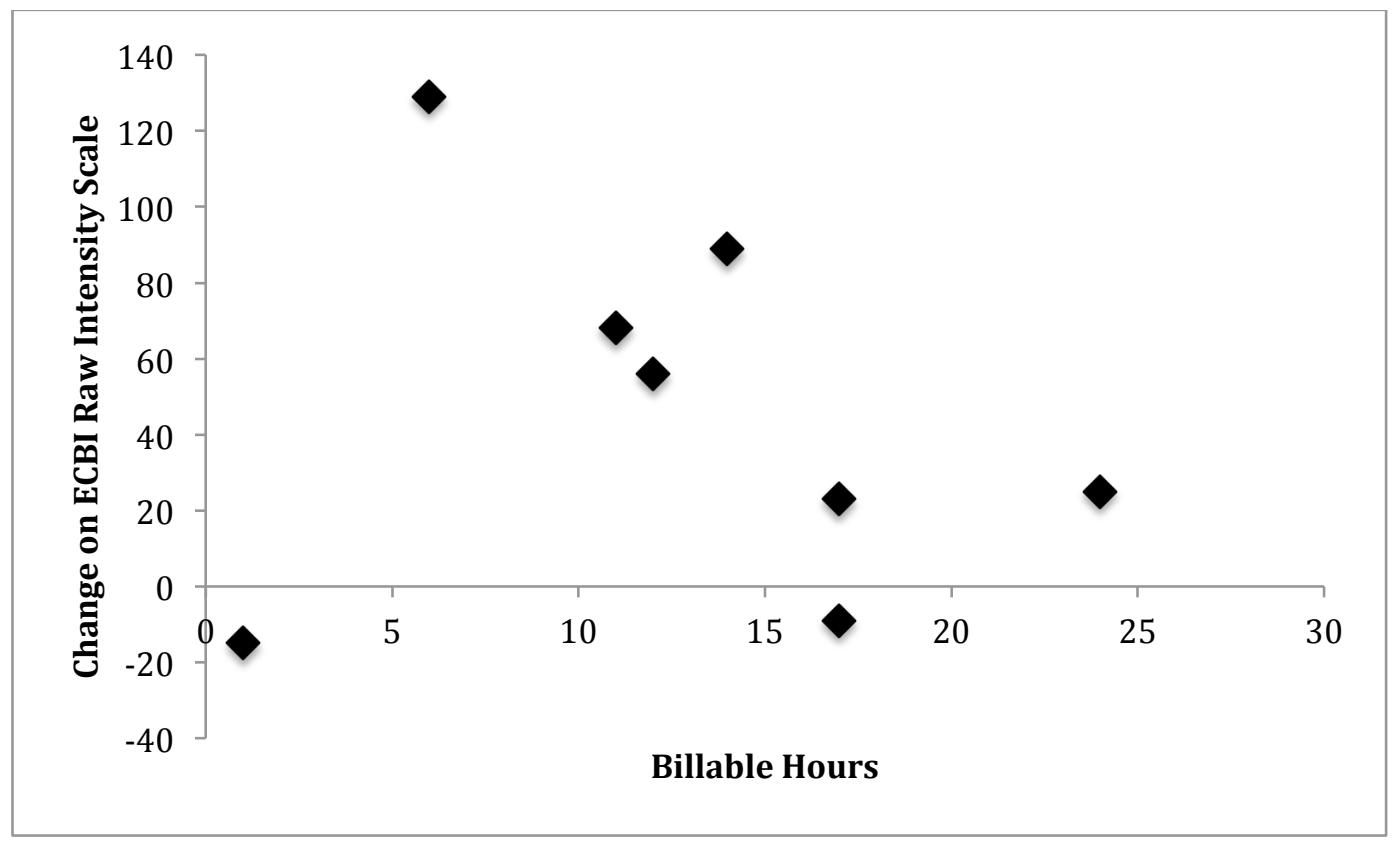

Note: Participants who received BHRS with PCIT were excluded from this graph in order to show detail for the PCIT participants who did not receive BHRS. Positive change indicates improvement in disruptive behavior, and negative change indicates increases in disruptive behavior. 
Figure 3. Effect Sizes from the First to Second Assessment for the PCIT $(N=12)$ and $U C(N=$ 13) Groups Across Objective Outcome Measures

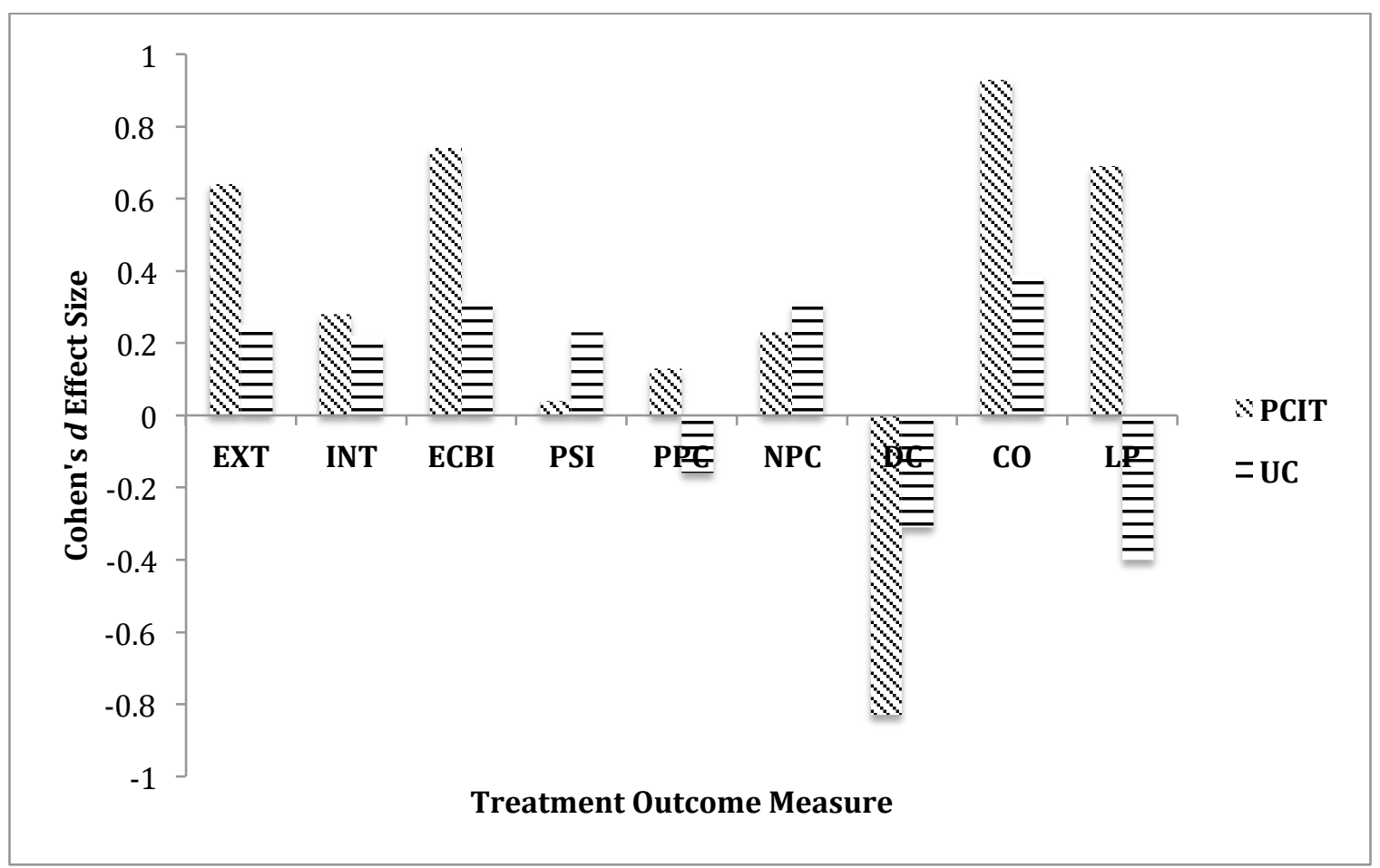

EXT $=$ Child Behavior Checklist Externalizing $T$ Scale, $\mathrm{INT}=$ Child Behavior Checklist Internalizing $T$ Scale, ECBI = Eyberg Child Behavior Inventory raw Intensity Scale, PSI = Parenting Stress Index raw Total Scale, PPC = positive parenting composite, NPC = negative parenting composite, $\mathrm{DC}=$ direct commands divided by total commands, $\mathrm{CO}=$ child compliance, $\mathrm{LP}=$ labeled praise for compliance.

Note: Families in the PCIT group received a median 17 hours per family, of which a median of 10 hours were PCIT sessions. Families in the UC group received a median 113.50 service hours during the study. 
Figure 4. Effect Sizes from the First to Second Assessment for the "pure" PCIT $(N=6)$ and "pure” UC $(N=10)$ Groups Across Objective Outcome Measures

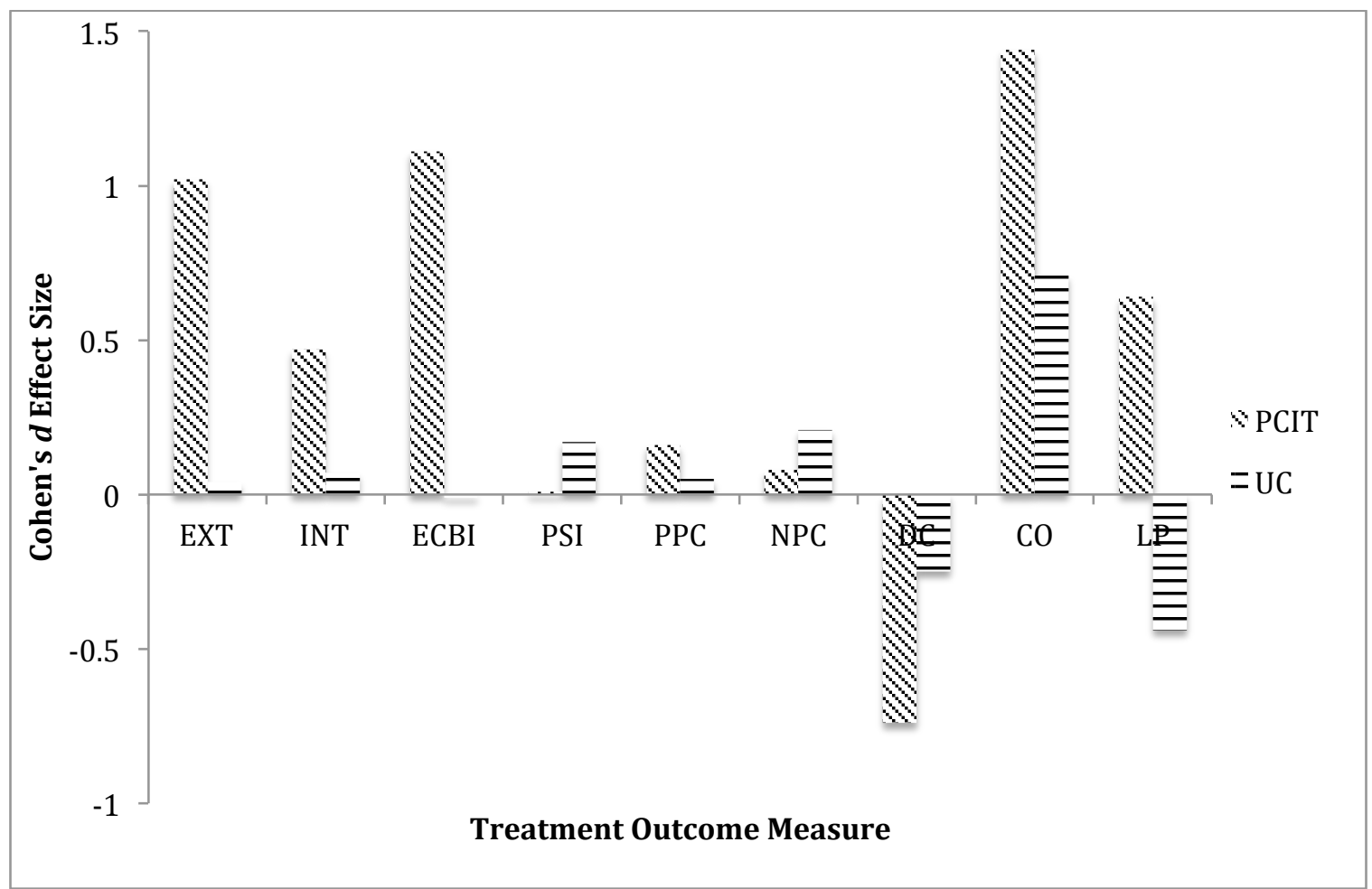

EXT $=$ Child Behavior Checklist Externalizing $T$ Scale, INT $=$ Child Behavior Checklist Internalizing $T$ Scale, ECBI = Eyberg Child Behavior Inventory raw Intensity Scale, PSI = Parenting Stress Index raw Total Scale, $\mathrm{PPC}=$ positive parenting composite, $\mathrm{NPC}=$ negative parenting composite, $\mathrm{DC}=$ direct commands divided by total commands, $\mathrm{CO}=$ child compliance, $\mathrm{LP}=$ labeled praise for compliance.

Note: Families in the PCIT group received a median 12 hours per family. Families in the UC group received a median 338.75 service hours during the study. 
Figure 5. Percentage of Children with Improvement in Each Group

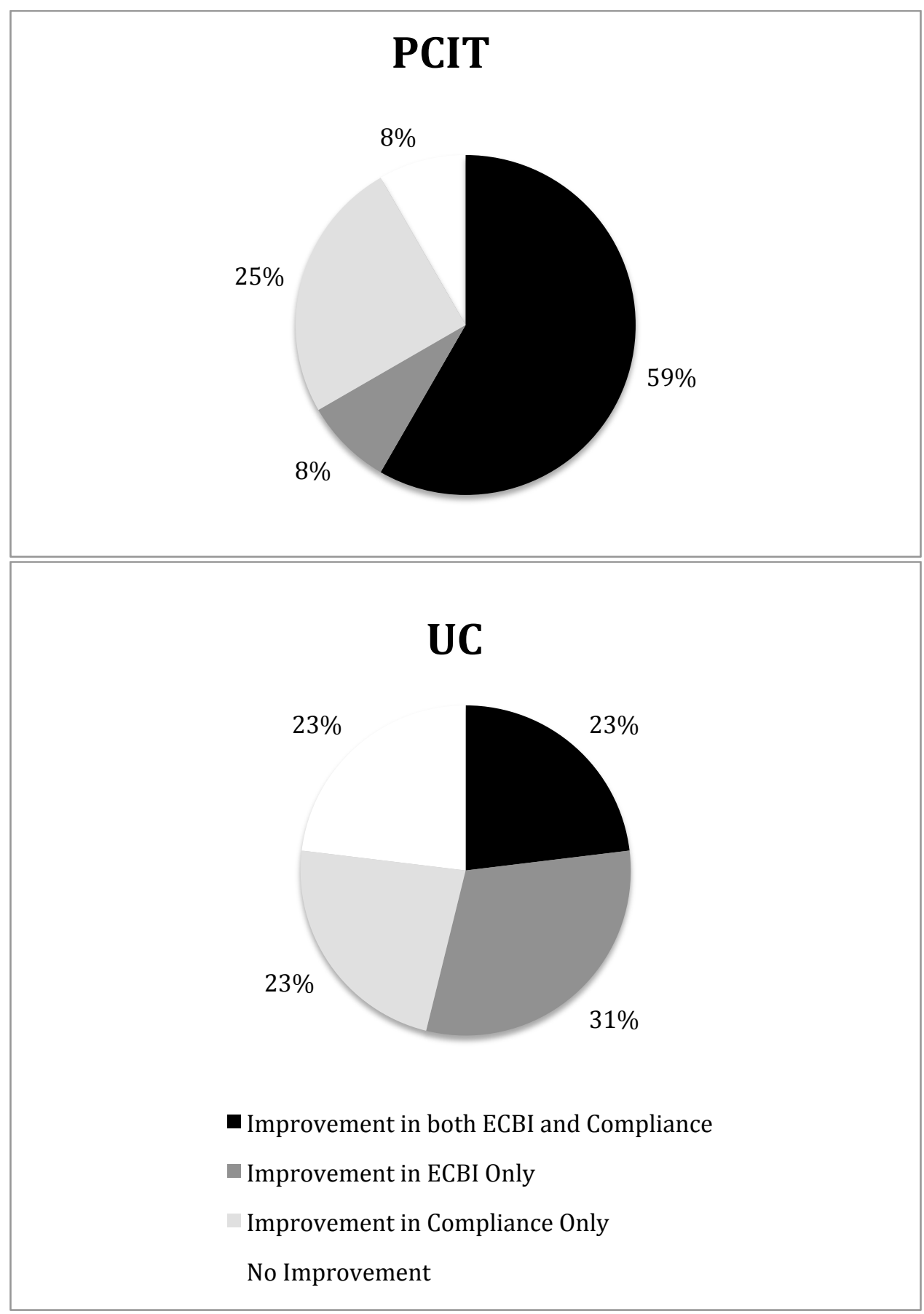

PCIT Group N = 12, UC Group N = 13 
Figure 6. Percentage of Children with Improvement in the "Pure" Groups

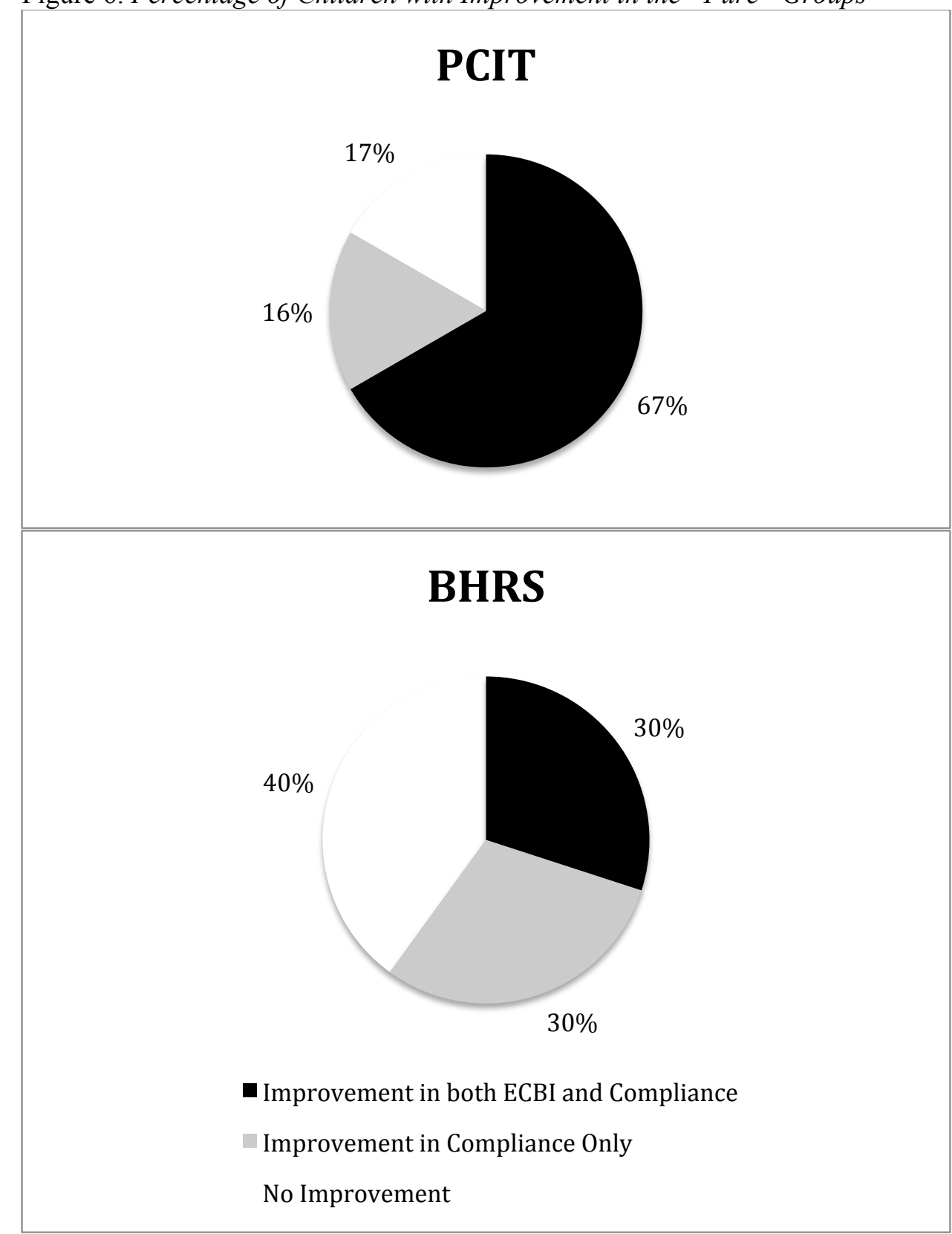

PCIT Group $\mathrm{N}=6$, UC Group $\mathrm{N}=10$ 
Appendix A: Caregiver and Child Information Form 
Caregiver and Child Information Form

Caregiver Name:

Date:

Child Name:

Please answer the following questions about yourself.

What is your sex? Male Female

What is your date of birth?

$1+1+(\mathrm{MM} / \mathrm{DD} / \mathrm{YYYY})$

What is your race?

--African American

--American Indian/ Alaska Native

--Caucasian

--Asian American

--Other (please describe):

Are you Hispanic/ Latino? Yes No

What is your relationship to the participating child?

--Biological parent

--Step- parent

--Foster parent

--Adoptive parent

--Other (please describe):

What is your current marital status?

--Married

--Living with Partner

--Separated

--Divorced

--Never Married

--Other:

What is the highest level of education you have received?

--Less than $9^{\text {th }}$ Grade 
--Some high school

--High school diploma or GED

--Some college

--Associate's degree

--Bachelor's degree

--Some graduate school

--Master's degree

--Doctoral degree

What is your total household income (please write out)?

What is your current employment status?

-- Working full-time

-- Working part-time

-- Not employed but seeking employment

-- Not employed but not seeking employment (e.g., Homemaker)

--Retired

--Other (please describe):

What is your occupation (current or past if unemployed)?

Have you been involved with Child Protective Services/ Child and Youth Services in the past? Yes

No

Are you currently involved with Child Protective Services/ Child and Youth Services?

Yes

No

How many people live in your home?

Please list the people living in your home:

\begin{tabular}{|l|l|l|}
\hline Name & Age & Relationship \\
\hline
\end{tabular}




\begin{tabular}{|l|l|l|}
\hline & & \\
\hline & & \\
\hline & & \\
\hline & & \\
\hline & & \\
\hline & & \\
\hline
\end{tabular}

Have you received counseling/ therapy services before? Yes No

If yes, please describe:

Reason:

When:

Where:

What did the services involve?

Please answer the following questions about your child.

What is your child's sex? Male Female

What is your child's date of birth? 1 (mm/dd/yyyy)

What is your child's race?

Is your child Hispanic/ Latino? Yes No

What medications is your child currently taking?

(Type)

(Dose)

(Frequency) 
Has the participating child received counseling/ therapy services before? Yes No

If yes, please describe:

Reason:

When:

Where:

What did the services involve?

Has your child ever been diagnosed with an Autism Spectrum Disorder? YES

NO 
Appendix B: Therapist Information Form 
Therapist Information Form

Identification Number:

For how many years have you provided clinical services?

What is the highest degree you have obtained? (Please circle.)

Ph. D./ Psy. D./ Ed. D.

M. S. W./ L. S. W.

M. A./ M. S.

M. Ed.

Other: (Please list.)

Describe your theoretical orientation.

What is your sex? (Please circle.)

Male Female

What is your race/ ethnicity? (Please circle.)

Caucasian

African American

Latino/ Hispanic

American Indian

Other: (Please list.)

Have you received training in PCIT from Cheryl B. McNeil or another master trainer?

Yes No 
Appendix C: Clinical Information Form 


\section{Clinical Information Form}

Child:

Please list all billing and other diagnoses for the participating child.

Please list diagnostic impressions for the participating caregiver.

To your knowledge, has this child ever been involved with Child Protective Services/ Child Youth Services?

Yes No 
Appendix D: Therapist Report of Treatment Attendance and Completion 
Therapist Report of Treatment Attendance and Completion

Please fill-out these questions regarding treatment during these dates to

For the child:

1. Did the child met the treatment goal regarding child disruptive behavior (completed treatment for this goal)? YES NO

2. Is the child still receiving treatment services toward meeting the treatment goal regarding child disruptive behavior (in progress)? $\quad$ YES NO

3. Is this child no longer receiving treatment services toward meeting the treatment goal regarding disruptive behavior, has not/ did not meet the treatment goal, and/ or you have not had in-person or telephone contact with the caregiver for the past four weeks (dropped out)? $\quad$ YES NO

4. How many sessions did the child receive toward meeting this treatment goal during the specified dates (Not applicable for BHRS)?

5. What was the approximate number of minutes per session (PCIT)/ total billable hours by type (UC) that the child received toward meeting this treatment goal?

6. How many sessions were cancelled/ did the family not show up during the specified dates (Not applicable for BHRS)? 
Appendix E: PCIT Therapist Follow-Up Survey 


\section{PCIT Therapist Follow-Up Survey}

1. With how many cases in total have you completed PCIT?

2. With how many cases in total have you attempted PCIT or are in the process of providing PCIT?

3. What do you like about PCIT?

4. What do you not like about PCIT?

5. What was most useful about PCIT in helping your clients?

6. What parts of PCIT did you not think were useful in helping your clients?

7. Did you often add any therapeutic practices to your treatment that are not included in PCIT (e.g., token economy, school-home note)? If so, what practices and why? 
8. What aspects of PCIT were difficult to implement and why?

9. Will you continue using PCIT in clinical practice?

10. Would you recommend that a colleague receive training in PCIT?

11. What components of PCIT did you almost always use (e.g., coding, house rules, public behavior, homework)?

12. What componemts of PCIT did you often leave out (e.g., coding, house rules, public behavior, homework)? 
Appendix F: Caregiver Satisfaction Survey 


\section{Caregiver Satisfaction Survey}

Identification \#:

1. What did you like about the services you received for your child?

2. What did you not like about the services you received for your child?

3. What was most useful for helping your child?

4. What did you think was not useful in helping your child?

5. Would you recommend the type of services that you received to another family that was experiencing similar stressors?
(1) DEFINITELY NOT
(2) MAYBE
(3) YES
(4) DEFINITELY YES 
Appendix G: PCIT Treatment Questionnaire 
Therapist's Name

Date of Session

Who was present in the session?

How long did the session last?

What session was this?

Intake

CDI Teach

CDI Coach

PDI Teach

PDI Coach

Termination/Booster

1. Did you give the ECBI before starting the session? YES NO Not Applicable

2. Did you collect homework? YES NO Not Applicable

3. How long did you talk to the parent (not counting coaching time)? minutes

4. How long did you coach the parent with the child? (if 2 caregivers, how long did you coach each?) minutes

5. Did you coach from behind a mirror or in the room? YES NO Not Applicable

6. Did you code the parent using the DPICS? YES NO Not Applicable

7. Did you assign homework? YES NO Not Applicable

8. Did you show the parent their coding data and compare it to past sessions? YES NO NA

9. Did you review the ECBI changes with the parent? YES NO NA

10. How many children were in the room with the caregiver during coaching?

11. Did you spend time alone with the child, without the parent present? YES NO NA

12. Did you look at the PCIT Treatment Manual either immediately before or during the session? Yes NO NA

If this session deviated from usual PCIT, how was it different? 
Appendix H: UC Treatment Questionnaire 


\section{Therapist's Name}

Who is typically present in the session?

Hours of BSC

Hours of TSS

Hours of MT

What name would you give to your intervention (e.g., applied behavior analysis, problem-solving with child, play therapy, parent training, crisis intervention, etc.)?

13. Do you give a standardized parent-report measure of child behavior? YES NO Not Applicable

14. Do you collect homework? YES NO Not Applicable

1. How much time did you spend with the caregiver alone?

2. How much time did you spend with the caregiver and child together? minutes

3. Did you provide in-vivo feedback to the parent on behavior management techniques used during the session? YES NO Not Applicable

4. Do you code child behavior using operationally defined behavior categories? YES NO Not Applicable

5. Do you code parent behavior using operationally defined behavior categories? YES NO Not Applicable

6. Do you assign homework? YES NO Not Applicable

7. Do you show the parent the coded data and compare it to past sessions? YES NO Not Applicable

8. Do you review changes on a standardized parent-report measure of child behavior? YES NO Not Applicable

9. How much time do you spend with child alone? minutes

Describe the techniques you used in your session. 